\title{
WestVirginiaUniversity
}

THE RESEARCH REPOSITORY @ WVU

Graduate Theses, Dissertations, and Problem Reports

2006

\section{Asymptotic solutions of almost diagonal differential and difference systems}

Fei Xue

West Virginia University

Follow this and additional works at: https://researchrepository.wvu.edu/etd

\section{Recommended Citation}

Xue, Fei, "Asymptotic solutions of almost diagonal differential and difference systems" (2006). Graduate Theses, Dissertations, and Problem Reports. 4280.

https://researchrepository.wvu.edu/etd/4280

This Dissertation is protected by copyright and/or related rights. It has been brought to you by the The Research Repository @ WVU with permission from the rights-holder(s). You are free to use this Dissertation in any way that is permitted by the copyright and related rights legislation that applies to your use. For other uses you must obtain permission from the rights-holder(s) directly, unless additional rights are indicated by a Creative Commons license in the record and/ or on the work itself. This Dissertation has been accepted for inclusion in WVU Graduate Theses, Dissertations, and Problem Reports collection by an authorized administrator of The Research Repository @ WVU.

For more information, please contact researchrepository@mail.wvu.edu. 


\title{
Asymptotic Solutions of Almost Diagonal Differential and Difference Systems
}

\author{
Fei Xue \\ Dissertation submitted to the \\ Eberly College of Arts and Sciences \\ at West Virginia University \\ in partial fulfillment of the requirements \\ for the degree of
}

Doctor of Philosophy

in

Mathematics
Harry Gingold, Ph.D., Chair
Ismail B. Celik, Ph.D.
Weifu Fang, Ph.D.
Harumi Hattori, Ph.D.
Dening Li, Ph.D.
Department of Mathematics
West Virginia University
2006

Keywords: asymptotic integration, asymptotic summation, almost diagonal, fundamental solution 


\section{Abstract}

\section{Asymptotic Solutions of Almost Diagonal Differential and Difference Systems}

Fei Xue

New methods for both asymptotic integration of the linear differential systems $Y^{\prime}(t)=$ $[D(t)+R(t)] Y(t)$ and asymptotic summation of the linear difference systems $Y(t+1)=$ $[D(t)+R(t)] Y(t)$ are derived. The fundamental solution $Y(t)=\Phi(t)[I+P(t)]$ for differential and difference systems is constructed in terms of a product. The first matrix function $\Phi(t)$ is decided by the diagonal matrix $D(t)$ and the second matrix $I+P(t)$ is a perturbation of the identity matrix I. Another fundamental solution $Y(t)=[I+Q(t)] \Phi(t)$ is also constructed for difference systems. Conditions are given on the matrix $[D(t)+R(t)]$ that allow us to represent $I+P(t)$ or $Q(t)+I$ as an absolutely convergent resolvent series without imposing stringent conditions on $R(t)$. In particular the analogs, in the setting of difference equations, of fundamental theorems of Levison and Hartman-Wintner are shown to follow from one and same theorem in this work. 


\section{Acknowledgments}

I would like to thank my advisor, Dr. Harry Gingold, for his guidance and help in my study, research and this dissertation. Having such a nice and wise mentor in my life is my honor and luck .

I wold also like to thank other committee members: Dr. Ismail B. Celik, Dr. Weifu Fang, Dr. Harumi Hattori, and Dr. Dening Li for their help during my studies. 


\section{Dedication}

my wife 


\section{Contents}

Chapter 1. Introduction 1

1.1. Almost diagonal differential equations 1

1.2. Almost diagonal difference equations 6

Chapter 2. Left almost diagonal difference equations 9

2.1. Introduction 9

2.2. Some Formal Calculations 10

2.3. A framework for asymptotic approximation 14

2.4. An explicit criteria 22

2.5. Some examples and comparisons 27

Chapter 3. Right almost diagonal differential equations 29

3.1. Introduction 29

3.2. Asymptotic integration 29

3.3. Examples 39

Chapter 4. Right almost diagonal difference equations 46

4.1. Introduction 46

4.2. Asymptotic Summation 46

4.3. Examples $\quad 58$

$\begin{array}{lr}\text { Bibliography } & 68\end{array}$ 


\section{CHAPTER 1}

\section{Introduction}

\subsection{Almost diagonal differential equations}

Consider, the linear matrix differential system

$$
Y^{\prime}(t)=A(t) Y(t)
$$

with $A(t), Y(t)$ be $n \times n$ matrix functions. The basic problem is that the solution vectors $Y(t)$ cannot normally be written as explicit expressions involving the entries of the given matrix $A(t)$. This difficulty brings the interest of asymptotic theory with the goal to approximate the solutions explicitly as $t$ approaches $\infty$. Its importance can hardly be overestimated for more reasons than one. Firstly for its own sake. Secondly for the reason that the asymptotic behavior of solutions of nonlinear problems require quite often asymptotic integration of a linearized problem. Throughout the rest of our work, we assume that (1.1.1) is defined on some interval $[a, \infty)$.

Let $A(t)=D(t)+R(t)$, in which $D(t)$ is the diagonal part of $A(t)$ and $R(t)$ is the off-diagonal part of $A(t)$. Thus we rewrite (1.1.1) as

$$
Y^{\prime}(t)=(D(t)+R(t)) Y(t)
$$

with

$$
\begin{aligned}
& D(t)=\operatorname{diag}\left\{\lambda_{1}(t), \lambda_{2}(t), \ldots, \lambda_{n}(t)\right\}, \\
& R(t)=\left(r_{j k}(t)\right)_{j, k=1}^{n}, \quad \text { where } r_{k k}(t)=0 .
\end{aligned}
$$

Since a fundamental solution of the unperturbed equation $Y^{\prime}(t)=D(t) Y(t)$ is given as

$$
\Phi(t)=\exp \left(\int_{t_{0}}^{t} D(s) d s\right)
$$

where $t_{0} \in[a, \infty)$ is a constant, one may hope that an asymptotic representation of (1.1.2) be given by

$$
Y(t)=(I+Q(t)) \exp \left(\int_{t_{0}}^{t} D(s) d s\right),
$$

with $Q(t) \rightarrow 0$ as $t \rightarrow \infty$. Another option is to look for a solution $Y(t)$ of (1.1.2) that is represented in the form

$$
Y(t)=\exp \left(\int_{t_{0}}^{t} D(s) d s\right)(I+P(t))
$$

with another unknown perturbation $P(t)$ such that $P(t) \rightarrow 0$ as $t \rightarrow \infty$.

The above two asymptotic expressions are called the solutions of "Almost Diagonal Differential Systems" which is defined in $[\mathbf{1 2}, \mathbf{1 3}, \mathbf{1 4}]$. 
Definition 1.1.1. Let $D(t) \in C[a, \infty)$ be a diagonal matrix and let $R(t) \in C[a, \infty)$ be such that its diagonal elements are all zero. We say that the system (1.1.2) is right almost diagonal if it possesses an asymptotic representation (1.1.7) with $P(t) \in C[a, \infty]$ and $P(t) \rightarrow 0$ as $t \rightarrow \infty$. Similarly, if representation (1.1.6) holds, the system (1.1.2) will be called left almost diagonal.

The asymptotic integration of (1.1.2) shall mean in our context the representation of a fundamental solution of (1.1.2) as a left or right almost diagonal system.

We will also study on a special type of differential systems in our work that is called "potentially oscillatory" differential equations. The meaning of "potentially oscillatory" is as follows,

Definition 1.1.2. A differential system (1.1.2) is called potentially oscillatory if

$$
\left|\operatorname{Re} \int_{t_{1}}^{t_{2}}\left(\lambda_{j}(s)-\lambda_{k}(s)\right) d s\right| \leq M
$$

for all $a \leq t_{1}, t_{2} \leq \infty$ where $M$ is a fixed positive number.

The time dependent Schrodinger equation, and in particular the adiabatic approximation theorem in quantum mechanics due to [10], provide an important motivation for our study for potentially oscillatory differential systems. When $A(t)$ is an anti-Hermitian operator, the eigenvalues of $A(t)$ are pure imaginary and of course (1.1.1) is potentially oscillatory. See [1], for an extensive list of references dealing with this important theorem in quantum mechanics. The study of a partially discretized wave equation with a certain varying potential is just one additional instance to which right almost diagonal systems can be beneficial.

An useful proposition for potentially oscillatory differential systems is the following.

Proposition 1.1.3. Assume that (1.1.2) is potentially oscillatory and $\Phi$ is the function defined in (1.1.5), then for any $n \times n$ matrix $C(t)=\left(c_{j k}(t)\right)_{j, k=1}^{n}$,

(i) $\Phi^{-1}(t) C(t) \Phi(t) \rightarrow 0$ as $t \rightarrow \infty$, if $C(t) \rightarrow 0$ as $t \rightarrow \infty$

(ii) $\Phi^{-1}(t) C(t) \Phi(t) \in L^{1}[a, \infty)$, if $C(t) \in L^{1}[a, \infty)$.

Indeed, since $\left|\operatorname{Re} \int_{t_{0}}^{t}\left[\lambda_{k}(s)-\lambda_{j}(s)\right] d s\right| \leq M$, we get $\left|e^{\int_{t_{0}}^{t}\left[\lambda_{k}(s)-\lambda_{j}(s)\right] d s}\right| \leq \tilde{M}$ for some positive constant $\tilde{M}$. Notice that

$$
\left(\Phi^{-1}(t) C(t) \Phi(t)\right)_{j k}=c_{j k}(t) e^{\int_{t_{0}}^{t}\left[\lambda_{k}(s)-\lambda_{j}(s)\right] d s},
$$

and the conclusions (1.1.9) and (1.1.10) follow.

By the Proposition 1.1.3, we are able to point out a relation between left almost diagonal systems and right almost diagonal systems.

THEOREM 1.1.4. Assume the system (1.1.2) is potentially oscillatory. Then, it is left almost diagonal if and only if it is also right almost diagonal.

PROOF. If (1.1.2) is left almost diagonal, it has a fundamental solution

$$
Y(t)=(I+Q(t)) \Phi(t)
$$

in which $\Phi(t)$ is defined in (1.1.5) and $Q(t) \rightarrow 0$ as $t \rightarrow \infty$. We let

$$
P(t)=\Phi^{-1}(t) Q(t) \Phi(t)
$$


and from the property of potentially oscillatory differential systems in (1.1.9), we have that $P(t) \rightarrow 0$ as $t \rightarrow \infty$.

Adding identity matrix $I$ to both sides of (1.1.13) to get

$$
I+P(t)=I+\Phi^{-1}(t) Q(t) \Phi(t)=\Phi^{-1}(t)[I+Q(t)] \Phi(t) .
$$

Multiplying $\Phi(t)$ from the left side to the above equation, we can conclude that

$$
\Phi(t)(I+P(t))=(I+Q(t)) \Phi(t)
$$

which means that the differential system (1.1.2) also has a fundamental solution in the form $\Phi(t)(I+P(t))$ with this $P(t) \rightarrow 0$ as $t \rightarrow \infty$.

We can show that a right almost diagonal system is also left almost diagonal, if it is potentially oscillatory, in a similar way.

The asymptotic representation of (1.1.2) is widely discussed in the literature. Its main goal is to determine conditions on $D(t)$ and $R(t)$ that will guarantee that a fundamental solution of (1.1.2) is left almost diagonal system. (Right almost diagonal systems are more prevalent in the context of quantum mechanics.) An important theorem among them is given by Levinson in 1948 [29].

THEOREM 1.1.5. (Levinson's Theorem) For the differential system (1.1.2), if $R(t) \in$ $L^{1}[a, \infty)$ and $D(t)$ fits the dichotomy conditions: for each pair of integers $j$ and $k$ in $[1, n]$ with $j \neq k$ and for for all $s$ and $t$ such that $a<s<t<\infty$,

$$
\begin{aligned}
& \text { either } \int_{a}^{t} \operatorname{Re}\left(\lambda_{j}(\tau)-\lambda_{k}(\tau)\right) d \tau \rightarrow-\infty \text { and } \int_{s}^{t} \operatorname{Re}\left(\lambda_{j}(\tau)-\lambda_{k}(\tau)\right) d \tau \leq K_{1}, \\
& \text { or } \quad \int_{s}^{t} \operatorname{Re}\left(\lambda_{j}(\tau)-\lambda_{k}(\tau)\right) d \tau \geq K_{2},
\end{aligned}
$$

where $K_{1}$ and $K_{2}$ are some constants, then (1.1.2) is left almost diagonal, i.e., it has solutions with the asymptotic form $Y(t)=(I+Q(t)) \exp \left(\int_{t_{0}}^{t} D(s) d s\right)$ with $Q(t) \rightarrow 0$ as $t \rightarrow \infty$.

One of the reasons that his theorem enjoyed wide acceptance, was that it provided relatively simple yet powerful criteria that would lead to the asymptotic integration of (1.1.2) for potentially oscillatory systems as well as for non-potentially oscillatory systems. So much so that it became the cornerstone of the monograph [9].

Levinson also utilized an extra similarity transformation which rediagonalizes $D(t)+$ $R(t)$. His approach was further enhanced by Harris and Lutz $[\mathbf{2 2}, \mathbf{2 3}]$, who showed how to transform (1.1.2) into a system

$$
Y_{N}^{\prime}=\left(D_{N}(t)+R_{N}(t)\right) Y_{N}
$$

via repeated diagonalizations

$$
Y_{0}=Y, \quad Y_{j-1}=\left(I+Q_{j}\right) Y_{j}, \quad j=1, \cdots, N,
$$

so that $Q_{j}(\infty)=0$ and so that

$$
Y(t)=\prod_{j=1}^{N}\left(I+Q_{j}(t)\right) \exp \left(\int_{t_{0}}^{t} D_{N}(s) d s\right) .
$$


Hartman and Wintner (1955) [24] derived another result about the asymptotic solutions of (1.1.2) which differ from the previous conditions in important respects. Their lengthy original proof was simplified also due to Harris and Lutz.

TheOREM 1.1.6. (The Hartman-Wintner theorem) Let $D(t)$ be an $n \times n$ diagonal matrix as defined in (1.1.3) and let there be a constant $c(>0)$ such that

$$
\left|\operatorname{Re}\left(\lambda_{j}(t)-\lambda_{k}(t)\right)\right| \geq c>0, \quad j \neq k
$$

in some interval $[a, \infty)$. Also, let the $n \times n$ matrix $R(t)$ satisfy

$$
R(t) \in L^{p}
$$

for some $p$ such that $1<p \leq 2$. Then the equation (1.1.2) has the solution in the form (1.1.6).

It became fashionable in the field of asymptotic integration to transform a system (1.1.2) to which Levinson's theorem does not apply, into a new system of differential equations that satisfies the conditions of the Levinson's theorem above. For example, in [32]

THEOREm 1.1.7. (Medina and Pinto) For the differential system

$$
Y^{\prime}(t)=(D(t)+V(t)+R(t)) Y(t)
$$

with $D(t), V(t)$ and $R(t)$ be $n \times n$ continuous matrices for $t \geq a . D(t)=\operatorname{diag}\left\{\lambda_{1}(t), \lambda_{2}(t), \ldots, \lambda_{n}(t)\right\}$ fits the dichotomy conditions of Levinson's theorem in (1.1.15) and (1.1.16). Let $Q(t)=$ $-\int_{t}^{\infty} V(s) d s$ exists for $t \geq a$. Moreover, $V Q, D Q, Q D$, and $R \in L^{1}[a, \infty)$. Then (1.1.22) has a fundamental matrix solution $Y(t)$ such that for $t \rightarrow \infty$

$$
Y(t)=[I+o(1)] \exp \left(\int_{t_{0}}^{t} D(s) d s\right)
$$

U. Elias and H. Gingold, [13], found a new method for asymptotic integration of (1.1.2) that based on the introduction of a certain integral equation that pinpoints sufficient conditions.

THEOREM 1.1.8. (Elias and Gingold) If there exists constants $l_{\alpha \beta} \leq \infty$ such that for all $\alpha, \beta, \gamma, \nu$ with $\alpha \neq \beta, \beta \neq \nu$,

$$
\begin{aligned}
& \int_{l_{\alpha \beta}}^{t} r_{\alpha \beta}\left(t_{1}\right) e^{\int_{t_{1}}^{t}\left(\lambda_{\alpha}-\lambda_{\beta}\right) d s} d t_{1} \rightarrow 0 \\
& \int_{l_{\alpha \gamma}}^{t}\left|\left[\int_{l_{\alpha \beta}}^{t_{1}} r_{\alpha \beta}\left(t_{2}\right) e^{\int_{t_{2}}^{t_{1}}\left(\lambda_{\alpha}-\lambda_{\beta}\right) d s} d t_{2}\right] r_{\beta \nu}\left(t_{1}\right) e^{\int_{t_{1}}^{t}\left(\lambda_{\alpha}-\lambda_{\gamma}\right) d s}\right| d t_{1} \rightarrow 0
\end{aligned}
$$

as $t \rightarrow \infty$, then the equation (1.1.2) has an asymptotic representation (1.1.6) where $Q(t) \rightarrow 0$ as $t \rightarrow \infty$.

This theorem leads to an explicit criteria which will enable us to verify that the assumptions in Theorem 1.1.8 really hold. 
THEOREM 1.1.9. If the diagonal elements in $D(t)$ fit the dichotomy conditions in (1.1.15) and (1.1.16), and for every $\alpha \neq \beta, \nu$

$$
\begin{aligned}
& \frac{r_{\alpha \beta}}{\lambda_{\alpha}-\lambda_{\beta}} \rightarrow 0 \quad \text { as } t \rightarrow \infty, \\
& \left(\frac{r_{\alpha \beta}}{\lambda_{\alpha}-\lambda_{\beta}}\right)^{\prime} \in L^{1}, \\
& r_{\beta \nu}(t) \int_{t}^{\infty}\left|\left(\frac{r_{\alpha \beta}}{\lambda_{\alpha}-\lambda_{\beta}}\right)^{\prime}\right| d t_{1} \in L^{1}
\end{aligned}
$$

and for $(\alpha, \beta)$ such that $\int_{t_{0}}^{t} \operatorname{Re}\left(\lambda_{\alpha}(s)-\lambda_{\beta}(s)\right) d s \rightarrow-\infty$ (i.e., $\left.(\alpha, \beta) \in(1.1 .15)\right)$ also

$$
\begin{aligned}
& r_{\beta \nu}(t) e^{\int_{t_{0}}^{t} \operatorname{Re}\left(\lambda_{\alpha}-\lambda_{\beta}\right) d s} \in L^{1}, \\
& r_{\beta \nu}(t) \int_{t_{0}}^{t}\left(\frac{r_{\alpha \beta}}{\lambda_{\alpha}-\lambda_{\beta}}\right)^{\prime} e^{\int_{t_{2}}^{t} \operatorname{Re}\left(\lambda_{\alpha}-\lambda_{\beta}\right) d s} d t_{2} \in L^{1} \text {, }
\end{aligned}
$$

then the conditions of Theorem 1.1.8 hold.

Elias and Gingold's theorem is the most recent and advanced result about the left almost diagonal linear differential systems. The theorems of Levinson and Hartman-Wintner could be shown to follow from this theorem.

Compare to the large body of literature in left almost diagonal differential systems, the amount of work on right almost diagonal differential systems is relatively small. However, it has been shown in several mathematical physical problems, see, e.g., $[\mathbf{1 5}, \mathbf{1 7}, \mathbf{1 8}]$, that a representation of a fundamental solution in the form $\Phi(I+P)$, where the diagonal matrix $\Phi$ is on the left rather than on the right in the given product, has a merit of its own.

Medina and Pinto, [32], had a result about right almost diagonal systems for the differential systems given in the form (1.1.22).

THEOREM 1.1.10. For the differential system

$$
Y^{\prime}(t)=(D(t)+V(t)+R(t)) Y(t),
$$

let $\Phi$ be a fundamental matrix of $Y^{\prime}=D(t) Y$ such that $Q(t)=-\int_{t}^{\infty} \Phi^{-1}(s) V(s) \Phi(s) d s$ exists for $t \geq a$ and $Q^{\prime} Q, \Phi^{-1} R \Phi$ are integrable functions. Then there exists a fundamental matrix $Y$ such that for $t \rightarrow \infty$ we have

$$
Y(t)=\Phi[I+o(1)]
$$

Elias and Gingold, [12], got a result about right almost diagonal differential systems by using a new method in 2002.

THEOREM 1.1.11. Define $K(t)=\Phi^{-1}(t) R(t) \Phi(t), M_{0}=I$ and $M_{l}(t)=\int_{t}^{\infty} M_{l-1}(s) K(s) d s$ with $l=1,2, \ldots$. If there exists some $m \geq 2$ such that

$$
\begin{aligned}
& M_{l}(t) \rightarrow 0 \text { as } t \rightarrow \infty, l=1,2, \ldots m-1, \\
& M_{m-1}(t) K(t) \in L^{1},
\end{aligned}
$$

then the differential system (1.1.2) is right almost diagonal.

They also obtained an explicit criteria about the potentially oscillatory differential systems. 
THEOREM 1.1.12. If the differential system (1.1.2) is potentially oscillatory, which means that for all $\alpha \neq \beta, \alpha, \beta=1,2, \ldots, n$,

$$
\left|\operatorname{Re} \int_{t_{0}}^{t}\left(\lambda_{\alpha}(s)-\lambda_{\beta}(s)\right) d s\right| \leq C, \quad a \leq t_{0}, t<\infty,
$$

where $C$ is a nonnegative constant, and

$$
\begin{aligned}
& \frac{r_{\alpha \beta}(t)}{\lambda_{\alpha}(t)-\lambda_{\beta}(t)} \rightarrow 0 \quad \text { as } t \rightarrow \infty, \\
& \left(\frac{r_{\alpha \beta}(t)}{\lambda_{\alpha}(t)-\lambda_{\beta}(t)}\right)^{\prime} \in L^{1}[a, \infty),
\end{aligned}
$$

and for all $k \neq \beta$,

$$
\left(\int_{t}^{\infty}\left|\left(\frac{r_{\alpha \beta}\left(t_{1}\right)}{\lambda_{\alpha}\left(t_{1}\right)-\lambda_{\beta}\left(t_{1}\right)}\right)^{\prime}\right| d t_{1}\right)\left|r_{\beta k}(t)\right| \in L^{1}[a, \infty) .
$$

Then a fundamental solution of (1.1.2) is given by $Y=\Phi(t)[I+P(t)]$ with $P(t) \rightarrow 0$ as $t \rightarrow \infty$.

\subsection{Almost diagonal difference equations}

Consider the difference system, for $t \geq a$

$$
Y(t+1)=(D(t)+R(t)) Y(t)
$$

where

$$
\begin{aligned}
& D(t)=\operatorname{diag}\left\{\lambda_{1}(t), \lambda_{2}(t), \ldots, \lambda_{n}(t)\right\}, \\
& R(t)=\left(r_{j k}(t)\right)_{j, k=1}^{n}, \quad \text { with } \quad r_{k k}(t)=0 .
\end{aligned}
$$

Similar to the case in the differential systems, since a fundamental solution of the unperturbed equation $Y(t+1)=D(t) Y(t)$ is

$$
\Phi(t)=\prod_{l=a}^{t-1} D(l)
$$

we want to seek an asymptotic representation of a fundamental solution of the perturbed system (1.2.1) be given by

$$
Y(t)=[I+Q(t)] \prod_{l=a}^{t-1} D(l)
$$

with $Q(t) \rightarrow 0$ as $t \rightarrow \infty$. Or, an alternative form

$$
Y(t)=\left(\prod_{l=a}^{t-1} D(l)\right)[I+P(t)]
$$

with another unknown perturbation $P(t)$ such that $P(t) \rightarrow 0$ as $t \rightarrow \infty$. 
Definition 1.2.1. Let $D(t)$ be a diagonal matrix and let $R(t)$ be such that its diagonal elements are all zero. We say that the difference system (1.2.1) is right almost diagonal if it possesses an asymptotic representation (1.2.6) with $P(t) \rightarrow 0$ as $t \rightarrow \infty$. Similarly, if representation (1.2.5) holds and $Q(t) \rightarrow 0$ as $t \rightarrow \infty$, the system (1.2.1) will be called left almost diagonal.

The asymptotic summation of (1.2.1) shall mean in our context the representation of a fundamental solution of (1.2.1) as a left or right almost diagonal system.

We also have the definition of potentially oscillatory difference equations.

Definition 1.2.2. The difference system (1.2.1) is potentially oscillatory on $[a, \infty)$ if there exists some positive number $M_{1}$ and $M_{2}$ such that

$$
0<M_{2} \leq\left|\prod_{l=t_{1}}^{t_{2}} \frac{\lambda_{k}(l)}{\lambda_{j}(l)}\right| \leq M_{1}, \quad j, k=1,2, \cdots n, \quad j \neq k,
$$

for all $a \leq t_{1}<t_{2} \leq \infty$.

Similar to differential equations, we can obtain an useful proposition of potentially oscillatory difference systems.

Proposition 1.2.3. Assume that (1.2.1) is potentially oscillatory, then for any $n \times n$ matrix $G(t):=\left(g_{j k}(t)\right)_{j, k=1}^{n}$, we have

$$
\begin{aligned}
& (\text { i }) \quad \Phi^{-1}(t) G(t) \Phi(t) \rightarrow 0 \text { as } t \rightarrow \infty, \text { if } G(t) \rightarrow 0 \text { as } t \rightarrow \infty, \\
& (\text { ii }) \Phi^{-1}(t) G(t) \Phi(t) \in l^{1}[a, \infty), \text { if } G(t) \in l^{1}[a, \infty) .
\end{aligned}
$$

This time, notice that

$$
\left(\Phi^{-1}(t) G(t) \Phi(t)\right)_{j k}=g_{j k}(t) \prod_{t_{1}=a}^{t-1} \frac{\lambda_{k}\left(t_{1}\right)}{\lambda_{j}\left(t_{1}\right)},
$$

and then the conclusions (1.2.8) and (1.2.9) are easily verified by (1.2.7).

There exists a large body of literature on the asymptotic integration of systems of differential equations. In contrast the amount of works on asymptotic summation of difference systems is relatively small.

Benzaid and Lutz, [3], set up a theorem which is the analog, in the setting of difference equations, to Levinson's theorem using the dichotomy conditions in the setting of differential systems.

THEOREM 1.2.4. (Benzaid and Lutz) For the difference system (1.2.1) with $D(t)$ and $R(t)$ defined in (1.2.2) and (1.2.3), assume on $[a, \infty)$

(1) we have

$$
\lambda_{j}(t) \neq 0 \quad \text { for all } 1 \leq j \leq n \text { and } t \geq a
$$

(2) $R(t)$ satisfies

$$
\left|\frac{1}{\lambda_{j}(t)}\right|\|R(t)\| \in l^{1}[a, \infty), \quad \text { for all } j=1,2 \ldots, n ;
$$


(3) $D(t)$ satisfies the following "Dichotomy Conditions": for each pair of integers $j \neq k$ and for all $t_{1}$ and $t_{2}$ such that $a \leq t_{1} \leq t_{2}<\infty$, either

$$
\prod_{l=a}^{t}\left|\frac{\lambda_{j}(l)}{\lambda_{k}(l)}\right| \rightarrow 0 \text { as } t \rightarrow \infty, \quad \text { and } \prod_{l=t_{1}}^{t_{2}}\left|\frac{\lambda_{j}(l)}{\lambda_{k}(l)}\right| \leq K_{1}
$$

or

$$
\prod_{l=t_{1}}^{t_{2}}\left|\frac{\lambda_{j}(l)}{\lambda_{k}(l)}\right| \geq K_{2}
$$

where $K_{1}$ and $K_{2}$ are some constants.

Then the difference system (1.2.1) has a fundamental matrix satisfying, as $t \rightarrow \infty$,

$$
Y(t)=[I+o(1)] \prod_{l=a}^{t-1} D(l)
$$

S. Bodine and D. Lutz, [4] recently got a result when the perturbation $R(t)$ belongs to $l^{p}$ with $1<p \leq 2$.

THEOREM 1.2.5. For the system $(1.2 .1)$ on $[a, \infty)$, assume that

$$
\left|\lambda_{j}(t)\right| \geq \delta>0 \quad \text { for } 1 \leq j \leq n .
$$

Assume that $D(t)$ satisfies the following conditions: There exist constants $K>0$ and $q \in$ $(0,1)$ such that for each index pair $(j, k), j \neq k$,

$$
\begin{aligned}
& \text { either } \prod_{l=t_{1}}^{t_{2}-1}\left|\frac{\lambda_{k}(l)}{\lambda_{j}(l)}\right| \leq K q^{t_{2}-t_{1}}, \quad \text { for } \forall a \leq t_{1} \leq t_{2}, \\
& \text { or } \prod_{l=t_{1}}^{t_{2}-1}\left|\frac{\lambda_{j}(l)}{\lambda_{k}(l)}\right| \leq K q^{t_{2}-t_{1}}, \quad \text { for } \forall a \leq t_{1} \leq t_{2} .
\end{aligned}
$$

Let $\|R(t)\| \in l^{p}[a, \infty)$ for some $1<p \leq 2$. Then the system (1.2.1) has a fundamental solution $Y(t)=[I+o(1)] \prod_{l=a}^{t-1} D(l)$. 


\section{CHAPTER 2}

\section{Left almost diagonal difference equations}

\subsection{Introduction}

In this chapter, we consider the matrix difference equation

$$
Y(t+1)=A(t) Y(t), \quad t \geq a
$$

with $A(t), Y(t)$ be $n \times n$ matrix functions. We let $A(t)=D(t)+R(t)$ such that $D(t)$ is the diagonal part of $A(t)$ and $R(t)$ is the off-diagonal part. Throughout this chapter we adopt the following notation,

$$
Y(t+1)=[D(t)+R(t)] Y(t)
$$

with

$$
D(t)=\operatorname{diag}\left\{\lambda_{1}(t), \cdots, \lambda_{n}(t)\right\}, \quad R(t)=\left(r_{j k}(t)\right)_{j, k=1}^{n} .
$$

Also,

$$
\Phi(t)=\prod_{l=a}^{t-1} D(l),
$$

is the fundamental solution of the unperturbed equation $Y(t+1)=D(t) Y(t)$.

We will derive some new settings of sufficient conditions under which (2.1.2) is left almost diagonal, i.e., (2.1.2) has a fundamental solution given in the form

$$
Y(t)=[I+Q(t)] \prod_{l=a}^{t-1} D(l)
$$

with $Q(t) \rightarrow 0$ as $t \rightarrow \infty$.

The order of events in this chapter is as follows. In Section 2.2 we derive a summation equation for the perturbation matrix $Q(t)$. In Section 2.3 we formulate Theorem 2.3.1 that is the point of departure from which new and old theorems follow. We also show in Section 2.3 how the analog of Levinson's theorem as well as the analog of Hartman-Wintner theorem in the setting of difference equations follow from one and same formulas in our Theorem 2.3.1 albeit by using different estimates. In Section 2.4 we apply summation by parts that bring out an explicit criteria and show the important fact that quotients of the type $\frac{r_{\alpha \beta}(t)}{\lambda_{\beta}(t)-\lambda_{\alpha}(t)}$ play an important role in asymptotic summation. Section 2.5 is devoted to examples and comparisons. 


\subsection{Some Formal Calculations}

Let $\Phi(t)$ be the solution (2.1.4) of the unperturbed equation $Y(t+1)=D(t) Y(t)$, i.e., $\Phi(t+1)=D(t) \Phi(t)$. With this $\Phi(t)$ we put $Y(t)=\Phi(t)[I+P(t)]$ into (2.1.2) to get

$$
\Phi(t+1)[I+P(t+1)]=[D(t)+R(t)] \Phi(t)[I+P(t)]
$$

i.e.,

$$
\Delta P(t)=\Phi^{-1}(t+1) R(t) \Phi(t)+\Phi^{-1}(t+1) R(t) \Phi(t) P(t)
$$

Let

$$
\begin{aligned}
& \hat{R}(t)=D^{-1}(t) R(t) \\
& K(t)=\Phi^{-1}(t) \hat{R}(t) \Phi(t)=\left(\prod_{l=a}^{t-1} D^{-1}(l)\right) \hat{R}(t)\left(\prod_{l=a}^{t-1} D(l)\right)
\end{aligned}
$$

Here

$$
\hat{R}(t)=\left(\frac{r_{j k}(t)}{\lambda_{j}(t)}\right)_{j, k=1}^{n}, \quad K(t)=\left(\widehat{r}_{j k}(t) \prod_{l=a}^{t-1} \frac{\lambda_{k}(l)}{\lambda_{j}(l)}\right)_{j, k=1}^{n} .
$$

From now on $\prod_{l=a}^{t-1} \frac{\lambda_{j}(l)}{\lambda_{i}(l)}$ will be written in an abbreviated form $\prod_{a}^{t-1} \frac{\lambda_{j}}{\lambda_{i}}$.

Since $\Phi(t+1)=D(t) \Phi(t)$, we can obtain $\Phi^{-1}(t+1)=\Phi^{-1}(t) D(t)$. By the notation (2.2.2) and (2.2.3), $\Phi^{-1}(t+1) R(t) \Phi(t)$ could be written as

$$
\Phi^{-1}(t+1) R(t) \Phi(t)=\Phi^{-1}(t) D(t) R(t) \Phi(t)=\Phi^{-1}(t) \hat{R}(t) \Phi(t)=K(t)
$$

Thus, Eq. (2.2.1) becomes

$$
\Delta P(t)=K(t)+K(t) P(t)
$$

or, component-wise,

$$
\Delta p_{j k}(t)=\widehat{r}_{j k}(t) \prod_{a}^{t-1} \frac{\lambda_{k}}{\lambda_{j}}+\sum_{h=1}^{n}\left(\left(\widehat{r}_{j h}(t) \prod_{a}^{t-1} \frac{\lambda_{h}}{\lambda_{j}}\right) p_{h k}(t)\right), \quad j, k=1, \cdots n .
$$

Instead of this difference equation, we consider a summed version. The limits of summation of individual terms may be different, so at present we denote them formally as $l_{j k}$. The exact value of each $l_{j k}$ will be determined in the sequel. Since the sum (anti-difference) of a function has two forms that depend on the limit, we define

$$
\bigoplus_{t_{1}=l}^{t} f\left(t_{1}\right)= \begin{cases}-\sum_{t_{1}=t}^{l} f\left(t_{1}\right), & \text { if } \quad l \geq t \\ \sum_{t_{1}=l}^{t-1} f\left(t_{1}\right), & \text { if } \quad l<t\end{cases}
$$

and accordingly,

$$
p_{j k}(t)=\bigoplus_{t_{1}=l_{j k}}^{t} \widehat{r}_{j k}\left(t_{1}\right) \prod_{a}^{t_{1}-1} \frac{\lambda_{k}}{\lambda_{j}}+\bigoplus_{t_{1}=l_{j k}}^{t} \sum_{h=1}^{n}\left(\left(\widehat{r}_{j h}\left(t_{1}\right) \prod_{a}^{t_{1}-1} \frac{\lambda_{h}}{\lambda_{j}}\right) p_{h k}\left(t_{1}\right)\right)
$$


The summed version obviously implies the original difference equation (2.2.6). Formally (2.2.8) will be written as

$$
P(t)=\bigoplus_{t_{1}=L}^{t} K\left(t_{1}\right)+\bigoplus_{t_{1}=L}^{t} K\left(t_{1}\right) P\left(t_{1}\right)
$$

By Abel's summation formula, we can easily get a version of the summation by parts method for the operator $\bigoplus$ : for any matrix function $A(t)$ and $B(t)$, we have that in their domain,

$$
\bigoplus_{t_{1}=L}^{t} A\left(t_{1}\right) B\left(t_{1}\right)=\left(\bigoplus_{t_{1}=L}^{t} A\left(t_{1}\right)\right) B(t)-\bigoplus_{t_{1}=L}^{t}\left(\bigoplus_{t_{2}=L}^{t_{1}+1} A\left(t_{2}\right)\right) \triangle B\left(t_{1}\right)
$$

We then use summation by parts on the last term of (2.2.9) to get

$$
\begin{aligned}
P(t) & =\bigoplus_{t_{1}=L}^{t} K\left(t_{1}\right)+\left(\bigoplus_{t_{1}=L}^{t} K\left(t_{1}\right)\right) P(t)-\bigoplus_{t_{1}=L}^{t}\left(\bigoplus_{t_{2}=L}^{t_{1}+1} K\left(t_{2}\right)\right) \triangle P\left(t_{1}\right) \\
& =\bigoplus_{t_{1}=L}^{t} K\left(t_{1}\right)+\left(\bigoplus_{t_{1}=L}^{t} K\left(t_{1}\right)\right) P(t)-\bigoplus_{t_{1}=L}^{t}\left(\bigoplus_{t_{2}=L}^{t_{1}+1} K\left(t_{2}\right)\right)\left[K\left(t_{1}\right)+K\left(t_{1}\right) P\left(t_{1}\right)\right] .
\end{aligned}
$$

Denote

$$
\begin{aligned}
& M_{1}(t)=\bigoplus_{t_{1}=L}^{t} K\left(t_{1}\right) \\
& M_{2}(t)=\bigoplus_{t_{1}=L}^{t} M_{1}\left(t_{1}+1\right) K\left(t_{1}\right)=\bigoplus_{t_{1}=L}^{t}\left(\bigoplus_{t_{2}=L}^{t_{1}+1} K\left(t_{2}\right)\right) K\left(t_{1}\right) .
\end{aligned}
$$

With this notation (2.2.11) may be rewritten as

$$
\left[I-M_{1}(t)\right] P(t)=M_{1}(t)-M_{2}(t)-\bigoplus_{t_{1}=L}^{t} M_{1}\left(t_{1}+1\right) K\left(t_{1}\right) P\left(t_{1}\right) .
$$

We now introduce a matrix $Q(t)=\left(q_{j k}(t)\right)$ related to $P(t)$ via

$$
\Phi(I+P)=(I+Q) \Phi
$$

and proceed to find an asymptotic representation of the form $Y=(I+Q) \Phi$ rather than $Y=\Phi(I+P)$. The relation (2.2.13) is equivalent to

$$
P(t)=\Phi^{-1}(t) Q(t) \Phi(t)
$$

After the substitution of (2.2.14) into Eq. (2.2.12), it becomes

$$
\begin{aligned}
& {\left[I-M_{1}(t)\right] \Phi^{-1}(t) Q(t) \Phi(t) } \\
= & M_{1}(t)-M_{2}(t)-\bigoplus_{t_{1}=L}^{t} M_{1}\left(t_{1}+1\right) K\left(t_{1}\right) \Phi^{-1}\left(t_{1}\right) Q\left(t_{1}\right) \Phi\left(t_{1}\right) .
\end{aligned}
$$


Multiplication by $\Phi(t)$ from the left-hand side and by $\Phi^{-1}(t)$ from the right-hand side leads to

$$
\begin{aligned}
{\left[I-\Phi(t) M_{1}(t) \Phi^{-1}(t)\right] Q(t)=} & \Phi(t) M_{1}(t) \Phi^{-1}(t)-\Phi(t) M_{2}(t) \Phi^{-1}(t) \\
& -\bigoplus_{t_{1}=L}^{t} \Phi(t)\left[M_{1}\left(t_{1}+1\right) K\left(t_{1}\right) \Phi^{-1}\left(t_{1}\right) Q\left(t_{1}\right) \Phi\left(t_{1}\right)\right] \Phi^{-1}(t) .
\end{aligned}
$$

This is our basic equation for the unknown $Q(t)$. Note that a direct substitution of $Y=$ $(I+Q) \Phi$ into the difference equation (2.1.2) leads to a difference equation

$$
Q(t+1)=[D(t)+R(t)] Q(t) D^{-1}(t)+R(t) D^{-1}(t)
$$

which is far less convenient than the difference equation (2.2.5). Consequently, the derivation of the equation (2.2.16) for $Q(t)$ is simpler.

Consider the term $\Phi(t) M_{1}(t) \Phi^{-1}(t)$ which appears on both sides of (2.2.16).

$$
\begin{aligned}
\Phi(t) M_{1}(t) \Phi^{-1}(t) & =\Phi(t)\left(\bigoplus_{t_{1}=L}^{t} K\left(t_{1}\right)\right) \Phi^{-1}(t) \\
& =\Phi(t)\left(\bigoplus_{t_{1}=L}^{t} \Phi^{-1}\left(t_{1}\right) \hat{R}\left(t_{1}\right) \Phi\left(t_{1}\right)\right) \Phi^{-1}(t) \\
& =\bigoplus_{t_{1}=L}^{t} \Phi(t) \Phi^{-1}\left(t_{1}\right) \hat{R}\left(t_{1}\right) \Phi\left(t_{1}\right) \Phi^{-1}(t) .
\end{aligned}
$$

The term $\Phi(t) \Phi^{-1}\left(t_{1}\right)$ in (2.2.17) is diagonal and a typical diagonal entry of $\Phi(t) \Phi^{-1}\left(t_{1}\right)$ is

$$
\left(\Phi(t) \Phi^{-1}\left(t_{1}\right)\right)_{\alpha \alpha}=\prod_{l=a}^{t-1} \lambda_{\alpha}(l) \prod_{k=a}^{t_{1}-1} \frac{1}{\lambda_{\alpha}(k)}= \begin{cases}\prod_{l=t_{1}}^{t-1} \lambda_{\alpha}(l) & \text { if } t_{1}<t \\ \prod_{l=t}^{t_{1}-1} \frac{1}{\lambda_{\alpha}(l)} & \text { if } t_{1}>t \\ 1 & \text { if } t_{1}=t\end{cases}
$$

We define for any function $f(t)$

$$
\coprod_{l=t_{1}}^{t} f(l)= \begin{cases}\prod_{l=t_{1}}^{t-1} f(l) & \text { if } t_{1}<t \\ \prod_{l=t}^{t_{1}-1} \frac{1}{f(l)} & \text { if } t_{1}>t \\ 1 & \text { if } t_{1}=t\end{cases}
$$

then (2.2.18) can be written as

$$
\left(\Phi(t) \Phi^{-1}\left(t_{1}\right)\right)_{\alpha \alpha}=\coprod_{l=t_{1}}^{t} \lambda_{\alpha}(l)
$$

Similarly, a typical diagonal entry of $\Phi\left(t_{1}\right) \Phi^{-1}(t)$, say $(\beta, \beta)$ is

$$
\left(\Phi\left(t_{1}\right) \Phi^{-1}(t)\right)_{\beta \beta}=\coprod_{l=t_{1}}^{t} \frac{1}{\lambda_{\beta}(l)} .
$$

Therefore, a typical entry of $\Phi(t) M_{1}(t) \Phi^{-1}(t)$ is

$$
\left(\Phi(t) M_{1}(t) \Phi^{-1}(t)\right)_{\alpha \beta}=\bigoplus_{t_{1}=l_{\alpha \beta}}^{t} \widehat{r}_{\alpha \beta}\left(t_{1}\right) \coprod_{t_{1}}^{t} \frac{\lambda_{\alpha}}{\lambda_{\beta}} .
$$


From now on, we also write $\coprod_{l=t_{1}}^{t} \frac{\lambda_{\alpha}(l)}{\lambda_{\beta}(l)}$ in an abbreviated form $\coprod_{t_{1}}^{t} \frac{\lambda_{\alpha}}{\lambda_{\beta}}$. Next we return to the last term in $(2.2 .16)$,

$$
\bigoplus_{t_{1}=L}^{t} \Phi(t)\left[M_{1}\left(t_{1}+1\right) K\left(t_{1}\right) \Phi^{-1}\left(t_{1}\right) Q\left(t_{1}\right) \Phi\left(t_{1}\right)\right] \Phi^{-1}(t) .
$$

By substitution of the proper expressions for $M_{1}\left(t_{1}+1\right)$ and $K\left(t_{1}\right)$ and reorganizing the functions $\Phi, \Phi^{-1}$, it becomes

$$
\begin{gathered}
\bigoplus_{t_{1}=L}^{t} \Phi(t)\left(\bigoplus_{t_{2}=L}^{t_{1}+1} \Phi^{-1}\left(t_{2}\right) \hat{R}\left(t_{2}\right) \Phi\left(t_{2}\right)\right)\left(\Phi^{-1}\left(t_{1}\right) \hat{R}\left(t_{1}\right) \Phi\left(t_{1}\right)\right) \Phi^{-1}\left(t_{1}\right) Q\left(t_{1}\right) \Phi\left(t_{1}\right) \Phi^{-1}(t) \\
(2.2 .24)=\bigoplus_{t_{1}=L}^{t} \Phi(t) \Phi^{-1}\left(t_{1}\right)\left[\left(\bigoplus_{t_{2}=L}^{t_{1}+1} \Phi\left(t_{1}\right) \Phi^{-1}\left(t_{2}\right) \hat{R}\left(t_{2}\right) \Phi\left(t_{2}\right) \Phi^{-1}\left(t_{1}\right)\right) \hat{R}\left(t_{1}\right) Q\left(t_{1}\right)\right] \Phi\left(t_{1}\right) \Phi^{-1}(t) .
\end{gathered}
$$

The internal summation is similar to (2.2.17) whose elements had been calculated in (2.2.22). Consequently the $(\alpha, \gamma)$ term of $(2.2 .24)$ is

$$
\bigoplus_{t_{1}=l_{\alpha \gamma}}^{t} \sum_{\beta=1}^{n}\left[\bigoplus_{t_{2}=l_{\alpha \beta}}^{t_{1}+1} \widehat{r}_{\alpha \beta}\left(t_{2}\right) \coprod_{t_{2}}^{t_{1}} \frac{\lambda_{\alpha}}{\lambda_{\beta}}\right]\left[\hat{R}\left(t_{1}\right) Q\left(t_{1}\right)\right]_{\beta \gamma} \coprod_{t_{1}}^{t} \frac{\lambda_{\alpha}}{\lambda_{\gamma}} .
$$

A typical term $(\beta, \gamma)$ of $\hat{R}\left(t_{1}\right) Q\left(t_{1}\right)$ is $\sum_{\nu=1}^{n} \widehat{r}_{\beta \nu}\left(t_{1}\right) q_{\nu \gamma}\left(t_{1}\right)$. We substitute it in (2.2.25) and obtain

$$
\bigoplus_{t_{1}=l_{\alpha \gamma}}^{t} \sum_{\nu=1}^{n} \sum_{\beta=1}^{n}\left(\left[\bigoplus_{t_{2}=l_{\alpha \beta}}^{t_{1}+1} \widehat{r}_{\alpha \beta}\left(t_{2}\right) \coprod_{t_{2}}^{t_{1}} \frac{\lambda_{\alpha}}{\lambda_{\beta}}\right] \widehat{r}_{\beta \nu}\left(t_{1}\right) \coprod_{t_{1}}^{t} \frac{\lambda_{\alpha}}{\lambda_{\gamma}}\right) q_{\nu \gamma}\left(t_{1}\right) .
$$

Consider an individual term in the double summation (2.2.26) that contains the sole element $q_{\nu \gamma}\left(t_{1}\right)$ of the matrix $Q\left(t_{1}\right)$. It is given by

$$
\bigoplus_{t_{1}=l_{\alpha \gamma}}^{t} \sum_{\beta=1}^{n}\left(\left[\bigoplus_{t_{2}=l_{\alpha \beta}}^{t_{1}+1} \widehat{r}_{\alpha \beta}\left(t_{2}\right) \coprod_{t_{2}}^{t_{1}} \frac{\lambda_{\alpha}}{\lambda_{\beta}}\right] \widehat{r}_{\beta \nu}\left(t_{1}\right) \coprod_{t_{1}}^{t} \frac{\lambda_{\alpha}}{\lambda_{\gamma}}\right) q_{\nu \gamma}\left(t_{1}\right) .
$$

The term $\Phi(t) M_{2}(t) \Phi^{-1}(t)$ on the right-hand side of (2.2.16) is calculated similarly.

$$
\begin{aligned}
& \Phi(t) M_{2}(t) \Phi^{-1}(t) \\
= & \Phi(t)\left[\bigoplus_{t_{1}=L}^{t}\left(\bigoplus_{t_{2}=L}^{t_{1}+1} \Phi^{-1}\left(t_{2}\right) \hat{R}\left(t_{2}\right) \Phi\left(t_{2}\right)\right)\left(\Phi^{-1}\left(t_{1}\right) \hat{R}\left(t_{1}\right) \Phi\left(t_{1}\right)\right)\right] \Phi^{-1}(t) \\
(2.2 .28)= & \bigoplus_{t_{1}=L}^{t} \Phi(t) \Phi^{-1}\left(t_{1}\right)\left[\left(\bigoplus_{t_{2}=L}^{t_{1}+1} \Phi\left(t_{1}\right) \Phi^{-1}\left(t_{2}\right) \hat{R}\left(t_{2}\right) \Phi\left(t_{2}\right) \Phi^{-1}\left(t_{1}\right)\right) \hat{R}\left(t_{1}\right)\right] \Phi\left(t_{1}\right) \Phi^{-1}(t) .
\end{aligned}
$$

Compare to (2.2.24), the $(\alpha, \gamma)$ term of (2.2.28) is

$$
\bigoplus_{t_{1}=l_{\alpha \gamma}}^{t} \sum_{\beta=1}^{n}\left(\left[\bigoplus_{t_{2}=l_{\alpha \beta}}^{t_{1}+1} \widehat{r}_{\alpha \beta}\left(t_{2}\right) \coprod_{t_{2}}^{t_{1}} \frac{\lambda_{\alpha}}{\lambda_{\beta}}\right] \widehat{r}_{\beta \nu}\left(t_{1}\right) \coprod_{t_{1}}^{t} \frac{\lambda_{\alpha}}{\lambda_{\gamma}}\right) .
$$




\subsection{A framework for asymptotic approximation}

The existence of the asymptotic representation (2.1.5) depends on the availability of a solution $Q(t)$ of (2.2.16) such that $Q(t) \rightarrow 0$ as $t \rightarrow \infty$. This will be provided in the following general principle. Our later tasks will be to show that (i) it may be reduced to practical criteria and (ii) it includes some known results about asymptotic summation.

THEOREM 2.3.1. If there exists constants $l_{\alpha \beta} \leq \infty$ such that for all $\alpha, \beta, \gamma, \nu$, with $\alpha \neq \beta, \beta \neq \nu$,

$$
\begin{aligned}
& \bigoplus_{t_{1}=l_{\alpha \beta}}^{t} \widehat{r}_{\alpha \beta}\left(t_{1}\right) \coprod_{t_{1}}^{t} \frac{\lambda_{\alpha}}{\lambda_{\beta}} \rightarrow 0, \\
& \bigoplus_{t_{1}=l_{\alpha \gamma}}^{t}\left|\left(\bigoplus_{t_{2}=l_{\alpha \beta}}^{t_{1}+1} \widehat{r}_{\alpha \beta}\left(t_{2}\right) \coprod_{t_{2}}^{t_{1}} \frac{\lambda_{\alpha}}{\lambda_{\beta}}\right) \widehat{r}_{\beta \nu}\left(t_{1}\right) \coprod_{t_{1}}^{t} \frac{\lambda_{\alpha}}{\lambda_{\gamma}}\right| \rightarrow 0
\end{aligned}
$$

as $t \rightarrow \infty$, then Eq. (2.1.2) has an asymptotic representation (2.1.5) where $Q(t)$ is a solution of $E q$. (2.2.16) such that $Q(t) \rightarrow 0$ as $t \rightarrow \infty$.

PRoOF. Recall that $R(t)$ is an off-diagonal matrix, so $\widehat{r}_{\alpha \alpha}=0$.

Notice that conditions (2.3.1) and (2.3.2) ensure that the elements of $\Phi(t) M_{1}(t) \Phi^{-1}(t)$ and $\Phi(t) M_{2}(t) \Phi^{-1}(t)$ of the equation (2.2.16) tends to 0 as $t \rightarrow \infty$. Consequently we multiply (2.2.16) from the left-hand side by the inverse matrix $S(t)=\left(I-\Phi(t) M_{1}(t) \Phi^{-1}(t)\right)^{-1}$ and get

$$
Q(t)=V(t)+\mathcal{L}[Q](t)
$$

where

$$
\mathcal{L}[Q](t)=-S(t) \bigoplus_{t_{1}=L}^{t} \Phi(t)\left[M_{1}\left(t_{1}+1\right) K\left(t_{1}\right) \Phi^{-1}\left(t_{1}\right) Q\left(t_{1}\right) \Phi\left(t_{1}\right)\right] \Phi^{-1}(t)
$$

and

$$
V(t)=S(t)\left[\Phi(t) M_{1}(t) \Phi^{-1}(t)-\Phi(t) M_{2}(t) \Phi^{-1}(t)\right] .
$$

Since $S(t) \rightarrow I, \Phi(t) M_{1}(t) \Phi^{-1}(t)$ and $\Phi(t) M_{2}(t) \Phi^{-1}(t) \rightarrow 0$, we have that $V(t) \rightarrow 0$ as $t \rightarrow \infty$.

we suppose that $Q(t)$ is indeed a bounded solution of (2.2.16) on some interval $[a, \infty)$. For a bounded matrix valued function $A(t)$ let $\|A(t)\|=\sum_{j k}\left|a_{j k}(t)\right|$ and let

$$
\|A\|\left\|=\sup _{t \in[a, \infty)}\right\| A(t) \| .
$$

In the equation (2.3.3) the term $q_{\gamma \nu}$ of $Q$ appear inside summation of the type (2.2.27). We bound (2.2.27) from above by

$$
\sum_{\beta=1}^{n}\left|\left(\bigoplus_{t_{1}=l_{\alpha \gamma}}^{t}\left|\left[\bigoplus_{t_{2}=l_{\alpha \beta}}^{t_{1}+1} \widehat{r}_{\alpha \beta}\left(t_{2}\right) \coprod_{t_{2}}^{t_{1}} \frac{\lambda_{\alpha}}{\lambda_{\beta}}\right] \widehat{r}_{\beta \nu}\left(t_{1}\right) \coprod_{t_{1}}^{t} \frac{\lambda_{\alpha}}{\lambda_{\gamma}}\right|\right)\right| \times|\|Q\|| \| .
$$

Denote

$$
N(t)=\max _{\alpha, \beta, \gamma, \nu}\left|\left(\bigoplus_{t_{1}=l_{\alpha \gamma}}^{t}\left|\left[\bigoplus_{t_{2}=l_{\alpha \beta}}^{t_{1}+1} \widehat{r}_{\alpha \beta}\left(t_{2}\right) \coprod_{t_{2}}^{t_{1}} \frac{\lambda_{\alpha}}{\lambda_{\beta}}\right] \widehat{r}_{\beta \nu}\left(t_{1}\right) \coprod_{t_{1}}^{t} \frac{\lambda_{\alpha}}{\lambda_{\gamma}}\right|\right)\right|,
$$


and we get from (2.3.3) that

$$
\|Q(t)\| \leq\|V(t)\|+n^{2}\|S(t)\| \cdot N(t) \cdot\|Q\| \| .
$$

Now, by (2.3.2) $N(t)$ may be made as small as we want for sufficiently large values of $t$. Take $a$ large enough so that $n^{2} \||| S|| N(t) \leq \rho<1$ on $[a, \infty)$. Then, under the present assumptions we get by $(2.3 .7)$ that

$$
\|Q\| \mid \leq \frac{\|V\| \|}{1-\rho}
$$

With these values of $a$ and $\rho$ we complete the proof of the existence of $Q(t)$ by a standard iteration. Define the sequence

$$
\begin{aligned}
& Q_{0}(t)=V(t), \\
& Q_{j}(t)=V(t)+\mathcal{L}\left[Q_{j-1}\right](t), \quad j=1,2, \ldots
\end{aligned}
$$

Then

and also

$$
\left\|Q_{j+1}(t)-Q_{j}(t)\right\|=\left\|\mathcal{L}\left[Q_{j}-Q_{j-1}\right]\right\| \leq \rho\|\| Q_{j}-Q_{j-1}\|\|
$$

$$
\left\|\left|Q_{j+1}-Q_{j}\left\|\left|\leq \rho\left\|\mid Q_{j}-Q_{j-1}\right\| \|\right.\right.\right.\right.
$$

Hence, the sequence $Q_{j}(t)$ converges uniformly on $[a, \infty)$ to a limit function $Q(t)$. It is evident that $Q(t)$ is the unique solution of the equation (2.3.3). Also, since $V(t) \rightarrow 0$ as $t \rightarrow \infty$, we get by (2.3.8) that $Q(t) \rightarrow 0$ as $t \rightarrow \infty$.

What is a reasonable choice of the limits of summation $l_{\alpha \beta}$ ? If the diagonal part $D(t)$ fits the dichotomy condition, we shall always choose the limits of summation of each term in (2.2.8), (2.2.22) and (2.2.26) so that the kernel $\coprod_{t_{1}}^{t} \frac{\lambda_{\alpha}}{\lambda_{\beta}}$ will be bounded by the corresponding dichotomy condition.

If $(\alpha, \beta)$ satisfies the dichotomy condition (1.2.11), the limit of summation of the corresponding term will be $l_{\alpha \beta}=a$. If $(\alpha, \beta)$ satisfies the dichotomy condition (1.2.12), we choose $l_{\alpha \beta}=\infty$.

The relations (2.3.1) (and (2.3.2)) demonstrate the lack of symmetry that is inherently built into Theorem 2.3.1, namely, that various perturbation terms are required to satisfy different smallness conditions. If $(\alpha, \beta)$ satisfies the dichotomy condition $(1.2 .11),((\alpha, \beta) \in$ (1.2.11), for short) then $l_{\alpha \beta}=a$ and

$$
I(t)=\bigoplus_{t_{1}=a}^{t} \widehat{r}_{\alpha \beta}\left(t_{1}\right) \coprod_{t_{1}}^{t} \frac{\lambda_{\alpha}}{\lambda_{\beta}}=\sum_{t_{1}=a}^{t-1} \widehat{r}_{\alpha \beta}\left(t_{1}\right) \prod_{t_{1}}^{t-1} \frac{\lambda_{\alpha}}{\lambda_{\beta}}
$$

has the kernel $\prod_{t_{1}}^{t-1} \frac{\lambda_{\alpha}}{\lambda_{\beta}}$ which is bounded from above by $\left|\prod_{t_{1}}^{t-1} \frac{\lambda_{\alpha}}{\lambda_{\beta}}\right| \leq K_{1}$ for $t_{1}<t$ (and tends to 0 as $t \rightarrow \infty)$. If $(\alpha, \beta)$ satisfies the dichotomy condition (1.2.12), then the summation in $(2.3 .1)$ is

$$
I(t)=\bigoplus_{t_{1}=\infty}^{t} \widehat{r}_{\alpha \beta}\left(t_{1}\right) \coprod_{t_{1}}^{t} \frac{\lambda_{\alpha}}{\lambda_{\beta}}=-\sum_{t_{1}=t+1}^{\infty} \widehat{r}_{\alpha \beta}\left(t_{1}\right) \prod_{t}^{t_{1}-1} \frac{\lambda_{\beta}}{\lambda_{\alpha}}-\widehat{r}_{\alpha \beta}(t)
$$


and for $t_{1}>t$, the kernel is $\prod_{t}^{t_{1}-1} \frac{\lambda_{\beta}}{\lambda_{\alpha}}$ which is bounded by $\left|\prod_{t}^{t_{1}-1} \frac{\lambda_{\beta}}{\lambda_{\alpha}}\right| \leq \frac{1}{K_{2}}$.

We wish to show that some known results follow, naturally, from our general work.

2.3.1. Comparison with the analog of Levinson's theorem. There is an analog in difference equations (Theorem 1.2.4) of Levinson's theorem in differential equations as presented by $[3,4]$. It claims that if the dichotomy conditions (1.2.11) and (1.2.12) hold and $\widehat{R} \in l^{1}$ then (2.1.2) has the asymptotic solution (2.1.5).

Note that in that analog of Levinson's theorem it is not assumed that $R$ is off-diagonal, as we do. However, even if the diagonal terms of $R$ are moved into $D$ and $\lambda_{i}$ is replaced by $\lambda_{i}+r_{i i}$ it makes no essential difference in the asymptotic solution (2.1.5) since $\widehat{r}_{i i}=\frac{r_{i i}}{\lambda_{i}} \in l^{1}$.

We show that this basic result can be deduced from our work. For this one must verify that (2.3.1) and (2.3.2) hold. Let us start with (2.3.1).

If $(\alpha, \beta) \in(1.2 .12)$ then $l_{\alpha \beta}=\infty$ and the summation in (2.3.1) which is also discussed in $(2.3 .10)$ can be bounded as

$$
\begin{aligned}
& \quad\left|-\sum_{t_{1}=t+1}^{\infty} \widehat{r}_{\alpha \beta}\left(t_{1}\right) \prod_{t}^{t_{1}-1} \frac{\lambda_{\beta}}{\lambda_{\alpha}}-\widehat{r}_{\alpha \beta}(t)\right| \\
& \leq \frac{1}{K_{2}}\left(\sum_{t_{1}=t+1}^{\infty}\left|\widehat{r}_{\alpha \beta}\left(t_{1}\right)\right|\right)+\left|\widehat{r}_{\alpha \beta}(t)\right| \\
& <\left(\frac{1}{K_{2}}+1\right) \sum_{t_{1}=t}^{\infty}\left|\widehat{r}_{\alpha \beta}\left(t_{1}\right)\right|
\end{aligned}
$$

since $\left|\prod_{t}^{t_{1}-1} \frac{\lambda_{\beta}}{\lambda_{\alpha}}\right| \leq \frac{1}{K_{2}}$ for $t<t_{1}$. When $\widehat{R} \in l^{1}, \sum_{t_{1}=t}^{\infty}\left|\widehat{r}_{\alpha \beta}\left(t_{1}\right)\right| \rightarrow 0$ as $t \rightarrow \infty$, which leads to

$$
-\sum_{t_{1}=t+1}^{\infty} \widehat{r}_{\alpha \beta}\left(t_{1}\right) \prod_{t}^{t_{1}-1} \frac{\lambda_{\beta}}{\lambda_{\alpha}}-\widehat{r}_{\alpha \beta}(t) \rightarrow 0 \quad \text { as } \quad t \rightarrow \infty .
$$

If $(\alpha, \beta) \in(1.2 .11)$, we formulate the calculation of (2.3.1) as a lemma for further applications.

LEMMA 2.3.2. If $(\alpha, \beta) \in(1.2 .11)$ and $\widehat{r}_{\alpha \beta}(t) \in l^{1}$ then

$$
\sum_{t_{1}=a}^{t-1}\left|\widehat{r}_{\alpha \beta}\left(t_{1}\right) \prod_{t_{1}}^{t-1} \frac{\lambda_{\alpha}}{\lambda_{\beta}}\right| \rightarrow 0 \text { as } t \rightarrow \infty \text {. }
$$

ProOF. Here $a \leq t_{1}<t<\infty$ and $\left|\prod_{t_{1}}^{t-1} \frac{\lambda_{\alpha}}{\lambda_{\beta}}\right| \leq K_{1}$. We split the summation into two parts

$$
I_{1}+I_{2}=\sum_{t_{1}=a}^{T}\left|\widehat{r}_{\alpha \beta}\left(t_{1}\right) \prod_{t_{1}}^{t-1} \frac{\lambda_{\alpha}}{\lambda_{\beta}}\right|+\sum_{t_{1}=T+1}^{t-1}\left|\widehat{r}_{\alpha \beta}\left(t_{1}\right) \prod_{t_{1}}^{t-1} \frac{\lambda_{\alpha}}{\lambda_{\beta}}\right| .
$$

For any given $\varepsilon>0$ we choose a fixed $T$ such that

$$
I_{2} \leq \sum_{t_{1}=T+1}^{t-1}\left|\widehat{r}_{\alpha \beta}\left(t_{1}\right)\right| K_{1} \leq \frac{\varepsilon}{2}
$$


for all $t \geq T+2$. With this fixed $T$

$$
I_{1}=\sum_{t_{1}=a}^{T}\left|\widehat{r}_{\alpha \beta}\left(t_{1}\right) \prod_{t_{1}}^{t-1} \frac{\lambda_{\alpha}}{\lambda_{\beta}}\right|=\left|\prod_{T+1}^{t-1} \frac{\lambda_{\alpha}}{\lambda_{\beta}}\right|\left(\sum_{t_{1}=a}^{T}\left|\widehat{r}_{\alpha \beta}\left(t_{1}\right) \prod_{t_{1}}^{T} \frac{\lambda_{\alpha}}{\lambda_{\beta}}\right|\right) .
$$

According to (1.2.11) the first factor in (2.3.15) converges to 0 as $t \rightarrow \infty$ while the second one is bounded by $K_{1} \sum_{t_{1}=a}^{\infty}\left|\widehat{r}_{\alpha \beta}\left(t_{1}\right)\right|$.

The summation in (2.3.2) may have four different forms:

$$
\begin{array}{r}
(i)-\sum_{t_{1}=t+1}^{\infty}\left|\left[-\sum_{t_{2}=t_{1}+1}^{\infty} \widehat{r}_{\alpha \beta}\left(t_{2}\right) \prod_{t_{1}}^{t_{2}-1} \frac{\lambda_{\beta}}{\lambda_{\alpha}}\right] \widehat{r}_{\beta \nu}\left(t_{1}\right) \prod_{t}^{t_{1}-1} \frac{\lambda_{\gamma}}{\lambda_{\alpha}}\right| \\
-||\left[-\sum_{t_{2}=t+1}^{\infty} \widehat{r}_{\alpha \beta}\left(t_{2}\right) \prod_{t}^{t_{2}-1} \frac{\lambda_{\beta}}{\lambda_{\alpha}}\right] \widehat{r}_{\beta \nu}(t) \mid
\end{array}
$$

if $(\alpha, \beta) \in(1.2 .12)$, and $(\alpha, \gamma) \in(1.2 .12)$

$$
\begin{array}{r}
(i i)-\sum_{t_{1}=t+1}^{\infty}\left|\left[\sum_{t_{2}=a}^{t_{1}-1} \widehat{r}_{\alpha \beta}\left(t_{2}\right) \prod_{t_{2}}^{t_{1}-1} \frac{\lambda_{\alpha}}{\lambda_{\beta}}+\widehat{r}_{\alpha \beta}\left(t_{1}\right)\right] \widehat{r}_{\beta \nu}\left(t_{1}\right) \prod_{t}^{t_{1}-1} \frac{\lambda_{\gamma}}{\lambda_{\alpha}}\right| \\
-\left|\left[\sum_{t_{2}=a}^{t-1} \widehat{r}_{\alpha \beta}\left(t_{2}\right) \prod_{t_{2}}^{t-1} \frac{\lambda_{\alpha}}{\lambda_{\beta}}+\widehat{r}_{\alpha \beta}(t)\right] \widehat{r}_{\beta \nu}(t)\right|
\end{array}
$$

if $(\alpha, \beta) \in(1.2 .11)$, and $(\alpha, \gamma) \in(1.2 .12)$

$$
\text { (iii) } \sum_{t_{1}=a}^{t-1}\left|\left[-\sum_{t_{2}=t_{1}+1}^{\infty} \widehat{r}_{\alpha \beta}\left(t_{2}\right) \prod_{t_{1}}^{t_{2}-1} \frac{\lambda_{\beta}}{\lambda_{\alpha}}\right] \widehat{r}_{\beta \nu}\left(t_{1}\right) \prod_{t_{1}}^{t-1} \frac{\lambda_{\alpha}}{\lambda_{\gamma}}\right|
$$

if $(\alpha, \beta) \in(1.2 .12)$, and $(\alpha, \gamma) \in(1.2 .11)$

$$
\text { (iv) } \sum_{t_{1}=a}^{t-1}\left|\left[\sum_{t_{2}=a}^{t_{1}-1} \widehat{r}_{\alpha \beta}\left(t_{2}\right) \prod_{t_{2}}^{t_{1}-1} \frac{\lambda_{\alpha}}{\lambda_{\beta}}+\widehat{r}_{\alpha \beta}\left(t_{1}\right)\right] \widehat{r}_{\beta \nu}\left(t_{1}\right) \prod_{t_{1}}^{t-1} \frac{\lambda_{\alpha}}{\lambda_{\gamma}}\right|
$$

if $(\alpha, \beta) \in(1.2 .11)$, and $(\alpha, \gamma) \in(1.2 .11)$.

For (2.3.16) and (2.3.17), $t<t_{1}<\infty$, so $t_{1} \rightarrow \infty$ as $t \rightarrow \infty$. Consequently the internal summations $\left(-\sum_{t_{2}=t_{1}+1}^{\infty} \widehat{r}_{\alpha \beta}\left(t_{2}\right) \prod_{t_{1}}^{t_{2}-1} \frac{\lambda_{\beta}}{\lambda_{\alpha}}\right) \rightarrow 0,\left(-\sum_{t_{2}=t+1}^{\infty} \widehat{r}_{\alpha \beta}\left(t_{2}\right) \prod_{t}^{t_{2}-1} \frac{\lambda_{\beta}}{\lambda_{\alpha}}\right) \rightarrow 0$, $\left(\sum_{t_{2}=a}^{t_{1}-1} \widehat{r}_{\alpha \beta}\left(t_{2}\right) \prod_{t_{2}}^{t_{1}-1} \frac{\lambda_{\alpha}}{\lambda_{\beta}}+\widehat{r}_{\alpha \beta}\left(t_{1}\right)\right) \rightarrow 0$, and $\left(\sum_{t_{2}=a}^{t-1} \widehat{r}_{\alpha \beta}\left(t_{2}\right) \prod_{t_{2}}^{t-1} \frac{\lambda_{\alpha}}{\lambda_{\beta}}+\widehat{r}_{\alpha \beta}(t)\right) \rightarrow 0$ as $t \rightarrow$ $\infty$ either as in (2.3.11) (for (2.3.16)) or by (2.3.13) (for (2.3.17)). Then the out summation follows easily.

For (2.3.18), $t_{2} \geq t_{1}+1$ and $(\alpha, \beta) \in(1.2 .12)$, so the internal summation is bounded by

$$
\left|-\sum_{t_{2}=t_{1}+1}^{\infty} \widehat{r}_{\alpha \beta}\left(t_{2}\right) \prod_{t_{1}}^{t_{2}-1} \frac{\lambda_{\beta}}{\lambda_{\alpha}}\right| \leq \sum_{t_{2}=t_{1}+1}^{\infty}\left|\widehat{r}_{\alpha \beta}\left(t_{2}\right)\right| \frac{1}{K_{2}} \leq \frac{1}{K_{2}} \sum_{t_{2}=a}^{\infty}\left|\widehat{r}_{\alpha \beta}\left(t_{2}\right)\right| .
$$

Since $(\alpha, \gamma) \in(1.2 .11), \sum_{t_{1}=a}^{t-1}\left|\widehat{r}_{\beta \nu}\left(t_{1}\right) \prod_{t_{1}}^{t-1} \frac{\lambda_{\alpha}}{\lambda_{\gamma}}\right| \rightarrow 0$ as in the Lemma 2.3.2. 
For (2.3.19), $t_{2} \leq t_{1}-1$ and $(\alpha, \beta) \in(1.2 .11)$, so the internal summation is bounded by

$$
\begin{aligned}
& \left|\sum_{t_{2}=a}^{t_{1}-1} \widehat{r}_{\alpha \beta}\left(t_{2}\right) \prod_{t_{2}}^{t_{1}-1} \frac{\lambda_{\alpha}}{\lambda_{\beta}}+\widehat{r}_{\alpha \beta}\left(t_{1}\right)\right| \\
\leq & \sum_{t_{2}=a}^{t_{1}-1}\left|\widehat{r}_{\alpha \beta}\left(t_{2}\right)\right| K_{1}+\left|\widehat{r}_{\alpha \beta}\left(t_{1}\right)\right| \\
\leq & \sum_{t_{2}=a}^{\infty}\left|\widehat{r}_{\alpha \beta}\left(t_{2}\right)\right|\left(1+K_{1}\right)
\end{aligned}
$$

and the double summation is completed as above.

2.3.2. A similar result of Hartman-Wintner and Behncke-Remling. In differential equations, Hartman and Wintner [24] proved that if

$$
\begin{aligned}
& \text { (a) }\left|\operatorname{Re}\left(\lambda_{\alpha}-\lambda_{\beta}\right)\right| \geq c>0, \quad \alpha \neq \beta, \\
& \text { (b) } R(t) \in L^{p}, \quad 1<p \leq 2,
\end{aligned}
$$

then the differential system $Y^{\prime}=(D(t)+R(t)) Y$ has the asymptotic solution $Y(t)=(I+$ $Q(t)) \exp \left(\int_{t_{0}}^{t} D(s) d s\right)$ with $Q(t) \rightarrow 0$ as $t \rightarrow \infty$.

Behncke and Remling [2] proved that if

$$
\begin{aligned}
& \text { (a) }\left|\operatorname{Re}\left(\lambda_{\alpha}-\lambda_{\beta}\right)\right| \geq c t^{-a_{\alpha \beta}}, \quad c>0, a_{\alpha \beta}<1, \alpha \neq \beta, \\
& \text { (b) } r_{\alpha \beta}(t) t^{b_{\alpha \beta}} \in L^{p}, \quad p>1, \\
& \text { (c) } p^{\prime} b_{\alpha \beta} \geq a_{\alpha}, \quad a_{\alpha}=\max _{\beta}\left\{a_{\alpha \beta}\right\}<1, \frac{1}{p^{\prime}}+\frac{1}{p}=1,
\end{aligned}
$$

then

$$
y_{\alpha}(t)=\left(e_{\alpha}+o(1)\right) \exp \int_{t_{0}}^{t}\left(\lambda_{\alpha}(s)+r_{\alpha \alpha}(s)+o\left(s^{-a_{\alpha}}\right)\right) d s .
$$

Since $o\left(s^{-a_{\alpha}}\right)$ is not necessarily in $L^{1}$, the approximation in (2.3.20) is not necessarily an asymptotic solution in the sense of left almost diagonal differential system. Nevertheless, we could get a similar result in difference equations with a strict estimate, without the $o\left(s^{-a_{\alpha}}\right)$ term, under a small variation of the conditions.

First of all, we introduce the expression $t^{\delta}$, with $\delta$ be any real number. It is called "falling factorial power", see [28], which is defined as

$$
t^{\underline{\delta}}=\frac{\Gamma(t+1)}{\Gamma(t-\delta+1)},
$$

where $\Gamma(t)$ is the Gamma function.

We can verify that if $\delta=1,2,3, \cdots$, then $t^{\underline{\delta}}=t(t-1)(t-2) \cdots(t-\delta+1)$. If $\delta=$ $-1,-2,-3, \cdots$, then $t^{-}=\frac{1}{(t+1)(t+2) \cdots(t-\delta)}$.

$t^{\delta}$ in the setting of difference equations is similar to $t^{\delta}$ in differential equations. There are some useful propositions such as

$$
\triangle t^{\underline{\delta}}=\delta \cdot t^{\frac{\delta-1}{}}
$$


see [28], and

$$
t^{\underline{\delta}} \sim t^{\delta},(t \rightarrow \infty)
$$

Indeed, we can use the definition of $t^{\underline{\delta}}$ and Stirling's formula, see [33], which is

$$
\Gamma(t) \sim e^{-t} t^{t}\left(\frac{2 \pi}{t}\right)^{1 / 2}
$$

to verify (2.3.23) as follows.

$$
\begin{aligned}
& \lim _{t \rightarrow \infty} \frac{t^{\underline{\delta}}}{t^{\delta}}=\lim _{t \rightarrow \infty} \frac{t^{\underline{\delta}}}{(t-\delta+1)^{\delta}}=\lim _{t \rightarrow \infty} \frac{\Gamma(t+1)}{\Gamma(t-\delta+1) \cdot(t-\delta+1)^{\delta}} \\
= & \lim _{t \rightarrow \infty} \frac{e^{-t-1}(t+1)^{t+1}\left(\frac{2 \pi}{t+1}\right)^{1 / 2}}{e^{-t+\delta-1}(t-\delta+1)^{t-\delta+1}\left(\frac{2 \pi}{t-\delta+1}\right)^{1 / 2} \cdot(t-\delta+1)^{\delta}} \\
= & \lim _{t \rightarrow \infty} e^{-\delta}\left(\frac{t+1}{t-\delta+1}\right)^{t+1}\left(\frac{t-\delta+1}{t+1}\right)^{1 / 2}=e^{-\delta} \lim _{t \rightarrow \infty}\left(1+\frac{\delta}{t-\delta+1}\right)^{t+1} \\
= & e^{-\delta} \lim _{t \rightarrow \infty}\left(1+\frac{\delta}{t-\delta+1}\right)^{t-\delta+1}\left(1+\frac{\delta}{t-\delta+1}\right)^{\delta} \\
= & e^{-\delta} \cdot e^{\delta}=1 .
\end{aligned}
$$

We can now establish our theorem as in the following.

THEOREM 2.3.3. If

$$
\begin{aligned}
& |\ln | \frac{\lambda_{\alpha}(t)}{\lambda_{\beta}(t)}|| \geq c t^{-a_{\alpha \beta}}, \quad c>0, a_{\alpha \beta}<1, \alpha \neq \beta, \\
& \hat{r}_{\alpha \beta}(t) t^{b_{\alpha \beta}} \in l^{p}, \text { and } \hat{r}_{\alpha \beta}(t) \rightarrow 0,
\end{aligned}
$$

and for $p>1$,

$$
\begin{aligned}
& p^{\prime} b_{\alpha \beta}>a_{\alpha \beta}, \quad \frac{1}{p^{\prime}}+\frac{1}{p}=1, \\
& p^{\prime} \min \left\{b_{\beta \nu}\right\}>\max \left\{a_{\alpha \gamma}\right\}
\end{aligned}
$$

or for $p=1$,

$$
b_{\alpha \beta}>0,
$$

then the difference equation (2.1.2) has an asymptotic solution (2.1.5). 
PROOF. If $\ln \left|\frac{\lambda_{\alpha}(t)}{\lambda_{\beta}(t)}\right| \geq c t \frac{-a_{\alpha \beta}}{}$, which means that $\left|\frac{\lambda_{\alpha}(t)}{\lambda_{\beta}(t)}\right| \geq e^{c t \frac{-a_{\alpha \beta}}{}}$, then $(\alpha, \beta) \in(1.2 .12)$ and we take $l_{\alpha \beta}=\infty$. When $t>0$, the summation in (2.3.1) is bounded from above by

$$
\begin{aligned}
& \left|\sum_{t_{1}=t+1}^{\infty} \widehat{r}_{\alpha \beta}\left(t_{1}\right) \prod_{t}^{t_{1}-1} \frac{\lambda_{\beta}}{\lambda_{\alpha}}\right|+\left|\widehat{r}_{\alpha \beta}(t)\right| \\
\leq & \sum_{t_{1}=t+1}^{\infty} \mid \widehat{r}_{\alpha \beta}\left(t_{1}\right) e^{-c \sum_{s=t}^{t_{1}-1} s \frac{-a_{\alpha \beta}}{}|+| \widehat{r}_{\alpha \beta}(t) \mid} \\
= & \sum_{t_{1}=t+1}^{\infty}\left|\widehat{r}_{\alpha \beta}\left(t_{1}\right) t_{1}^{b_{\alpha \beta}} t_{1}^{-b_{\alpha \beta}} e^{c\left(t^{\frac{-a_{\alpha \beta}+1}{2}-t_{1}}\right) /\left(-a_{\alpha \beta}+1\right)}\right|+\left|\widehat{r}_{\alpha \beta}(t)\right| \\
\leq & \left.\left(\sum_{t_{1}=t+1}^{\infty}\left|\widehat{r}_{\alpha \beta}\left(t_{1}\right) t_{1}^{b_{\alpha \beta}}\right|^{p}\right)^{1 / p}\left(\sum_{t_{1}=t+1}^{\infty} t_{1}^{-p^{\prime} b_{\alpha \beta}} e^{p^{\prime} c\left(t^{\frac{-a_{\alpha \beta}+1}{2}-t_{1}}\right) /\left(-a_{\alpha \beta}+1\right.}\right)\right)^{1 / p^{\prime}}+\left|\widehat{r}_{\alpha \beta}(t)\right| \\
(2.3 .30) & =A_{1} A_{2}+\left|\widehat{r}_{\alpha \beta}(t)\right|,
\end{aligned}
$$

with

$$
\begin{aligned}
& A_{1}=\left(\sum_{t_{1}=t+1}^{\infty}\left|\widehat{r}_{\alpha \beta}\left(t_{1}\right) t_{1}^{b_{\alpha \beta}}\right|^{p}\right)^{1 / p} \\
& A_{2}=\left(\sum_{t_{1}=t+1}^{\infty} t_{1}^{-p^{\prime} b_{\alpha \beta}} e^{p^{\prime} c\left(t^{-a_{\alpha \beta}+1}-t_{1} \frac{-a_{\alpha \beta}+1}{1}\right) /\left(-a_{\alpha \beta}+1\right)}\right)^{1 / p^{\prime}} .
\end{aligned}
$$

$A_{1} \rightarrow 0$ because of the first part of (2.3.26). For $A_{2}$, we need the asymptotic approximation

$$
\int_{t}^{\infty} s^{u} e^{\left(t^{v+1}-s^{v+1}\right) /(v+1)} d s \sim t^{u-v} \quad \text { as } t \rightarrow \infty
$$

which is equivalent to

$$
\lim _{t \rightarrow \infty} \frac{\int_{t}^{\infty} s^{u} e^{-s^{v+1} /(v+1)} d s}{t^{u-v} e^{-t^{v+1} /(v+1)}}=1
$$

an easy result by using L'Hopital's rule. Observe that

$$
\begin{aligned}
\left(t^{u} e^{-t^{v+1} /(v+1)}\right)^{\prime} & =u t^{u-1} e^{-t^{v+1} /(v+1)}-t^{u+v} e^{-t^{v+1} /(v+1)} \\
& =\left(u-t^{v+1}\right) t^{u-1} e^{-t^{v+1} /(v+1)} \\
& <0
\end{aligned}
$$

when $t>\max \left\{0, u^{1 /(v+1)}\right\}$ and $v>-1$. Therefore, when $t \rightarrow \infty$,

$$
\int_{t+1}^{\infty} s^{u} e^{-s^{v+1} /(v+1)} d s \leq \sum_{s=t+1}^{\infty} s^{u} e^{-s^{v+1} /(v+1)} \leq \int_{t}^{\infty} s^{u} e^{-s^{v+1} /(v+1)} d s
$$

which leads to

$$
\int_{t+1}^{\infty} s^{u} e^{\left(t^{v+1}-s^{v+1}\right) /(v+1)} d s<\sum_{s=t+1}^{\infty} s^{u} e^{\left(t^{v+1}-s^{v+1}\right) /(v+1)}<\int_{t}^{\infty} s^{u} e^{\left(t^{v+1}-s^{v+1}\right) /(v+1)} d s .
$$


According to (2.3.31), (2.3.32) and (2.3.27) with $u=-p^{\prime} b_{\alpha \beta}, v=-a_{\alpha \beta}(v>-1$ because of (2.3.25)), we get that $A_{2} \rightarrow 0$ as $t \rightarrow \infty$.

Thus, together with the second part of condition (2.3.26), (2.3.30) goes to 0 as $t \rightarrow \infty$.

If $\ln \left|\frac{\lambda_{\alpha}(t)}{\lambda_{\beta}(t)}\right| \leq-c t \frac{-a_{\alpha \beta}}{}$, which means that $\left|\frac{\lambda_{\alpha}(t)}{\lambda_{\beta}(t)}\right| \leq e^{-c t \frac{-a_{\alpha \beta}}{}}$, then $(\alpha, \beta) \in(1.2 .11)$ and we take $l_{\alpha \beta}=a$. Then the summation in (2.3.1) is bounded from above by

$$
\begin{aligned}
& \sum_{t_{1}=a}^{t-1}\left|\widehat{r}_{\alpha \beta}\left(t_{1}\right) \prod_{t_{1}}^{t-1} \frac{\lambda_{\alpha}}{\lambda_{\beta}}\right| \\
& \leq \sum_{t_{1}=a}^{t-1}\left|\widehat{r}_{\alpha \beta}\left(t_{1}\right)\right| e^{-c \sum_{s=t_{1}}^{t-1} s \underline{-a_{\alpha \beta}}} \\
& \left.\leq\left[\sum_{t_{1}=a}^{t-1}\left(\left|\widehat{r}_{\alpha \beta}\left(t_{1}\right)\right| t_{1}^{b_{\alpha \beta}}\right)^{p}\right]^{1 / p}\left[\sum_{t_{1}=a}^{t-1} t_{1}^{-p^{\prime} b_{\alpha \beta}} e^{-p^{\prime} c\left(t^{-a_{\alpha \beta}+1}-t_{1}^{-a_{\alpha \beta}+1}\right.}\right) /\left(-a_{\alpha \beta}+1\right)\right]^{1 / p^{\prime}} \\
& =B_{1} B_{2} \text {, }
\end{aligned}
$$

when $a>0$, with

$$
\begin{aligned}
& B_{1}=\left[\sum_{t_{1}=a}^{t-1}\left(\left|\widehat{r}_{\alpha \beta}\left(t_{1}\right)\right| t_{1}^{b_{\alpha \beta}}\right)^{p}\right]^{1 / p}, \\
& B_{2}=\left[\sum_{t_{1}=a}^{t-1} t_{1}^{-p^{\prime} b_{\alpha \beta}} e^{-p^{\prime} c\left(t \frac{-a_{\alpha \beta}+1}{-t_{1}}\right) /\left(-a_{\alpha \beta}+1\right)}\right]^{1 / p^{\prime}} .
\end{aligned}
$$

Now $B_{1}$ is bounded because of the first part of (2.3.26) and $B_{2} \rightarrow 0$ due to the estimate

$$
\int_{a}^{t} s^{u} e^{-\left(t^{v+1}-s^{v+1}\right) /(v+1)} d s \sim t^{u-v} \quad \text { as } t \rightarrow \infty
$$

and

$$
\int_{a}^{t-1} s^{u} e^{-\left(t^{v+1}-s^{v+1}\right) /(v+1)} d s \leq \sum_{s=a}^{t-1} s^{u} e^{-\left(t^{v+1}-s^{v+1}\right) /(v+1)} \leq \int_{a}^{t} s^{u} e^{-\left(t^{v+1}-s^{v+1}\right) /(v+1)} d s,
$$

which is similarly verified as in (2.3.32).

For $p=1,(2.3 .30)$ is replaced by

$$
\begin{aligned}
& \left|\sum_{t_{1}=t+1}^{\infty} \widehat{r}_{\alpha \beta}\left(t_{1}\right) \prod_{t}^{t_{1}-1} \frac{\lambda_{\beta}}{\lambda_{\alpha}}\right|+\left|\widehat{r}_{\alpha \beta}(t)\right| \\
\leq & \sum_{t_{1}=t+1}^{\infty}\left|\widehat{r}_{\alpha \beta}\left(t_{1}\right) t_{1}^{b_{\alpha \beta}}\right| \max _{t_{1} \geq t+1}\left\{t_{1}^{-b_{\alpha \beta}} e^{c\left(t^{-a_{\alpha \beta}+1}-t_{1}^{-a_{\alpha \beta}+1}\right) /\left(-a_{\alpha \beta}+1\right)}\right\}+\left|\widehat{r}_{\alpha \beta}(t)\right| \\
(2.3 .36)= & \left(\sum_{t_{1}=t+1}^{\infty}\left|\widehat{r}_{\alpha \beta}\left(t_{1}\right) t_{1}^{b_{\alpha \beta}}\right|\right)(t+1)^{-b_{\alpha \beta}} e^{c\left(t^{-a_{\alpha \beta}+1}-(t+1) \frac{-a_{\alpha \beta}+1}{2}\right) /\left(-a_{\alpha \beta}+1\right)}+\left|\widehat{r}_{\alpha \beta}(t)\right|,
\end{aligned}
$$

because $-a_{\alpha \beta}+1>0$. Hence, when $b_{\alpha \beta}>0$, (2.3.36) approaches to 0 .

The replacement of (2.3.33) also approaches 0 that could be showed similarly in this case. 
The summation (2.3.2) may again have the four forms (2.3.16) - (2.3.19) and it is treated as in our proof of comparison of the analog of Levinson's theorem. First we show that the internal summations either tend to 0 or are, at least, bounded. At the second step we see that the double summation tend to 0 if $p^{\prime} b_{\beta \nu}>a_{\alpha \gamma}$, i.e., if

$$
p^{\prime} \min \left\{b_{\beta \nu}\right\}>\max \left\{a_{\alpha \gamma}\right\},
$$

or $b_{\beta \nu}>0$ in the case $p=1$.

The following corollary is easy to be formalized whose proof is now superfluous.

COROllary 2.3.4. If

$$
\begin{aligned}
& \text { (a) }|\ln | \frac{\lambda_{\alpha}(t)}{\lambda_{\beta}(t)}|| \geq c t^{-a}, \quad c>0, a<1, \alpha \neq \beta, \\
& \text { (b) } \hat{r}_{\alpha \beta}(t) t^{b} \in l^{p}, \quad p>1, \text { and } \hat{r}_{\alpha \beta}(t) \rightarrow 0, \\
& \text { (c) } p^{\prime} b>a, \frac{1}{p^{\prime}}+\frac{1}{p}=1
\end{aligned}
$$

or if

$$
\begin{aligned}
& \text { (a) }|\ln | \frac{\lambda_{\alpha}(t)}{\lambda_{\beta}(t)}|| \geq c t^{-a}, c>0, a<1, \alpha \neq \beta, \\
& \text { (b) } \hat{r}_{\alpha \beta}(t) t^{b} \in l^{1}, b>0,
\end{aligned}
$$

then the difference equation (2.1.2) has an asymptotic solution (2.1.5).

\subsection{An explicit criteria}

Theorem 2.3.1 formulates a general principle for asymptotic summation. In this section we obtain an explicit, simple criteria which will enable us to verify that the assumptions (2.3.1) and (2.3.2) really hold. This is done through systematic summation by parts.

THEOREM 2.4.1. The conditions of Theorem 3.1 hold under the following assumptions: for a difference system (2.1.2) that satisfies the dichotomy condition and for every $\alpha \neq \beta, \nu$

$$
\begin{aligned}
& \frac{\widehat{r}_{\alpha \beta}(t)}{\frac{\lambda_{\beta}}{\lambda_{\alpha}}(t)-1}=\frac{r_{\alpha \beta}(t)}{\lambda_{\beta}(t)-\lambda_{\alpha}(t)} \rightarrow 0, \text { and } \widehat{r}_{\alpha \beta}(t) \rightarrow 0 \quad \text { as } t \rightarrow \infty, \\
& \triangle\left(\frac{\widehat{r}_{\alpha \beta}(t)}{\frac{\lambda_{\beta}}{\lambda_{\alpha}}(t)-1}\right) \in l^{1}, \\
& \left(\sum_{t_{2}=t-1}^{\infty}\left|\triangle_{t_{2}} \frac{\widehat{r}_{\alpha \beta}\left(t_{2}\right)}{\frac{\lambda_{\beta}}{\lambda_{\alpha}}\left(t_{2}\right)-1}\right|\right)\left|\widehat{r}_{\beta \nu}(t)\right| \in l^{1},
\end{aligned}
$$

and for $(\alpha, \beta)$ satisfies $(1.2 .11)$, also

$$
\begin{aligned}
& \widehat{r}_{\beta \nu}(t) \prod_{a}^{t-1} \frac{\lambda_{\alpha}}{\lambda_{\beta}} \in l^{1}, \quad \widehat{r}_{\alpha \beta}(t-1) \widehat{r}_{\beta \nu}(t) \in l^{1}, \text { and } \quad \widehat{r}_{\alpha \beta}(t) \widehat{r}_{\beta \nu}(t) \in l^{1}, \\
& \widehat{r}_{\beta \nu}(t) \sum_{t_{2}=a}^{t-2}\left[\left(\triangle_{t_{2}} \frac{\widehat{r}_{\alpha \beta}\left(t_{2}\right)}{\frac{\lambda_{\beta}}{\lambda_{\alpha}}\left(t_{2}\right)-1}\right) \prod_{t_{2}+1}^{t-1} \frac{\lambda_{\alpha}}{\lambda_{\beta}}\right] \in l^{1} .
\end{aligned}
$$


Proof. We begin to show that (2.4.1) and (2.4.2) imply (2.3.1).

If $(\alpha, \beta) \in(1.2 .11)$ then $l_{\alpha \beta}=a$, the summation in (2.3.1) is

$$
\sum_{t_{1}=a}^{t-1} \widehat{r}_{\alpha \beta}\left(t_{1}\right) \prod_{t_{1}}^{t-1} \frac{\lambda_{\alpha}}{\lambda_{\beta}}=\sum_{t_{1}=a}^{t-2} \widehat{r}_{\alpha \beta}\left(t_{1}\right) \prod_{t_{1}}^{t-1} \frac{\lambda_{\alpha}}{\lambda_{\beta}}+\widehat{r}_{\alpha \beta}(t-1) \frac{\lambda_{\alpha}}{\lambda_{\beta}}(t-1)
$$

as showed in (2.3.9).

When $t_{1}<t-1$,

$$
\triangle_{t_{1}} \prod_{t_{1}}^{t-1} \frac{\lambda_{\alpha}}{\lambda_{\beta}}=\prod_{t_{1}+1}^{t-1} \frac{\lambda_{\alpha}}{\lambda_{\beta}}-\prod_{t_{1}}^{t-1} \frac{\lambda_{\alpha}}{\lambda_{\beta}}=\left(\frac{\lambda_{\beta}}{\lambda_{\alpha}}\left(t_{1}\right)-1\right) \prod_{t_{1}}^{t-1} \frac{\lambda_{\alpha}}{\lambda_{\beta}} .
$$

Therefore, using summation by parts, (2.4.6) becomes

$$
\begin{aligned}
& \sum_{t_{1}=a}^{t-2} \frac{\widehat{r}_{\alpha \beta}\left(t_{1}\right)}{\frac{\lambda_{\beta}}{\lambda_{\alpha}}\left(t_{1}\right)-1} \triangle_{t_{1}} \prod_{t_{1}}^{t-1} \frac{\lambda_{\alpha}}{\lambda_{\beta}}+\widehat{r}_{\alpha \beta}(t-1) \frac{\lambda_{\alpha}}{\lambda_{\beta}}(t-1) \\
= & {\left[\frac{\widehat{r}_{\alpha \beta}\left(t_{1}\right)}{\frac{\lambda_{\beta}}{\lambda_{\alpha}}\left(t_{1}\right)-1} \prod_{t_{1}}^{t-1} \frac{\lambda_{\alpha}}{\lambda_{\beta}}\right]_{a}^{t-1}-\sum_{t_{1}=a}^{t-2}\left(\triangle_{t_{1}} \frac{\widehat{r}_{\alpha \beta}\left(t_{1}\right)}{\frac{\lambda_{\beta}}{\lambda_{\alpha}}\left(t_{1}\right)-1}\right) \prod_{t_{1}+1}^{t-1} \frac{\lambda_{\alpha}}{\lambda_{\beta}}+\widehat{r}_{\alpha \beta}(t-1) \frac{\lambda_{\alpha}}{\lambda_{\beta}}(t-1) } \\
= & \frac{\widehat{r}_{\alpha \beta}(t-1)}{\frac{\lambda_{\beta}}{\lambda_{\alpha}}(t-1)-1} \cdot \frac{\lambda_{\alpha}}{\lambda_{\beta}}(t-1)-\frac{\widehat{r}_{\alpha \beta}(a)}{\frac{\lambda_{\beta}}{\lambda_{\alpha}}(a)-1} \prod_{a}^{t-1} \frac{\lambda_{\alpha}}{\lambda_{\beta}} \\
& \quad-\sum_{t_{1}=a}^{t-2}\left(\triangle_{t_{1}} \frac{\widehat{r}_{\alpha \beta}\left(t_{1}\right)}{\frac{\lambda_{\beta}}{\lambda_{\alpha}}\left(t_{1}\right)-1}\right) \prod_{t_{1}+1}^{t-1} \frac{\lambda_{\alpha}}{\lambda_{\beta}}+\widehat{r}_{\alpha \beta}(t-1) \frac{\lambda_{\alpha}}{\lambda_{\beta}}(t-1) .
\end{aligned}
$$

The first term of (2.4.8) goes to 0 by the first part of (2.4.1), the second by (1.2.11), the third by (2.4.2) and Lemma 2.3.2, and the forth by the second part of (2.4.1).

If $(\alpha, \beta) \in(1.2 .12)$, then $l_{\alpha \beta}=\infty$, the summation in $(2.3 .1)$ is

$$
-\sum_{t_{1}=t+1}^{\infty} \widehat{r}_{\alpha \beta}\left(t_{1}\right) \prod_{t}^{t_{1}-1} \frac{\lambda_{\beta}}{\lambda_{\alpha}}-\widehat{r}_{\alpha \beta}(t)
$$

as showed in (2.3.10). Since

$$
\triangle_{t_{1}} \prod_{t}^{t_{1}-1} \frac{\lambda_{\beta}}{\lambda_{\alpha}}=\left(\frac{\lambda_{\beta}}{\lambda_{\alpha}}\left(t_{1}\right)-1\right) \prod_{t}^{t_{1}-1} \frac{\lambda_{\beta}}{\lambda_{\alpha}}
$$


using summation by parts, (2.4.9) turns to

$$
\begin{aligned}
& -\sum_{t_{1}=t+1}^{\infty} \frac{\widehat{r}_{\alpha \beta}\left(t_{1}\right)}{\frac{\lambda_{\beta}}{\lambda_{\alpha}}\left(t_{1}\right)-1}\left(\triangle_{t_{1}} \prod_{t}^{t_{1}-1} \frac{\lambda_{\beta}}{\lambda_{\alpha}}\right)-\widehat{r}_{\alpha \beta}(t) \\
= & -\left[\frac{\widehat{r}_{\alpha \beta}\left(t_{1}\right)}{\frac{\lambda_{\beta}}{\lambda_{\alpha}}\left(t_{1}\right)-1} \prod_{t}^{t_{1}-1} \frac{\lambda_{\beta}}{\lambda_{\alpha}}\right]_{t+1}^{\infty}+\sum_{t_{1}=t+1}^{\infty} \triangle_{t_{1}} \frac{\widehat{r}_{\alpha \beta}\left(t_{1}\right)}{\frac{\lambda_{\beta}}{\lambda_{\alpha}}\left(t_{1}\right)-1} \prod_{t}^{t_{1}} \frac{\lambda_{\beta}}{\lambda_{\alpha}}-\widehat{r}_{\alpha \beta}(t) \\
= & -\frac{\widehat{r}_{\alpha \beta}(\infty)}{\frac{\lambda_{\beta}}{\lambda_{\alpha}}(\infty)-1} \prod_{t}^{\infty} \frac{\lambda_{\beta}}{\lambda_{\alpha}}+\frac{\widehat{r}_{\alpha \beta}(t+1)}{\frac{\lambda_{\beta}}{\lambda_{\alpha}}(t+1)-1} \cdot \frac{\lambda_{\beta}}{\lambda_{\alpha}}(t) \\
& +\sum_{t_{1}=t+1}^{\infty} \triangle_{t_{1}} \frac{\widehat{r}_{\alpha \beta}\left(t_{1}\right)}{\frac{\lambda_{\beta}}{\lambda_{\alpha}}\left(t_{1}\right)-1} \prod_{t}^{t_{1}} \frac{\lambda_{\beta}}{\lambda_{\alpha}}-\widehat{r}_{\alpha \beta}(t) .
\end{aligned}
$$

As $\left|\prod_{t}^{\infty} \frac{\lambda_{\beta}}{\lambda_{\alpha}}\right|$ is bounded by $\frac{1}{K_{2}}$, the first term is 0 by the first part of (2.4.1), the second term tends to 0 also by the first part of (2.4.1), the third one tents to 0 by (2.4.2) and the last term by the second part of (2.4.1).

Next we show that (2.3.2) is implied by (2.4.3)-(2.4.5). The discussion of (2.3.2) is divided again into four cases as in (2.3.16)-(2.3.19).

Case I. $(\alpha, \beta) \in(1.2 .12)$, and $(\alpha, \gamma) \in(1.2 .12)$. In this case, the summation in (2.3.2) is

$$
\begin{aligned}
& S_{1}(t) \\
& =-\sum_{t_{1}=t+1}^{\infty}\left|\left[-\sum_{t_{2}=t_{1}+1}^{\infty} \widehat{r}_{\alpha \beta}\left(t_{2}\right) \prod_{t_{1}}^{t_{2}-1} \frac{\lambda_{\beta}}{\lambda_{\alpha}}\right] \widehat{r}_{\beta \nu}\left(t_{1}\right) \prod_{t}^{t_{1}-1} \frac{\lambda_{\gamma}}{\lambda_{\alpha}}\right|-\left|\left\lceil-\sum_{t_{2}=t+1}^{\infty} \widehat{r}_{\alpha \beta}\left(t_{2}\right) \prod_{t}^{t_{2}-1} \frac{\lambda_{\beta}}{\lambda_{\alpha}}\right] \widehat{r}_{\beta \nu}(t)\right| \\
& =-\sum_{t_{1}=t+1}^{\infty}\left|\left[\frac{\widehat{r}_{\alpha \beta}\left(t_{1}+1\right)}{\frac{\lambda_{\beta}}{\lambda_{\alpha}}\left(t_{1}+1\right)-1} \cdot \frac{\lambda_{\beta}}{\lambda_{\alpha}}\left(t_{1}\right)+\sum_{t_{2}=t_{1}+1}^{\infty}\left(\triangle_{t_{2}} \frac{\widehat{r}_{\alpha \beta}\left(t_{2}\right)}{\frac{\lambda_{\beta}}{\lambda_{\alpha}}\left(t_{2}\right)-1}\right) \prod_{t_{1}}^{t_{2}} \frac{\lambda_{\beta}}{\lambda_{\alpha}}\right] \cdot \widehat{r}_{\beta \nu}\left(t_{1}\right) \prod_{t}^{t_{1}-1} \frac{\lambda_{\gamma}}{\lambda_{\alpha}}\right| \\
& -\left|\left[-\frac{\widehat{r}_{\alpha \beta}(t+1)}{\frac{\lambda_{\beta}}{\lambda_{\alpha}}(t+1)-1} \cdot \frac{\lambda_{\beta}}{\lambda_{\alpha}}(t)+\sum_{t_{2}=t+1}^{\infty}\left(\triangle_{t_{2}} \frac{\widehat{r}_{\alpha \beta}\left(t_{2}\right)}{\frac{\lambda_{\beta}}{\lambda_{\alpha}}\left(t_{2}\right)-1}\right) \prod_{t}^{t_{2}} \frac{\lambda_{\beta}}{\lambda_{\alpha}}\right] \widehat{r}_{\beta \nu}(t)\right| .
\end{aligned}
$$

Here, $\left|\frac{\lambda_{\beta}}{\lambda_{\alpha}}\left(t_{1}\right)\right|,\left|\prod_{t_{1}}^{t_{2}} \frac{\lambda_{\beta}}{\lambda_{\alpha}}\right|,\left|\frac{\lambda_{\beta}}{\lambda_{\alpha}}(t)\right|,\left|\prod_{t}^{t_{2}} \frac{\lambda_{\beta}}{\lambda_{\alpha}}\right|$ and $\left|\prod_{t}^{t_{1}-1} \frac{\lambda_{\gamma}}{\lambda_{\alpha}}\right|$ are all bounded by $\frac{1}{K_{2}}$.

Thanks to (2.4.1),

$$
\left|\frac{\widehat{r}_{\alpha \beta}\left(t_{1}+1\right)}{\frac{\lambda_{\beta}}{\lambda_{\alpha}}\left(t_{1}+1\right)-1}\right|=\left|\sum_{t_{2}=t_{1}+1}^{\infty} \triangle_{t_{2}} \frac{\widehat{r}_{\alpha \beta}\left(t_{2}\right)}{\frac{\lambda_{\beta}}{\lambda_{\alpha}}\left(t_{2}\right)-1}\right| \leq \sum_{t_{2}=t_{1}+1}^{\infty}\left|\triangle_{t_{2}} \frac{\widehat{r}_{\alpha \beta}\left(t_{2}\right)}{\frac{\lambda_{\beta}}{\lambda_{\alpha}}\left(t_{2}\right)-1}\right| .
$$

Therefore, $\left|S_{1}(t)\right|$ in $(2.4 .12)$ can be bounded by

$$
\begin{aligned}
& \sum_{t_{1}=t+1}^{\infty}\left[\sum_{t_{2}=t_{1}+1}^{\infty}\left|\triangle_{t_{2}} \frac{\widehat{r}_{\alpha \beta}\left(t_{2}\right)}{\frac{\lambda_{\beta}}{\lambda_{\alpha}}\left(t_{2}\right)-1}\right| \cdot \frac{1}{K_{2}}+\sum_{t_{2}=t_{1}+1}^{\infty}\left|\triangle_{t_{2}} \frac{\widehat{r}_{\alpha \beta}\left(t_{2}\right)}{\frac{\lambda_{\beta}}{\lambda_{\alpha}}\left(t_{2}\right)-1}\right| \cdot \frac{1}{K_{2}}\right]\left|\widehat{r}_{\beta \nu}\left(t_{1}\right)\right| \frac{1}{K_{2}} \\
& +\left[\sum_{t_{2}=t+1}^{\infty}\left|\triangle_{t_{2}} \frac{\widehat{r}_{\alpha \beta}\left(t_{2}\right)}{\frac{\lambda_{\beta}}{\lambda_{\alpha}}\left(t_{2}\right)-1}\right| \cdot \frac{1}{K_{2}}+\sum_{t_{2}=t+1}^{\infty}\left|\triangle_{t_{2}} \frac{\widehat{r}_{\alpha \beta}\left(t_{2}\right)}{\frac{\lambda_{\beta}}{\lambda_{\alpha}}\left(t_{2}\right)-1}\right| \cdot \frac{1}{K_{2}}\right]\left|\widehat{r}_{\beta \nu}(t)\right| \\
\leq & M_{1} \sum_{t_{1}=t}^{\infty}\left[\frac{2}{K_{2}} \sum_{t_{2}=t_{1}+1}^{\infty}\left|\triangle_{t_{2}} \frac{\widehat{r}_{\alpha \beta}\left(t_{2}\right)}{\frac{\lambda_{\beta}}{\lambda_{\alpha}}\left(t_{2}\right)-1}\right|\right]\left|\widehat{r}_{\beta \nu}\left(t_{1}\right)\right|,
\end{aligned}
$$


where $M_{1}=\max \left\{1, \frac{1}{K_{2}}\right\}$. Assumption (2.4.3) guarantees that $S_{1}(t)$ tends to 0 since

$$
\sum_{t_{2}=t_{1}+1}^{\infty}\left|\triangle_{t_{2}} \frac{\widehat{r}_{\alpha \beta}\left(t_{2}\right)}{\frac{\lambda_{\beta}}{\lambda_{\alpha}}\left(t_{2}\right)-1}\right|<\sum_{t_{2}=t_{1}-1}^{\infty}\left|\triangle_{t_{2}} \frac{\widehat{r}_{\alpha \beta}\left(t_{2}\right)}{\frac{\lambda_{\beta}}{\lambda_{\alpha}}\left(t_{2}\right)-1}\right| .
$$

Case II. $(\alpha, \beta) \in(1.2 .12)$, and $(\alpha, \gamma) \in(1.2 .11)$. In this case, (2.3.2) is

$$
\sum_{t_{1}=a}^{t-1}\left|\left[-\sum_{t_{2}=t_{1}+1}^{\infty} \widehat{r}_{\alpha \beta}\left(t_{2}\right) \prod_{t_{1}}^{t_{2}-1} \frac{\lambda_{\beta}}{\lambda_{\alpha}}\right] \widehat{r}_{\beta \nu}\left(t_{1}\right) \prod_{t_{1}}^{t-1} \frac{\lambda_{\alpha}}{\lambda_{\gamma}}\right| .
$$

Since $(\alpha, \gamma) \in(1.2 .11)$, it is sufficient according to Lemma 2.3.2 to require that the summation (considered as a function of $t_{1}$ ) satisfies

$$
\left[-\sum_{t_{2}=t_{1}+1}^{\infty} \widehat{r}_{\alpha \beta}\left(t_{2}\right) \prod_{t_{1}}^{t_{2}-1} \frac{\lambda_{\beta}}{\lambda_{\alpha}}\right] \widehat{r}_{\beta \nu}\left(t_{1}\right) \in l^{1}
$$

Similar to Case I, using summation by parts (2.4.14) can be bounded by

$$
\begin{aligned}
& \left|\left[\frac{\widehat{r}_{\alpha \beta}\left(t_{1}+1\right)}{\frac{\lambda_{\beta}}{\lambda_{\alpha}}\left(t_{1}+1\right)-1} \cdot \frac{\lambda_{\beta}}{\lambda_{\alpha}}\left(t_{1}\right)+\sum_{t_{2}=t_{1}+1}^{\infty}\left(\triangle_{t_{2}} \frac{\widehat{r}_{\alpha \beta}\left(t_{2}\right)}{\frac{\lambda_{\beta}}{\lambda_{\alpha}}\left(t_{2}\right)-1}\right) \prod_{t_{1}}^{t_{2}} \frac{\lambda_{\beta}}{\lambda_{\alpha}}\right] \widehat{r}_{\beta \nu}\left(t_{1}\right)\right| \\
\leq & {\left[\left(\sum_{t_{2}=t_{1}+1}^{\infty}\left|\triangle_{t_{2}} \frac{\widehat{r}_{\alpha \beta}\left(t_{2}\right)}{\frac{\lambda_{\beta}}{\lambda_{\alpha}}\left(t_{2}\right)-1}\right|\right)\left|\frac{\lambda_{\beta}}{\lambda_{\alpha}}\left(t_{1}\right)\right|+\sum_{t_{2}=t_{1}+1}^{\infty}\left|\triangle_{t_{2}} \frac{\widehat{r}_{\alpha \beta}\left(t_{2}\right)}{\frac{\lambda_{\beta}}{\lambda_{\alpha}}\left(t_{2}\right)-1}\right| \frac{1}{K_{2}}\right]\left|\widehat{r}_{\beta \nu}\left(t_{1}\right)\right|, }
\end{aligned}
$$

which is belong to $l^{1}$ by the assumption (2.4.3). Here we also use (2.4.13) in our estimation.

Case III. $(\alpha, \beta) \in(1.2 .11)$, and $(\alpha, \gamma) \in(1.2 .12)$. In this case (2.3.2) is

$$
\begin{aligned}
-\sum_{t_{1}=t+1}^{\infty} \mid & \left|\left[\sum_{t_{2}=a}^{t_{1}-1} \widehat{r}_{\alpha \beta}\left(t_{2}\right) \prod_{t_{2}}^{t_{1}-1} \frac{\lambda_{\alpha}}{\lambda_{\beta}}+\widehat{r}_{\alpha \beta}\left(t_{1}\right)\right] \widehat{r}_{\beta \nu}\left(t_{1}\right) \prod_{t}^{t_{1}-1} \frac{\lambda_{\gamma}}{\lambda_{\alpha}}\right| \\
- & \left|\left[\sum_{t_{2}=a}^{t-1} \widehat{r}_{\alpha \beta}\left(t_{2}\right) \prod_{t_{2}}^{t-1} \frac{\lambda_{\alpha}}{\lambda_{\beta}}+\widehat{r}_{\alpha \beta}(t)\right] \widehat{r}_{\beta \nu}(t)\right| .
\end{aligned}
$$


Similar to the summation by parts to (2.4.6), it is bounded by

$$
\begin{aligned}
\sum_{t_{1}=t+1}^{\infty} \mid\left[\frac{\widehat{r}_{\alpha \beta}\left(t_{1}-1\right)}{\frac{\lambda_{\beta}}{\lambda_{\alpha}}\left(t_{1}-1\right)-1} \cdot \frac{\lambda_{\alpha}}{\lambda_{\beta}}\left(t_{1}-1\right)-\frac{\widehat{r}_{\alpha \beta}(a)}{\frac{\lambda_{\beta}}{\lambda_{\alpha}}(a)-1} \cdot \prod_{a}^{t_{1}-1} \frac{\lambda_{\alpha}}{\lambda_{\beta}}\right. \\
\left.-\sum_{t_{2}=a}^{t_{1}-2}\left(\triangle_{t_{2}} \frac{\widehat{r}_{\alpha \beta}\left(t_{2}\right)}{\frac{\lambda_{\beta}}{\lambda_{\alpha}}\left(t_{2}\right)-1}\right) \prod_{t_{2}+1}^{t_{1}-1} \frac{\lambda_{\alpha}}{\lambda_{\beta}}+\widehat{r}_{\alpha \beta}\left(t_{1}-1\right) \frac{\lambda_{\alpha}}{\lambda_{\beta}}\left(t_{1}-1\right)+\widehat{r}_{\alpha \beta}\left(t_{1}\right)\right] \cdot \widehat{r}_{\beta \nu}\left(t_{1}\right) \prod_{t}^{t_{1}-1} \frac{\lambda_{\gamma}}{\lambda_{\alpha}} \mid \\
+\mid\left[\frac{\widehat{r}_{\alpha \beta}(t-1)}{\frac{\lambda_{\beta}}{\lambda_{\alpha}}(t-1)-1} \cdot \frac{\lambda_{\alpha}}{\lambda_{\beta}}(t-1)-\frac{\widehat{r}_{\alpha \beta}(a)}{\frac{\lambda_{\beta}}{\lambda_{\alpha}}(a)-1} \cdot \prod_{a}^{t-1} \frac{\lambda_{\alpha}}{\lambda_{\beta}}-\sum_{t_{2}=a}^{t-2}\left(\triangle_{t_{2}} \frac{\widehat{r}_{\alpha \beta}\left(t_{2}\right)}{\frac{\lambda_{\beta}}{\lambda_{\alpha}}\left(t_{2}\right)-1}\right) \prod_{t_{2}+1}^{t-1} \frac{\lambda_{\alpha}}{\lambda_{\beta}}\right. \\
\left.+\quad \widehat{r}_{\alpha \beta}(t-1) \frac{\lambda_{\alpha}}{\lambda_{\beta}}(t-1)+\widehat{r}_{\alpha \beta}\left(t_{1}\right)\right] \widehat{r}_{\beta \nu}(t) \mid \\
\leq M_{1} \sum_{t_{1}=t}^{\infty} \mid\left[\frac{\widehat{r}_{\alpha \beta}\left(t_{1}-1\right)}{\frac{\lambda_{\beta}}{\lambda_{\alpha}}\left(t_{1}-1\right)-1} \cdot \frac{\lambda_{\alpha}}{\lambda_{\beta}}\left(t_{1}-1\right)-\frac{\widehat{r}_{\alpha \beta}(a)}{\frac{\lambda_{\beta}}{\lambda_{\alpha}}(a)-1} \cdot \prod_{a}^{t_{1}-1} \frac{\lambda_{\alpha}}{\lambda_{\beta}}\right. \\
\left.\quad-\sum_{t_{2}=a}^{t_{1}-2}\left(\triangle_{t_{2}} \frac{\widehat{r}_{\alpha \beta}\left(t_{2}\right)}{\lambda_{\lambda_{\alpha}}\left(t_{2}\right)-1}\right) \prod_{t_{2}+1}^{t_{1}-1} \frac{\lambda_{\alpha}}{\lambda_{\beta}}+\widehat{r}_{\alpha \beta}\left(t_{1}-1\right) \frac{\lambda_{\alpha}}{\lambda_{\beta}}\left(t_{1}-1\right)+\widehat{r}_{\alpha \beta}\left(t_{1}\right)\right] \widehat{r}_{\beta \nu}\left(t_{1}\right) \mid .
\end{aligned}
$$

Similar to (2.4.13), we have

$$
\left|\frac{\widehat{r}_{\alpha \beta}\left(t_{1}-1\right)}{\frac{\lambda_{\beta}}{\lambda_{\alpha}}\left(t_{1}-1\right)-1}\right| \leq \sum_{t_{2}=t_{1}-1}^{\infty}\left|\triangle_{t_{2}} \frac{\widehat{r}_{\alpha \beta}\left(t_{2}\right)}{\frac{\lambda_{\beta}}{\lambda_{\alpha}}\left(t_{2}\right)-1}\right| .
$$

As $\left|\frac{\lambda_{\alpha}}{\lambda_{\beta}}\left(t_{1}-1\right)\right|$ is bounded by $K_{1}$, the assumptions $(2.4 .3),(2.4 .4)$ and $(2.4 .5)$ guarantee that (2.3.2) tends to 0.

Case IV. $(\alpha, \beta) \in(1.2 .11)$, and $(\alpha, \gamma) \in(1.2 .11)$. Now the summation in (2.3.2) is

$$
\begin{aligned}
& \sum_{t_{1}=a}^{t-1}\left|\left[\sum_{t_{2}=a}^{t_{1}-1} \widehat{r}_{\alpha \beta}\left(t_{2}\right) \prod_{t_{2}}^{t_{1}-1} \frac{\lambda_{\alpha}}{\lambda_{\beta}}+\widehat{r}_{\alpha \beta}\left(t_{1}\right)\right] \widehat{r}_{\beta \nu}\left(t_{1}\right) \prod_{t_{1}}^{t-1} \frac{\lambda_{\alpha}}{\lambda_{\gamma}}\right| \\
= & \sum_{t_{1}=a}^{t-1} \mid\left[\frac{\widehat{r}_{\alpha \beta}\left(t_{1}-1\right)}{\frac{\lambda_{\beta}}{\lambda_{\alpha}}\left(t_{1}-1\right)-1} \cdot \frac{\lambda_{\alpha}}{\lambda_{\beta}}\left(t_{1}-1\right)-\frac{\widehat{r}_{\alpha \beta}(a)}{\frac{\lambda_{\beta}}{\lambda_{\alpha}}(a)-1} \prod_{a}^{t_{1}-1} \frac{\lambda_{\alpha}}{\lambda_{\beta}}\right. \\
& \left.-\sum_{t_{2}=a}^{t_{1}-2}\left(\triangle_{t_{2}} \frac{\widehat{r}_{\alpha \beta}\left(t_{2}\right)}{\frac{\lambda_{\beta}}{\lambda_{\alpha}}\left(t_{2}\right)-1}\right) \prod_{t_{2}+1}^{t_{1}-1} \frac{\lambda_{\alpha}}{\lambda_{\beta}}+\widehat{r}_{\alpha \beta}\left(t_{1}-1\right) \frac{\lambda_{\alpha}}{\lambda_{\beta}}\left(t_{1}-1\right)+\widehat{r}_{\alpha \beta}\left(t_{1}\right)\right] \widehat{r}_{\beta \nu}\left(t_{1}\right) \prod_{t_{1}}^{t-1} \frac{\lambda_{\alpha}}{\lambda_{\gamma}} \mid .
\end{aligned}
$$

By Lemma 2.3.2 and the same process in Case III, we get that the summation in (2.3.2) tends to 0 .

REMARK. (i) If $\frac{\widehat{r}_{\alpha \beta}(t)}{\frac{\lambda_{\beta}}{\lambda_{\alpha}}(t)-1}$ is monotone then (2.4.1) implies (2.4.2) and (2.4.3) may be written as $\frac{\widehat{r}_{\alpha \beta}(t-1)}{\frac{\lambda_{\beta}}{\lambda_{\alpha}}(t-1)-1} \widehat{r}_{\beta \nu}(t) \in l^{1}$.

(ii) If $\lambda_{\alpha}=\lambda_{\beta}$ the theorem holds provided that $\widehat{r}_{\alpha \beta} \equiv 0$. In this case (2.4.4) and (2.4.5) are irrelevant.

(iii) Conditions (2.4.3)-(2.4.5) are not too severe requirements since in each of them $\widehat{r}_{\beta \nu}(t)$ is multiplied by a factor which tends to zero. 


\subsection{Some examples and comparisons}

The following examples are designed to bring out some manners that Theorem 2.4.1 could be applied. Consider Eq. (2.1.2) with

$$
D(t)=\operatorname{diag}\left\{c_{i} t_{\underline{\underline{p_{i}}}}\right\}, \quad R(t)=\left\{a_{i j} t_{\underline{q_{i j}}}^{\underline{2}}\right\}, a_{i i}=0, i, j=1,2, \ldots, n .
$$

Now let us check when do the assumption (2.4.1)-(2.4.5) hold. The first part of (2.4.1) means

$$
\frac{\widehat{r}_{\alpha \beta}(t)}{\frac{\lambda_{\beta}}{\lambda_{\alpha}}(t)-1}=\frac{\frac{r_{\alpha \beta}(t)}{\lambda_{\alpha}}}{\frac{\lambda_{\beta}}{\lambda_{\alpha}}(t)-1}=\frac{r_{\alpha \beta}(t)}{\lambda_{\beta}(t)-\lambda_{\alpha}(t)}=\frac{a_{\alpha \beta} t^{q_{\alpha \beta}}}{c_{\beta} t^{\underline{\underline{\beta}}}-c_{\alpha} t_{\underline{\underline{p_{\alpha}}}}}=O\left(t^{q_{\alpha \beta}-\max \left(p_{\alpha}, p_{\beta}\right)}\right) .
$$

Therefore, the first part of (2.4.1) holds when

$$
q_{\alpha \beta}<\max \left(p_{\alpha}, p_{\beta}\right) .
$$

Since $\widehat{r}_{\alpha \beta}(t)=\frac{r_{\alpha \beta}(t)}{\lambda_{\alpha}}=O\left(t^{q_{\alpha \beta}-p_{\alpha}}\right)$, the second part of (2.4.1) holds when

$$
q_{\alpha \beta}<p_{\alpha} .
$$

The relation (2.4.2) leads to the same condition (2.5.3). Therefore, condition (2.4.1) and (2.4.2) hold when (2.5.4) is valid.

Since $\widehat{r}_{\beta \nu}(t)=\frac{r_{\beta \nu}(t)}{\lambda_{\beta}}=O\left(t^{q_{\beta \nu}-p_{\beta}}\right)$, condition (2.4.3) becomes

$$
q_{\alpha \beta}+q_{\beta \nu}-p_{\beta}-\max \left(p_{\alpha}, p_{\beta}\right)<-1 .
$$

(2.4.4) and (2.4.5) are assumed only when $(\alpha, \beta)$ satisfies (1.2.11), which means $\prod_{a}^{t}\left|\frac{\lambda_{\alpha}\left(t_{1}\right)}{\lambda_{\beta}\left(t_{1}\right)}\right| \rightarrow$ 0 . At this time

$$
\prod_{a}^{t}\left|\frac{\lambda_{\alpha}\left(t_{1}\right)}{\lambda_{\beta}\left(t_{1}\right)}\right|=\prod_{a}^{t}\left|\frac{c_{\alpha}}{c_{\beta}}\right| \cdot\left|\frac{t_{1}^{\frac{p_{\alpha}}{1}}}{t_{\frac{p_{\beta}}{1}}}\right| \sim \prod_{a}^{t}\left|\frac{c_{\alpha}}{c_{\beta}}\right| \cdot\left|t_{1}^{p_{\alpha}-p_{\beta}}\right| \rightarrow 0
$$

as $t \rightarrow \infty$ may happen when

$$
p_{\alpha}<p_{\beta}
$$

or

$$
p_{\alpha}=p_{\beta} \text { and }\left|c_{\alpha}\right|<\left|c_{\beta}\right| .
$$

(i) If $p_{\alpha}<p_{\beta}$, let $K$ be the number in $\{a, a+1, a+2, \ldots\}$ such that

$$
\left|\frac{c_{\alpha}}{c_{\beta}}\right| \cdot\left|K^{p_{\alpha}-p_{\beta}}\right| \geq 1 \text { and }\left|\frac{c_{\alpha}}{c_{\beta}}\right| \cdot\left|(K+1)^{p_{\alpha}-p_{\beta}}\right|<1 .
$$

Then, there exists some $\delta \in(0,1)$ such that when $t>K$,

$$
\prod_{a}^{t}\left|\frac{c_{\alpha}}{c_{\beta}}\right| \cdot\left|t_{1}^{p_{\alpha}-p_{\beta}}\right|=\left(\prod_{a}^{K}\left|\frac{c_{\alpha}}{c_{\beta}}\right| \cdot\left|t_{1}^{p_{\alpha}-p_{\beta}}\right|\right) \cdot\left(\prod_{K+1}^{t}\left|\frac{c_{\alpha}}{c_{\beta}}\right| \cdot\left|t_{1}^{p_{\alpha}-p_{\beta}}\right|\right) \leq M_{0}(a) \delta^{t},
$$

where $M_{0}(s)=\left(\prod_{s}^{K}\left|\frac{c_{\alpha}}{c_{\beta}}\right| \cdot\left|t_{1}^{p_{\alpha}-p_{\beta}}\right|\right) \delta^{-K}$ with the proposition $M_{0}(a)>M_{0}(a+1)>M(a+$ 2) $\cdots$

Therefore, the first part of (2.4.4), $\widehat{r}_{\beta \nu}(t) \prod_{a}^{t} \frac{\lambda_{\alpha}}{\lambda_{\beta}} \in l^{1}$, is obviously by the fast decay of $\delta^{t}$. The last two terms of (2.4.4), $\widehat{r}_{\alpha \beta}(t-1) \widehat{r}_{\beta \nu}(t) \in l^{1}$ and $\widehat{r}_{\alpha \beta}(t) \widehat{r}_{\beta \nu}(t) \in l^{1}$, lead to the condition 


$$
q_{\alpha \beta}-p_{\alpha}+q_{\beta \nu}-p_{\beta}<-1 \text {. }
$$

We can verify that the conditions (2.5.5) and (2.5.9) with $p_{\alpha}<p_{\beta}$ is equivalent to (2.5.9) for any $p_{\alpha}$ and $p_{\beta}$.

Since $\triangle_{t_{2}} \frac{\widehat{r}_{\alpha \beta}\left(t_{2}\right)}{\frac{\lambda \beta}{\lambda \alpha}\left(t_{2}\right)-1} \in l^{1}$ and $\left|\prod_{t_{2}+1}^{t} \frac{\lambda_{\alpha}}{\lambda_{\beta}}\right| \leq M_{0}\left(t_{2}+1\right) \delta^{t} \leq M_{0}(a) \delta^{t},(2.4 .5)$ is bounded by

$$
\left|\widehat{r}_{\beta \nu}(t) \sum_{t_{2}=a}^{t-1}\left[\left(\triangle_{t_{2}} \frac{\widehat{r}_{\alpha \beta}\left(t_{2}\right)}{\frac{\lambda_{\beta}}{\lambda_{\alpha}}\left(t_{2}\right)-1}\right) \prod_{t_{2}+1}^{t} \frac{\lambda_{\alpha}}{\lambda_{\beta}}\right]\right| \leq\left|\widehat{r}_{\beta \nu}(t)\right|\left(\sum_{t_{2}=a}^{t-1}\left|\triangle_{t_{2}} \frac{\widehat{r}_{\alpha \beta}\left(t_{2}\right)}{\frac{\lambda_{\beta}}{\lambda_{\alpha}}\left(t_{2}\right)-1}\right|\right) M_{0}(a) \delta^{t} \in l^{1}
$$

because the fast decay of $\delta^{t}$.

(ii) If $p_{\alpha}=p_{\beta}$ and $\left|c_{\alpha}\right|<\left|c_{\beta}\right|$, then

$$
\left|\prod_{a}^{t} \frac{\lambda_{\alpha}}{\lambda_{\beta}}\right|=\left|\frac{c_{\alpha}}{c_{\beta}}\right|^{t-a}
$$

$\widehat{r}_{\beta \nu}(t) \prod_{a}^{t} \frac{\lambda_{\alpha}}{\lambda_{\beta}} \in l^{1}$ is valid because of the fast decay of $\left|\frac{c_{\alpha}}{c_{\beta}}\right|^{t-a}$. Similarly as in (i), The second and third terms of (2.4.4) lead to the same condition of (2.5.9).

We now consider the condition (2.4.5). By Lemma 2.3.2, (2.4.2) and (2.5.10), we get that

$$
\sum_{t_{2}=a}^{t-1}\left[\left(\triangle_{t_{2}} \frac{\widehat{r}_{\alpha \beta}\left(t_{2}\right)}{\frac{\lambda_{\beta}}{\lambda_{\alpha}}\left(t_{2}\right)-1}\right) \prod_{t_{2}+1}^{t} \frac{\lambda_{\alpha}}{\lambda_{\beta}}\right] \rightarrow 0, \text { as } t \rightarrow \infty .
$$

Hence, if $\widehat{r}_{\beta \nu}(t) \in l^{1}$, which is guaranteed by $q_{\beta \nu}-p_{\beta}<-1$, condition (2.4.5) is valid.

In conclusion, when (1) $q_{\alpha \beta}<p_{\alpha}$, (2) $q_{\alpha \beta}-p_{\alpha}+q_{\beta \nu}-p_{\beta}<-1$, and (3) $q_{\beta \nu}-p_{\beta}<-1$ in case $p_{\alpha}=p_{\beta}$ and $\left|c_{\alpha}\right|<\left|c_{\beta}\right|$, the difference system (2.1.2) with $D(t)$ and $R(t)$ defined in (2.5.1) is left almost diagonal.

Consider, for example, the system

$$
Y(t+1)=\left[\left(\begin{array}{cc}
t^{\underline{1}} & 0 \\
0 & t^{\underline{3}}
\end{array}\right)+\left(\begin{array}{cc}
0 & t^{\underline{0.9}} \\
t^{\underline{\underline{x}}} & 0
\end{array}\right)\right] Y(t), \quad 1 \leq t \leq \infty .
$$

The analog of Levinson's Theorem (Theorem 1.2.4) cannot be applied to system (2.5.11) because $\hat{r}_{12}=\frac{r_{12}}{\lambda_{1}}=\frac{t^{0.9}}{t^{1}}=O\left(t^{-0.1}\right)$ that is not belong to $l^{1}$. Neither can Proposition 2.2 in [16] be applied because of the same reason. However, we can get the conclusion that (2.5.11) is left almost diagonal by our method easily since it fits (2.5.4) and (2.5.9). 


\section{CHAPTER 3}

\section{Right almost diagonal differential equations}

\subsection{Introduction}

We consider the differential system

$$
Y^{\prime}(t)=[D(t)+R(t)] Y(t)
$$

with

$$
\begin{aligned}
D(t) & =\operatorname{diag}\left\{\lambda_{1}(t), \lambda_{2}(t), \ldots, \lambda_{n}(t)\right\}, \\
R(t) & =\left(r_{j k}(t)\right)_{j, k=1}^{n}, \quad \text { where } r_{k k}(t)=0 .
\end{aligned}
$$

The main goal of this chapter is to present a new technique and a new set of conditions on "potentially oscillatory" differential equations that will render (3.1.1) a right almost diagonal system, i.e., (3.1.1) has a fundamental solution in the form

$$
Y(t)=\exp \left(\int_{t_{0}}^{t} D(s) d s\right)(I+P(t)), t_{0} \in[a, \infty),
$$

where $P(t) \rightarrow 0$ as $t \rightarrow \infty$. Here, a "potentially oscillatory" differential system, which is also defined in Definition 1.1.2, means that

$$
\left|\operatorname{Re} \int_{t_{1}}^{t_{2}}\left(\lambda_{j}(s)-\lambda_{k}(s)\right) d s\right| \leq M
$$

for all $a \leq t_{1}, t_{2} \leq \infty$ where $M$ is a fixed positive number.

Our technique involves the conversion of a differential equation for $P(t)$ into a family of integral equations in a manner to be detailed in Section 3.2. Each member of this family points to a different theorem of asymptotic integration. Conditions will be given which will guarantee that the system (3.1.1) is right almost diagonal. Examples will be discussed in Section 3.3, that are amenable to our theorem and to which the results of $[\mathbf{9}, \mathbf{1 2}, \mathbf{2 2}, \mathbf{2 3}, \mathbf{3 2}]$ do not apply.

\subsection{Asymptotic integration}

It can be easily verified that a fundamental solution $Y(t)$ of (3.1.1) can be written as

$$
Y(t)=\Phi(t) Z(t)
$$

where,

$$
\Phi(t)=\exp \left(\int_{t_{0}}^{t} D(s) d s\right)
$$

is a fundamental solution of

$$
\Phi^{\prime}(t)=D(t) \Phi(t)
$$


and $Z(t)$ is a fundamental solution of

$$
Z^{\prime}(t)=\tilde{R}(t) Z(t)
$$

with

$$
\tilde{R}(t)=\Phi^{-1}(t) R(t) \Phi(t) .
$$

Let $Z(t) \in C[a, b), a<b \leq \infty$, be an $n \times n$ matrix function. Define a linear operator $\mathcal{L}$ by

$$
(\mathcal{L} Z)(t):=\int_{b}^{t} \tilde{R}(s) Z(s) d s .
$$

provided that the integral exists for $t \in[a, b)$. It is evident that $Z(t)$ is a fundamental solution of $Z^{\prime}(t)=\tilde{R}(t) Z(t)$ if $Z(t)$ satisfies the integral equation

$$
Z(t)=I+\mathcal{L} Z(t) .
$$

Define for $m$ an integer and $m \geq 2$, the matrix function $C_{1}(s, t)$ by

$$
C_{1}(s, t)=\int_{b}^{s} \int_{t_{1}}^{t} \cdots \int_{t_{m-3}}^{t} \int_{t_{m-2}}^{t} \tilde{R}\left(t_{m-1}\right) \cdots \tilde{R}\left(t_{1}\right) d t_{m-1} d t_{m-2} \cdots d t_{2} d t_{1} .
$$

A main result of this chapter is the following.

THEOREM 3.2.1. Assume that for some $m \geq 2$ we have uniformly for $b \in(a, \infty]$ that in (3.2.6) and (3.2.8)

$$
\text { (i) } \mathcal{L}^{k} I, k=1,2, \cdots, m-1 \text {, are convergent integrals for } t \in[a, b),
$$$$
\text { (but not necessary absolutely convergent integrals) }
$$$$
\text { (ii) } \quad \mathcal{L}^{k} I \rightarrow 0 \text { as } t \rightarrow b, k=1,2, \cdots, m-1, \quad \text { and }
$$$$
\text { (iii) } \int_{t}^{b}\left\|C_{1}(s, t) \tilde{R}(s)\right\| d s \rightarrow 0 \text { as } t \rightarrow b .
$$

Then, there exists a constant a large enough such that for $t \in[a, b)$, the resolvent series $\sum_{j=1}^{\infty} \mathcal{L}^{j} I$ is absolutely and uniformly convergent. Moreover, the system (3.1.1) is right almost diagonal. Namely, a fundamental solution $Y(t)$ of (3.1.1) is given by

$$
Y(t)=\exp \left(\int_{t_{0}}^{t} D(s) d s\right)(I+P(t))
$$

with $P(t)=\sum_{j=1}^{\infty} \mathcal{L}^{j} I \rightarrow 0$ as $t \rightarrow$ b.

We need first some preparatory lemmas. The first lemma below, which is an extension of Fubini's Theorem, is about changing the order of integration in certain expressions.

Lemma 3.2.2. Suppose $f: \mathbb{R}^{m} \mapsto \mathbb{R}$ with $m \geq 2$, is an integrable function, then

$$
\begin{aligned}
& \int_{b}^{t} \int_{b}^{t_{m-1}} \int_{b}^{t_{m-2}} \cdots \int_{b}^{t_{2}} \int_{b}^{t_{1}} f\left(s, t_{1}, \cdots, t_{m-1}\right) d s d t_{1} \cdots d t_{m-3} d t_{m-2} d t_{m-1} \\
= & \int_{b}^{t} \int_{s}^{t} \int_{t_{1}}^{t} \cdots \int_{t_{m-3}}^{t} \int_{t_{m-2}}^{t} f\left(s, t_{1}, \cdots, t_{m-1}\right) d t_{m-1} d t_{m-2} \cdots d t_{2} d t_{1} d s .
\end{aligned}
$$


Proof. By induction, when $m=2$, we observe that

$$
\int_{b}^{t} \int_{b}^{t_{1}} f\left(s, t_{1}\right) d s d t_{1}=\int_{b}^{t} \int_{s}^{t} f\left(s, t_{1}\right) d t_{1} d s
$$

Assume that (3.2.13) is valid for $2 \leq m \leq k$. When $m=k+1$, let $J(t)$ be the left hand side of (3.2.13) that is

$$
J(t)=\int_{b}^{t} \int_{b}^{t_{k}}\left(\int_{b}^{t_{k-1}} \cdots \int_{b}^{t_{2}} \int_{b}^{t_{1}} f\left(s, t_{1}, \cdots, t_{k}\right) d s d t_{1} \cdots d t_{k-2}\right) d t_{k-1} d t_{k} .
$$

Let $g\left(t_{k-1}, t_{k}\right)=\int_{b}^{t_{k-1}} \cdots \int_{b}^{t_{1}} f\left(s, t_{1}, \cdots, t_{k}\right) d s \cdots d t_{k-2}$, then

$$
\begin{aligned}
J(t) & =\int_{b}^{t} \int_{b}^{t_{k}} g\left(t_{k-1}, t_{k}\right) d t_{k-1} d t_{k}=\int_{b}^{t} \int_{t_{k-1}}^{t} g\left(t_{k-1}, t_{k}\right) d t_{k} d t_{k-1} \\
& =\int_{b}^{t}\left(\int_{t_{k-1}}^{t} \int_{b}^{t_{k-1}} \cdots \int_{b}^{t_{1}} f\left(s, t_{1}, \cdots, t_{k}\right) d s \cdots d t_{k-2} d t_{k}\right) d t_{k-1} .
\end{aligned}
$$

Since $t_{k-1}$ is temporarily fixed for $\int_{t_{k-1}}^{t} \int_{b}^{t_{k-1}} \cdots \int_{b}^{t_{1}} f\left(s, t_{1}, \cdots, t_{k}\right) d s \cdots d t_{k-2} d t_{k}$ in (3.2.15), we have

$$
\begin{aligned}
J(t) & =\int_{b}^{t} \int_{b}^{t_{k-1}} \int_{t_{k-1}}^{t} \int_{b}^{t_{k-2}} \cdots \int_{b}^{t_{1}} f\left(s, t_{1}, \cdots, t_{k}\right) d s \cdots d t_{k-3} d t_{k} d t_{k-2} d t_{k-1} \\
& =\int_{b}^{t} \int_{b}^{t_{k-1}} \int_{b}^{t_{k-2}} \int_{t_{k-1}}^{t} \cdots \int_{b}^{t_{1}} f\left(s, t_{1}, \cdots, t_{k}\right) d s \cdots d t_{k} d t_{k-3} d t_{k-2} d t_{k-1} \\
& =\int_{b}^{t} \int_{b}^{t_{k-1}} \int_{b}^{t_{k-2}} \cdots \int_{b}^{t_{1}} \int_{t_{k-1}}^{t} f\left(s, t_{1}, \cdots, t_{k}\right) d t_{k} d s \cdots d t_{k-3} d t_{k-2} d t_{k-1} \\
& =\int_{b}^{t} \int_{b}^{t_{k-1}} \int_{b}^{t_{k-2}} \cdots \int_{b}^{t_{1}} h\left(s, t_{1}, \cdots, t_{k-1}\right) d s \cdots d t_{k-3} d t_{k-2} d t_{k-1}
\end{aligned}
$$

in which $h\left(s, t_{1}, \cdots, t_{k-1}\right)=\int_{t_{k-1}}^{t} f\left(s, t_{1}, \cdots, t_{k}\right) d t_{k}$.

By the assumption, (3.2.13) is valid for $m=k$, thus we get

$$
\begin{aligned}
& \int_{b}^{t} \int_{b}^{t_{k-1}} \int_{b}^{t_{k-2}} \cdots \int_{b}^{t_{1}} h\left(s, t_{1}, \cdots, t_{k-1}\right) d s \cdots d t_{k-3} d t_{k-2} d t_{k-1} \\
= & \int_{b}^{t} \int_{s}^{t} \int_{t_{1}}^{t} \cdots \int_{t_{k-2}}^{t} h\left(s, t_{1}, \cdots, t_{k-1}\right) d t_{k-1} \cdots d t_{2} d t_{1} d s \\
= & \int_{b}^{t} \int_{s}^{t} \int_{t_{1}}^{t} \cdots \int_{t_{k-2}}^{t} \int_{t_{k-1}}^{t} f\left(s, t_{1}, \cdots, t_{k}\right) d t_{k} d t_{k-1} \cdots d t_{2} d t_{1} d s .
\end{aligned}
$$

Therefore, we conclude that (2.3.1) is valid for any $m \in \mathbb{N}$ with $m \geq 2$.

We then need to embed the differential system (3.1.1) in a wider family of differential equations. Consider the differential system

$$
\frac{\partial}{\partial t} Y(t, \epsilon)=(D(t)+\epsilon R(t)) Y(t, \epsilon)
$$

in which $\epsilon \in D_{\rho}$ where $D_{\rho}=\{\epsilon|| \epsilon \mid<\rho\}$, and $\rho$ is a constant satisfying $\rho>1$. Equation (3.1.1) is then a special case of (3.2.16) with $\epsilon=1$. 
Consider the corresponding integral equation

$$
\hat{Z}(t, \epsilon)=I+\int_{b}^{t} \epsilon \tilde{R}(s) \hat{Z}(s, \epsilon) d s=I+\epsilon \mathcal{L} \hat{Z}(t, \epsilon),
$$

where we extend the definition of $\mathcal{L}$ in $(3.2 .6)$ as

$$
\mathcal{L} \hat{Z}(t, \epsilon)=\int_{b}^{t} \tilde{R}(s) \hat{Z}(s, \epsilon) d s .
$$

If $\hat{Z}(t, \epsilon)$ is a solution of $\hat{Z}=I+\epsilon \mathcal{L} \hat{Z}$, then

$$
\hat{Z}=I+\epsilon \mathcal{L} \hat{Z}=I+\epsilon \mathcal{L}(I+\epsilon \mathcal{L} \hat{Z})=I+\epsilon \mathcal{L} I+\cdots+\epsilon^{m-1} \mathcal{L}^{m-1} I+\epsilon^{m} \mathcal{L}^{m} \hat{Z}
$$

where $m \in \mathbb{N}$ and $m \geq 2$.

It can be easily verified that for any $m \in \mathbb{N}$ and $m \geq 2$,

$$
\mathcal{L}^{m} \hat{Z}(t, \epsilon)=\int_{b}^{t} \int_{b}^{t_{m-1}} \cdots \int_{b}^{t_{2}} \int_{b}^{t_{1}} \tilde{R}\left(t_{m-1}\right) \cdots \tilde{R}\left(t_{1}\right) \tilde{R}(s) \hat{Z}(s, \epsilon) d s d t_{1} \cdots d t_{m-2} d t_{m-1} .
$$

By Lemma 3.2.2 we have

$$
\begin{aligned}
& \mathcal{L}^{m} \hat{Z}(t, \epsilon) \\
= & \int_{b}^{t} \int_{s}^{t} \int_{t_{1}}^{t} \cdots \int_{t_{m-3}}^{t} \int_{t_{m-2}}^{t} \tilde{R}\left(t_{m-1}\right) \cdots \tilde{R}\left(t_{1}\right) \tilde{R}(s) \hat{Z}(s, \epsilon) d t_{m-1} d t_{m-2} \cdots d t_{2} d t_{1} d s \\
= & \int_{b}^{t}\left(\int_{s}^{t} \int_{t_{1}}^{t} \cdots \int_{t_{m-3}}^{t} \int_{t_{m-2}}^{t} \tilde{R}\left(t_{m-1}\right) \cdots \tilde{R}\left(t_{1}\right) d t_{m-1} d t_{m-2} \cdots d t_{2} d t_{1}\right) \tilde{R}(s) \hat{Z}(s, \epsilon) d s \\
= & \int_{b}^{t}\left(\int_{b}^{t} \int_{t_{1}}^{t} \cdots \int_{t_{m-3}}^{t} \int_{t_{m-2}}^{t} \tilde{R}\left(t_{m-1}\right) \cdots \tilde{R}\left(t_{1}\right) d t_{m-1} d t_{m-2} \cdots d t_{2} d t_{1}\right. \\
& \left.-\int_{b}^{s} \int_{t_{1}}^{t} \cdots \int_{t_{m-3}}^{t} \int_{t_{m-2}}^{t} \tilde{R}\left(t_{m-1}\right) \cdots \tilde{R}\left(t_{1}\right) d t_{m-1} d t_{m-2} \cdots d t_{2} d t_{1}\right) \tilde{R}(s) \hat{Z}(s, \epsilon) d s \\
(3.2 .20)= & \int_{b}^{t}\left[C_{1}(t, t)-C_{1}(s, t)\right] \tilde{R}(s) \hat{Z}(s, \epsilon) d s,
\end{aligned}
$$

where $C_{1}(s, t)$ is defined in (3.2.8).

We can see that

$$
C_{1}(t, t)=\int_{b}^{t} \cdots \int_{t_{m-3}}^{t} \int_{t_{m-2}}^{t} \tilde{R}\left(t_{m-1}\right) \cdots \tilde{R}\left(t_{1}\right) d t_{m-1} d t_{m-2} \cdots d t_{1}=\mathcal{L}^{m-1} I .
$$

Therefore, (3.2.20) implies

$$
\mathcal{L}^{m} \hat{Z}(t, \epsilon)=\mathcal{L}^{m-1} I \cdot \mathcal{L} \hat{Z}(t, \epsilon)-\int_{b}^{t} C_{1}(s, t) \tilde{R}(s) \hat{Z}(s, \epsilon) d s .
$$

Here we get a useful identity for a later discussion,

$$
-\int_{b}^{t} C_{1}(s, t) \tilde{R}(s) \hat{Z}(s, \epsilon) d s \equiv \mathcal{L}^{m} \hat{Z}(t, \epsilon)-\mathcal{L}^{m-1} I \cdot \mathcal{L} \hat{Z}(t, \epsilon) .
$$


From (3.2.17), (3.2.19) and (3.2.22), we get

$$
\begin{aligned}
\hat{Z} & =I+\epsilon \mathcal{L} I+\cdots+\epsilon^{m-1} \mathcal{L}^{m-1} I+\epsilon^{m}\left(\mathcal{L}^{m-1} I \cdot \mathcal{L} \hat{Z}-\int_{b}^{t} C_{1}(s, t) \tilde{R}(s) \hat{Z}(s, \epsilon) d s\right) \\
& =I+\epsilon \mathcal{L} I+\cdots+\epsilon^{m-1} \mathcal{L}^{m-1} I+\epsilon^{m}\left(\left(\mathcal{L}^{m-1} I\right) \frac{\hat{Z}-I}{\epsilon}-\int_{b}^{t} C_{1}(s, t) \tilde{R}(s) \hat{Z}(s, \epsilon) d s\right) \\
& =I+\epsilon \mathcal{L} I+\cdots+\epsilon^{m-2} \mathcal{L}^{m-2} I+\epsilon^{m-1}\left(\mathcal{L}^{m-1} I\right) \hat{Z}-\epsilon^{m} \int_{b}^{t} C_{1}(s, t) \tilde{R}(s) \hat{Z}(s, \epsilon) d s
\end{aligned}
$$

which leads to

$$
\left(I-\epsilon^{m-1} \mathcal{L}^{m-1} I\right) \hat{Z}=I+\epsilon \mathcal{L} I+\cdots+\epsilon^{m-2} \mathcal{L}^{m-2} I-\epsilon^{m} \int_{b}^{t} C_{1}(s, t) \tilde{R}(s) \hat{Z}(s, \epsilon) d s .
$$

Thus we obtain a new equation

$$
\hat{Z}(t, \epsilon)=\left(I-\epsilon^{m-1} \mathcal{L}^{m-1} I\right)^{-1}\left[\sum_{\nu=0}^{m-2} \epsilon^{\nu} \mathcal{L}^{\nu} I-\epsilon^{m} \int_{b}^{t} C_{1}(s, t) \tilde{R}(s) \hat{Z}(s, \epsilon) d s\right],
$$

provided that $\left(I-\epsilon^{m-1} \mathcal{L}^{m-1} I\right)^{-1}$ exists.

Equation (3.2.24) can be rewritten as

$$
\hat{Z}=\tilde{H}+\tilde{\mathcal{L}} \hat{Z}
$$

where

$$
\begin{aligned}
& \tilde{H}:=\left(I-\epsilon^{m-1} \mathcal{L}^{m-1} I\right)^{-1} \sum_{\nu=0}^{m-2} \epsilon^{\nu} \mathcal{L}^{\nu} I \\
& \tilde{\mathcal{L}} \hat{Z}(t, \epsilon):=-\left(I-\epsilon^{m-1} \mathcal{L}^{m-1} I\right)^{-1} \epsilon^{m} \int_{b}^{t} C_{1}(s, t) \tilde{R}(s) \hat{Z}(s, \epsilon) d s .
\end{aligned}
$$

For the convenience of the later discussion, we define

$$
\mathcal{L}^{0} I=I, \quad \tilde{\mathcal{L}}^{0} I=\tilde{H} .
$$

For any bounded valued matrix function $A(t, \epsilon)$, we define

$$
\|A(t, \epsilon)\|=\sum_{i, j=1}^{n}\left|a_{i j}(t, \epsilon)\right|
$$

which leads of course to the useful relation

$$
\|A(t, \epsilon) B(t, \epsilon)\| \leq\|A(t, \epsilon)\| \cdot\|B(t, \epsilon)\| .
$$

Then we have the following lemma.

LEMMA 3.2.3. Assume that we have uniformly for $b \in(a, \infty]$,

(i) $\mathcal{L}^{m-1} I$ is a convergent integral on $[a, b)$

(ii) $\mathcal{L}^{m-1} I \rightarrow 0$ as $t \rightarrow b$, and

(iii) $\int_{t}^{b}\left\|C_{1}(s, t) \tilde{R}(s)\right\| d s \rightarrow 0$ as $t \rightarrow b$.

Then, for $\epsilon \in D_{\rho}$ with $\rho>1$,

(a) $\hat{X}(t, \epsilon)=\sum_{k=0}^{\infty} \tilde{\mathcal{L}}^{k} \tilde{H}$ converges absolutely and uniformly when $t$ belongs to some interval 
$[a, b)$ and $a$ is large enough. $\hat{X}(t, \epsilon)$ is the unique solution of (3.2.25) for $t \in[a, b)$. Moreover, (b) $\frac{\partial}{\partial t} \hat{X}(t, \epsilon)=\epsilon \tilde{R}(t) \hat{X}(t, \epsilon)$.

Proof. (a) Since $\mathcal{L}^{m-1} I \rightarrow 0, \int_{t}^{b}\left\|C_{1}(s, t) \tilde{R}(s)\right\| d s \rightarrow 0$ as $t \rightarrow b$, and $\epsilon \in D_{\rho}$, there exists some $a \in \mathbb{R}$ and $\delta \in(0,1)$ such that for any $t \in[a, b),\left(I-\epsilon^{m-1} \mathcal{L}^{m-1} I\right)^{-1}$ exists and

$$
\left\|\left(I-\epsilon^{m-1} \mathcal{L}^{m-1} I\right)^{-1}\right\| \cdot|\epsilon|^{m} \int_{t}^{b}\left\|C_{1}(s, t) \tilde{R}(s)\right\| d s<\delta .
$$

For each fixed $\epsilon \in D_{\rho}$, define when $t \in[a, b)$,

$$
\|\mid A(t, \epsilon)\|=\sup _{t \in[a, b)}\|A(t, \epsilon)\| .
$$

Define a series $\left\{\hat{Z}_{n}\right\}_{n=0,1,2 \ldots}$ by

$$
\hat{Z}_{0}=\tilde{H}, \quad \hat{Z}_{j+1}=\tilde{H}+\tilde{\mathcal{L}} \hat{Z}_{j}, \text { for } j=0,1,2, \cdots .
$$

For any $t \in[a, b), \epsilon \in D_{\rho}$, we have

$$
\begin{aligned}
& \left\|\hat{Z}_{j+1}(t, \epsilon)-\hat{Z}_{j}(t, \epsilon)\right\| \\
= & \left\|\tilde{\mathcal{L}} \hat{Z}_{j}(t, \epsilon)-\tilde{\mathcal{L}} \hat{Z}_{j-1}(t, \epsilon)\right\| \\
\leq & \left\|\left(I-\epsilon^{m-1} \mathcal{L}^{m-1} I\right)^{-1} \epsilon^{m}\right\| \int_{b}^{t}\left\|C_{1}(s, t) \tilde{R}(s)\left[\hat{Z}_{j}(s, \epsilon)-\hat{Z}_{j-1}(s, \epsilon)\right]\right\| d s \\
\leq & \left(\left\|\left(I-\epsilon^{m-1} \mathcal{L}^{m-1} I\right)^{-1}\right\| \cdot|\epsilon|^{m} \int_{t}^{b}\left\|C_{1}(s, t) \tilde{R}(s)\right\| d s\right)\left\|\hat{Z}_{j}-\hat{Z}_{j-1}\right\| \| \\
\leq & \delta\left\|\hat{Z}_{j}-\hat{Z}_{j-1} \mid\right\| .
\end{aligned}
$$

Therefore, $\left\|\hat{Z}_{j+1}-\hat{Z}_{j}\right\||| \leq \delta\left|\left\|\hat{Z}_{j}-\hat{Z}_{j-1} \mid\right\|\right.$. Thus $\left\{\hat{Z}_{n}\right\}$ converges uniformly for $\epsilon \in D_{\rho}$ and $t \in[a, b)$.

At the same time, notice

$$
\hat{Z}_{n}=\tilde{H}+\tilde{\mathcal{L}} \hat{Z}_{n-1}=\tilde{H}+\tilde{\mathcal{L}} \tilde{H}+\tilde{\mathcal{L}}^{2} \hat{Z}_{n-2}=\tilde{H}+\tilde{\mathcal{L}} \tilde{H}+\cdots+\tilde{\mathcal{L}}^{n} \tilde{H}
$$

so that

$$
\lim _{n \rightarrow \infty} \hat{Z}_{n}=\sum_{k=0}^{\infty} \tilde{\mathcal{L}}^{k} \tilde{H}
$$

We conclude that $\sum_{k=0}^{\infty} \tilde{\mathcal{L}}^{k} \tilde{H}$ converges absolutely and uniformly for $\epsilon \in D_{\rho}$ and $t \in[a, b)$.

As $\sum_{k=0}^{\infty} \tilde{\mathcal{L}}^{k} \tilde{H}=\tilde{\tilde{H}}+\tilde{\mathcal{L}}\left(\sum_{k=0}^{\infty} \tilde{\mathcal{L}}^{k} \tilde{H}\right)$, we get that $\hat{X}(t, \epsilon)=\sum_{k=0}^{\infty} \tilde{\mathcal{L}}^{k} \tilde{H}$ is a solution of $(3.2 .25)$.

Since $\|\tilde{\mathcal{L}}\| \leq\left\|\left(I-\epsilon^{m-1} \mathcal{L}^{m-1} I\right)^{-1}\right\| \cdot|\epsilon|^{m} \int_{t}^{b}\left\|C_{1}(s, t) \tilde{R}(s)\right\| d s<\delta<1$, we also have that $\hat{X}(t, \epsilon)$ is the unique solution of $(3.2 .25)$.

(b) Put

$$
\hat{Z}_{0}=\tilde{H}=\left(I-\epsilon^{m-1} \mathcal{L}^{m-1} I\right)^{-1} \sum_{\nu=0}^{m-2} \epsilon^{\nu} \mathcal{L}^{\nu} I .
$$

Then, $\hat{Z}_{0}(t, \epsilon)$ is an analytical matrix function of $\epsilon$ for $\epsilon \in D_{\rho}$, uniformly for $b \in(a, \infty]$. 
By induction, put

$$
\hat{Z}_{j+1}=\tilde{H}+\tilde{\mathcal{L}} \hat{Z}_{j}=\left(I-\epsilon^{m-1} \mathcal{L}^{m-1} I\right)^{-1}\left[\sum_{\nu=0}^{m-2} \epsilon^{\nu} \mathcal{L}^{\nu} I-\epsilon^{m} \int_{b}^{t} C_{1}(s, t) \tilde{R}(s) \hat{Z}_{j}(s, \epsilon) d s\right] .
$$

Then, each $\hat{Z}_{j}(t, \epsilon)$ is an analytical matrix function of $\epsilon$ for $\epsilon \in D_{\rho}$, uniformly for $b \in(a, \infty]$.

Now, since $\hat{Z}_{j}(t, \epsilon) \rightarrow \hat{X}(t, \epsilon)$, uniformly for $t \in[a, b), b \in(a, \infty]$ and $\epsilon \in D_{\rho}$, as $j \rightarrow \infty$, we conclude that $\hat{X}(t, \epsilon)$ is an analytical matrix function of $\epsilon$ for $\epsilon \in D_{\rho}$. Therefore, we may express $\hat{X}$ as an absolutely convergence power series of $\epsilon$ in $D_{\rho}$ with $\rho>1$. Namely,

$$
\hat{X}(t, \epsilon)=\sum_{j=0}^{\infty} \hat{A}_{j}(t) \epsilon^{j}
$$

Let us find the coefficients $\hat{A}_{j}(t)$. Since $\hat{X}(t, \epsilon)$ is a solution of equation (3.2.25), it is also a solution of

$$
\left(I-\epsilon^{m-1} \mathcal{L}^{m-1} I\right) \hat{Z}(t, \epsilon)=\sum_{\nu=0}^{m-2} \epsilon^{\nu} \mathcal{L}^{\nu} I-\epsilon^{m} \int_{b}^{t} C_{1}(s, t) \tilde{R}(s) \hat{Z}(s, \epsilon) d s .
$$

Therefore, together with the identity (3.2.23), we get,

$$
\left(I-\epsilon^{m-1} \mathcal{L}^{m-1} I\right) \hat{X}(t, \epsilon)=\sum_{\nu=0}^{m-2} \epsilon^{\nu} \mathcal{L}^{\nu} I+\epsilon^{m}\left(\mathcal{L}^{m} \hat{X}(t, \epsilon)-\mathcal{L}^{m-1} I \cdot \mathcal{L} \hat{X}(t, \epsilon)\right) .
$$

So that

$$
\begin{aligned}
\hat{X}(t, \epsilon) & =\sum_{\nu=0}^{m-2} \epsilon^{\nu} \mathcal{L}^{\nu} I+\epsilon^{m}\left(\mathcal{L}^{m} \hat{X}(t, \epsilon)-\mathcal{L}^{m-1} I \cdot \mathcal{L} \hat{X}(t, \epsilon)\right)+\epsilon^{m-1} \mathcal{L}^{m-1} I \cdot \hat{X}(t, \epsilon) \\
& =\sum_{\nu=0}^{m-2} \epsilon^{\nu} \mathcal{L}^{\nu} I+\epsilon^{m-1} \mathcal{L}^{m-1} I(\hat{X}(t, \epsilon)-\epsilon \mathcal{L} \hat{X}(t, \epsilon))+\epsilon^{m} \mathcal{L}^{m} \hat{X}(t, \epsilon)
\end{aligned}
$$

Notice that

$$
\begin{aligned}
\hat{X}(t, \epsilon) & =\sum_{j=0}^{\infty} \hat{A}_{j}(t) \epsilon^{j}=\hat{A}_{0}(t)+\sum_{j=0}^{\infty} \hat{A}_{j+1}(t) \epsilon^{j+1}, \\
\epsilon \mathcal{L} \hat{X}(t, \epsilon) & =\epsilon \mathcal{L}\left(\sum_{j=0}^{\infty} \hat{A}_{j}(t) \epsilon^{j}\right)=\sum_{j=0}^{\infty}\left(\mathcal{L} \hat{A}_{j}(t)\right) \epsilon^{j+1},
\end{aligned}
$$

we get that in $(3.2 .36)$,

$$
\begin{aligned}
& \epsilon^{m-1} \mathcal{L}^{m-1} I(\hat{X}(t, \epsilon)-\epsilon \mathcal{L} \hat{X}(t, \epsilon)) \\
= & \epsilon^{m-1} \mathcal{L}^{m-1} I\left(\hat{A}_{0}(t)+\sum_{j=0}^{\infty} \hat{A}_{j+1}(t) \epsilon^{j+1}-\sum_{j=0}^{\infty}\left(\mathcal{L} \hat{A}_{j}(t)\right) \epsilon^{j+1}\right) \\
= & \left(\mathcal{L}^{m-1} I\right) \hat{A}_{0}(t) \epsilon^{m-1}+\sum_{j=0}^{\infty}\left(\mathcal{L}^{m-1} I\right)\left(\hat{A}_{j+1}(t)-\mathcal{L} \hat{A}_{j}(t)\right) \epsilon^{m+j}
\end{aligned}
$$


At the same time,

$$
\epsilon^{m} \mathcal{L}^{m} \hat{X}(t, \epsilon)=\epsilon^{m} \mathcal{L}^{m}\left(\sum_{j=0}^{\infty} \hat{A}_{j}(t) \epsilon^{j}\right)=\sum_{j=0}^{\infty}\left(\mathcal{L}^{m} \hat{A}_{j}(t)\right) \epsilon^{m+j} .
$$

Hence, (3.2.36) becomes

$$
\begin{aligned}
\sum_{k=0}^{\infty} \hat{A}_{k}(t) \epsilon^{k}= & \sum_{\nu=0}^{m-2} \mathcal{L}^{j} I \cdot \epsilon^{j}+\left(\mathcal{L}^{m-1} I\right) \hat{A}_{0}(t) \epsilon^{m-1} \\
& +\sum_{j=0}^{\infty}\left(\mathcal{L}^{m-1} I\right)\left(\hat{A}_{j+1}(t)-\mathcal{L} \hat{A}_{j}(t)\right) \epsilon^{m+j}+\sum_{j=0}^{\infty}\left(\mathcal{L}^{m} \hat{A}_{j}(t)\right) \epsilon^{m+j} \\
= & \sum_{\nu=0}^{m-2} \mathcal{L}^{\nu} I \cdot \epsilon^{\nu}+\left(\mathcal{L}^{m-1} I\right) \hat{A}_{0}(t) \epsilon^{m-1} \\
& +\sum_{j=0}^{\infty}\left[\mathcal{L}^{m} \hat{A}_{j}(t)+\left(\mathcal{L}^{m-1} I\right)\left(\hat{A}_{j+1}(t)-\mathcal{L} \hat{A}_{j}(t)\right)\right] \epsilon^{m+j}
\end{aligned}
$$

By comparing the coefficients of $\epsilon^{0}, \epsilon^{1}, \cdots, \epsilon^{m-2}$ in both sides of (3.2.37), we get

$$
\hat{A}_{0}(t)=I, \hat{A}_{1}(t)=\mathcal{L} I, \cdots, \hat{A}_{m-2}(t)=\mathcal{L}^{m-2} I .
$$

Moreover, by comparing the coefficient of $\epsilon^{m-1}$ in (3.2.37), we get

$$
\hat{A}_{m-1}(t)=\left(\mathcal{L}^{m-1} I\right) \hat{A}_{0}(t)=\mathcal{L}^{m-1} I .
$$

Consider $\hat{A}_{m+j}(t), j=0,1, \cdots$. We have from (3.2.37) that

$$
\hat{A}_{m+j}=\mathcal{L}^{m} \hat{A}_{j}(t)+\left(\mathcal{L}^{m-1} I\right)\left(\hat{A}_{j+1}(t)-\mathcal{L} \hat{A}_{j}(t)\right) .
$$

By induction, it is easy to conclude that

$$
\hat{A}_{m+j}(t)=\mathcal{L}^{m+j} I, j=0,1, \cdots \text {. }
$$

Thus, we have that

$$
\hat{A}_{j}(t)=\mathcal{L}^{j} I \text { for } j=0,1, \cdots
$$

which means that

$$
\hat{X}(t, \epsilon)=\sum_{j=0}^{\infty}\left(\mathcal{L}^{j} I\right) \epsilon^{j} .
$$

Since $\sum_{j=0}^{\infty}\left(\mathcal{L}^{j} I\right) \epsilon^{j}=I+\epsilon \mathcal{L}\left(\sum_{j=0}^{\infty}\left(\mathcal{L}^{j} I\right) \epsilon^{j}\right)$, we get that $\hat{X}(t, \epsilon)$ is also a solution of $Z(t, \epsilon)=$ $I+\epsilon \mathcal{L} Z(t, \epsilon)$. Therefore, $\frac{\partial}{\partial t} \hat{X}(t, \epsilon)=\epsilon \tilde{R}(t) \hat{X}(t, \epsilon)$, which is the desired conclusion.

We can now conclude the proof of Theorem 3.2.1.

Proof. When $\epsilon=1$, we have in (3.2.26) and (3.2.27)

$$
\begin{aligned}
\tilde{H} & =\left(I-\mathcal{L}^{m-1} I\right)^{-1} \sum_{\nu=0}^{m-2} \mathcal{L}^{\nu} I \\
\tilde{\mathcal{L}} \hat{Z}(t) & =-\left(I-\mathcal{L}^{m-1} I\right)^{-1} \int_{b}^{t} C_{1}(s, t) \tilde{R}(s) \hat{Z}(s) d s .
\end{aligned}
$$


$>$ From Lemma 3.2.3, we know that $\hat{X}(t)=\sum_{\nu=0}^{\infty} \tilde{\mathcal{L}}^{\nu} \tilde{H}$ converges uniformly and absolutely, and $\hat{X}^{\prime}(t)=\tilde{R}(t) \hat{X}(t)$. Therefore,

$$
S(t)=\exp \left(\int_{t_{0}}^{t} D(s) d s\right) \hat{X}(t)
$$

is a fundamental solution of equation (3.1.1).

Also, we know that $\hat{X}(t)$ is a solution of $\hat{Z}=\tilde{H}+\tilde{\mathcal{L}} \hat{Z}$. So that

$$
\hat{X}=\tilde{H}+\tilde{\mathcal{L}} \hat{X}=\left(I-\mathcal{L}^{m-1} I\right)^{-1} \sum_{\nu=0}^{m-2} \mathcal{L}^{\nu} I-\left(I-\mathcal{L}^{m-1} I\right)^{-1} \int_{b}^{t} C_{1}(s, t) \tilde{R}(s) \hat{X}(s) d s .
$$

Hence,

$$
\begin{gathered}
\|\hat{X}-I\| \\
=\left\|\left(I-\mathcal{L}^{m-1} I\right)^{-1} \sum_{\nu=0}^{m-2} \mathcal{L}^{\nu} I-I-\left(I-\mathcal{L}^{m-1} I\right)^{-1} \int_{b}^{t} C_{1}(s, t) \tilde{R}(s) \hat{X}(s) d s\right\| \\
(3.2 .40) \leq\left\|\left(I-\mathcal{L}^{m-1} I\right)^{-1} \sum_{\nu=0}^{m-2} \mathcal{L}^{\nu} I-I\right\|+\left\|\left(I-\mathcal{L}^{m-1} I\right)^{-1}\right\| \cdot \int_{t}^{b}\left\|C_{1}(s, t) \tilde{R}(s)\right\| d s \cdot\|\hat{X}\| \|
\end{gathered}
$$

Since $\mathcal{L}^{m-1} I \rightarrow 0$, we get $\left(I-\mathcal{L}^{m-1} I\right)^{-1} \rightarrow I$ as $t \rightarrow b$. Also, by the condition (3.2.10) of Theorem 3.2.1, we have $\sum_{\nu=0}^{m-2} \mathcal{L}^{\nu} I \rightarrow I$ as $t \rightarrow b$. Therefore,

$$
\left\|\left(I-\mathcal{L}^{m-1} I\right)^{-1} \sum_{\nu=0}^{m-2} \mathcal{L}^{\nu} I-I\right\| \rightarrow 0 \text { as } t \rightarrow b .
$$

$\|\hat{X}\| \|$ is finite because $\sum_{k=0}^{\infty} \tilde{\mathcal{L}}^{k} \tilde{H}$ converges uniformly on $[a, b) .\left\|\left(I-\mathcal{L}^{m-1} I\right)^{-1}\right\| \rightarrow n$ as $t \rightarrow b$. $\int_{t}^{b}\left\|C_{1}(s, t) \tilde{R}(s)\right\| d s \rightarrow 0$ as $t \rightarrow b$ from the condition (3.2.11). Thus, we have

$$
\left\|\left(I-\mathcal{L}^{m-1} I\right)^{-1}\right\| \cdot \int_{t}^{b}\left\|C_{1}(s, t) \tilde{R}(s)\right\| d s \cdot\|\hat{X}\| \| \rightarrow 0 \text { as } t \rightarrow b .
$$

From (3.2.40), (3.2.41) and (3.2.42), we get that $\|\hat{X}-I\| \rightarrow 0$ as $t \rightarrow b$.

Let $P(t)=\hat{X}-I$, we get from (3.2.38) that

$$
P(t)=\sum_{j=1}^{\infty} \mathcal{L}^{j} I
$$

and from (3.2.39),

$$
S(t)=\exp \left(\int_{t_{0}}^{t} D(s) d s\right)(I+\hat{X}(t)-I)=\exp \left(\int_{t_{0}}^{t} D(s) d s\right)(I+P(t))
$$

is a fundamental solution of equation (3.1.1) with $P(t) \rightarrow 0$ as $t \rightarrow b$.

Theorem 3.2.1 with $m=2$ and $b=+\infty$ leads to a simple criterion for asymptotic integration that will be used frequently in the sequel. Therefore, we have the following. 
TheOREM 3.2.4. Let $m=2, b=+\infty$ and assume,

$$
\begin{aligned}
& \text { (i) } \tilde{R}(t) \text { is integrable on }[a, \infty) \text { and } C_{1}(t):=\int_{\infty}^{t} \tilde{R}\left(t_{1}\right) d t_{1} \rightarrow 0 \text { as } t \rightarrow+\infty, \text { and } \\
& \text { (ii) } C_{1}(s) \tilde{R}(s)=\int_{\infty}^{s} \tilde{R}\left(t_{1}\right) d t_{1} \tilde{R}(s) \in L^{1}[a, \infty)
\end{aligned}
$$

then (3.1.1) is right almost diagonal.

Proof. When $m=2$ and $b=\infty$, we have that $\mathcal{L} I=C_{1}(t)$ and $C_{1}(s, t)=\int_{\infty}^{s} \tilde{R}\left(t_{1}\right) d t_{1}=$ $C_{1}(s)$. It is then easy to verify that all conditions of Theorem 3.2.1 hold and the result follows.

Our next result uses integration by parts to render the asymptotic integration of (3.1.1). For the sake of abbreviation we will suppress the explicit mention of the independent variable in $R(s)$ and put $R(s)=R$.

TheOrem 3.2.5. Assume (3.1.1) is potentially oscillatory and the following hold,

$$
\begin{aligned}
& \text { (a) } R \text { is integrable on }[a, \infty) \text { and } \int_{\infty}^{t} R d s \rightarrow 0 \text { as } t \rightarrow \infty, \\
& \text { (b) } D\left(\int_{\infty}^{t} R d s\right)-\left(\int_{\infty}^{t} R d s\right) D \in L^{1}[a, \infty), \\
& \text { (c) }\left(\int_{\infty}^{t} R d s\right) R \in L^{1}[a, \infty), \\
& \text { (d) }\left[\int_{\infty}^{t} \Phi^{-1}\left(D\left(\int_{\infty}^{s} R d \eta\right)-\left(\int_{\infty}^{s} R d \eta\right) D\right) \Phi d s\right] \Phi^{-1} R \Phi \in L^{1}[a, \infty) .
\end{aligned}
$$

Then (3.1.1) is right almost diagonal.

Proof. We need the Proposition 1.1.3 in our proof. Note the following identities. For any differentiable matrix $A(t), B(t)$ and $C(t)$,

$$
\begin{aligned}
A(t) B(t) C(t)-A\left(t_{0}\right) B\left(t_{0}\right) C\left(t_{0}\right) & =\int_{t_{0}}^{t}(A B C)^{\prime} d s \\
& =\int_{t_{0}}^{t} A^{\prime} B C d s+\int_{t_{0}}^{t} A B^{\prime} C d s+\int_{t_{0}}^{t} A B C^{\prime} d s .
\end{aligned}
$$

Therefore,

$$
\int_{t_{0}}^{t} A B^{\prime} C d s=A(t) B(t) C(t)-A\left(t_{0}\right) B\left(t_{0}\right) C\left(t_{0}\right)-\int_{t_{0}}^{t} A^{\prime} B(s) C d s-\int_{t_{0}}^{t} A B C^{\prime} d s .
$$

Let $t_{0}=\infty, A=\Phi^{-1}(t), B=\int_{\infty}^{t} R(s) d s$ and $C=\Phi(t)$. Moreover, by (1.1.9) in Proposition 1.1.3 and condition (3.2.45), we get that $\Phi^{-1} \int_{\infty}^{t} R d s \Phi \rightarrow 0$ as $t \rightarrow \infty$. Hence, 
(3.2.49) implies that

$$
\begin{aligned}
\left|C_{1}(t)\right| & =\left|\int_{\infty}^{t} \tilde{R} d s\right|=\left|\int_{\infty}^{t} \Phi^{-1} R \Phi d s\right| \\
& =\left|\Phi^{-1}\left(\int_{\infty}^{t} R d s\right) \Phi-\int_{\infty}^{t}\left(-\Phi^{-1} D\right)\left(\int_{\infty}^{s} R d \eta\right) \Phi d s-\int_{\infty}^{t} \Phi^{-1}\left(\int_{\infty}^{s} R d \eta\right) D \Phi d s\right| \\
& \leq\left|\Phi^{-1}\left(\int_{\infty}^{t} R d s\right) \Phi\right|+\left|\int_{\infty}^{t} \Phi^{-1}\left(D\left(\int_{\infty}^{s} R d \eta\right)-\left(\int_{\infty}^{s} R d \eta\right) D\right) \Phi d s\right| .
\end{aligned}
$$

Then, (1.1.9), (1.1.10), condition (3.2.45) and (3.2.46) infer that $C_{1}(t) \rightarrow 0$ as $t \rightarrow \infty$.

Next we need the following identity.

$$
\begin{aligned}
C_{1}(s) \tilde{R}(s) & =\left[\Phi^{-1}\left(\int_{\infty}^{s} R d t_{1}\right) \Phi+\int_{\infty}^{s} \Phi^{-1}\left(D\left(\int_{\infty}^{t_{1}} R d \eta\right)-\left(\int_{\infty}^{t_{1}} R d \eta\right) D\right) \Phi d t_{1}\right] \Phi^{-1} R \Phi \\
& =\Phi^{-1}\left(\int_{\infty}^{s} R d t_{1}\right) R \Phi+\left[\int_{\infty}^{s} \Phi^{-1}\left(D\left(\int_{\infty}^{t_{1}} R d \eta\right)-\left(\int_{\infty}^{t_{1}} R d \eta\right) D\right) \Phi d t_{1}\right] \cdot\left(\Phi^{-1} R \Phi\right) .
\end{aligned}
$$

(1.1.10) and condition (3.2.47) and (3.2.48) imply that $C_{1}(s) \tilde{R}(s) \in L^{1}[a, \infty)$.

Therefore, by Theorem 3.2.4, (3.1.1) is right almost diagonal.

REMARK 3.2.6. (a) Evidently, if $R(t) \in L^{1}[a, \infty)$, then with the help of (1.1.10), conditions (3.2.43) and (3.2.44) of Theorem 3.2.4 hold. Thus, we obtain Levinson's theorem for potentially oscillatory systems as a special case of Theorem 3.2.4.

(b) If $\left|\operatorname{Re} \int_{t_{1}}^{t_{2}} \lambda_{j}(s) d s\right| \leq \tilde{M}$, with $\tilde{M}$ a positive fixed constant, then (3.1.1) is a potentially oscillatory system. This includes the special case that the matrix $D(t)+R(t)$ is anti-Hermitian which is of great interest in quantum mechanics.

(c) Although the conditions of Theorem 3.2.4 lead to a theorem that coincides with a result of [12], Theorem 3.2.1 with $m>2$ is new and is not inferred by [12] .

(d) In our Definition 1.1.1 of almost diagonal systems we assume that $r_{k k}(t)=0, k=$ $1,2, \cdots, n$. However, all of our Theorem 3.2.1, Theorem3.2.4 and Theorem 3.2.5 apply to the situation where $r_{k k}(t)$ are not necessarily zero. The assumption that $r_{k k}(t)$ is not necessarily zero is prevalent in the literature on asymptotic integration. See, e.g. [9].

\subsection{Examples}

In this section we consider some examples of potentially oscillatory systems where $R(t)$ possesses elements of the form $\frac{\sin t^{\mu}}{t^{\delta}}$. We know that when $\delta \leq 1, \frac{\sin t^{\mu}}{t^{\delta}}$ does not belong to $L^{1}[a, \infty)$, so that Levison's theorem does not apply. Neither are the examples below included in the analysis of $[\mathbf{9}, \mathbf{1 2}, \mathbf{3 2}]$. However, our Theorem 3.2.4 and Theorem 3.2.5 will show that the differential systems in the following examples are all right almost diagonal.

EXAmple 3.3.1. Consider the system below on the interval $[a, \infty)$ with $a>0$.

$$
Y^{\prime}(t)=\left(\left[\begin{array}{cccc}
\lambda_{1}(t) & 0 & \cdots & 0 \\
0 & \lambda_{2}(t) & \cdots & 0 \\
& \cdots & & \\
0 & 0 & \cdots & \lambda_{n}(t)
\end{array}\right]+\left[\begin{array}{cccc}
0 & c_{12} & \cdots & c_{1 n} \\
c_{21} & 0 & \cdots & c_{2 n} \\
& \cdots & & \\
c_{n 1} & c_{n 2} & \cdots & 0
\end{array}\right] \frac{\sin t^{\mu}}{t^{\delta}}\right) Y(t) .
$$


If the following conditions hold.

(3.3.2) (1) The system is potentially oscillatory,

(2) $\delta, \mu>0,2 \delta+\mu-2>0$ and $\delta+2 \mu-2>0$,

(3) $\frac{\lambda_{k}(s)-\lambda_{j}(s)}{s^{\mu}} \in L^{1}[a, \infty), \frac{\lambda_{k}^{\prime}(s)-\lambda_{j}^{\prime}(s)}{s^{\mu-1}} \in L^{1}[a, \infty)$,

$$
\frac{\left|\lambda_{k}(s)-\lambda_{j}(s)\right|^{2}}{s^{\mu-1}} \in L^{1}[a, \infty), \text { and } \frac{\left|\lambda_{k}(t)-\lambda_{j}(t)\right|}{t^{\mu-1}} \text { is bounded on }[a, \infty) .
$$

Then (3.3.1) is right almost diagonal.

Proof. Notice that the conditions $2 \delta+\mu-2>0$ and $\delta+2 \mu-2>0$ imply

$$
\delta+\mu-1>0 .
$$

In this example,

$$
\tilde{R}(t)=\left(c_{j k} \frac{\sin t^{\mu}}{t^{\delta}} e^{\int_{s_{0}}^{t}\left[\lambda_{k}(s)-\lambda_{j}(s)\right] d s}\right)_{j, k=1}^{n} .
$$

Therefore, in Theorem 3.2.4,

$$
\begin{aligned}
\left(C_{1}(t)\right)_{j k}= & \int_{\infty}^{t} \tilde{r}_{j k}(s) d s \\
= & c_{j k} \int_{\infty}^{t} \frac{\sin s^{\mu}}{s^{\delta}} e^{\int_{s_{0}}^{s}\left[\lambda_{k}(\eta)-\lambda_{j}(\eta)\right] d \eta} d s \\
= & c_{j k}\left[\left(\int_{\infty}^{t} \frac{\sin x^{\mu}}{x^{\delta}} d x\right) e^{\int_{s_{0}}^{t}\left[\lambda_{k}(\eta)-\lambda_{j}(\eta)\right] d \eta}\right. \\
& \left.-\int_{\infty}^{t}\left(\int_{\infty}^{s} \frac{\sin x^{\mu}}{x^{\delta}} d x\right)\left(\lambda_{k}(s)-\lambda_{j}(s)\right) e^{\int_{s_{0}}^{s}\left[\lambda_{k}(\eta)-\lambda_{j}(\eta)\right] d \eta} d s\right] \\
= & c_{j k}\left(A_{1}-A_{2}\right),
\end{aligned}
$$

where

$$
\begin{aligned}
& A_{1}=\left(\int_{\infty}^{t} \frac{\sin x^{\mu}}{x^{\delta}} d x\right) e^{\int_{s_{0}}^{t}\left[\lambda_{k}(\eta)-\lambda_{j}(\eta)\right] d \eta} \\
& A_{2}=\int_{\infty}^{t}\left(\int_{\infty}^{s} \frac{\sin x^{\mu}}{x^{\delta}} d x\right)\left(\lambda_{k}(s)-\lambda_{j}(s)\right) e^{\int_{s_{0}}^{s}\left[\lambda_{k}(\eta)-\lambda_{j}(\eta)\right] d \eta} d s .
\end{aligned}
$$

We now estimate $A_{1}$. Observe that

$$
\begin{aligned}
\int_{\infty}^{t} \frac{\sin x^{\mu}}{x^{\delta}} d x & =\int_{\infty}^{t}\left(-\mu x^{\mu-1} \sin x^{\mu}\right) \frac{1}{-\mu x^{\delta+\mu-1}} d x \\
& =-\frac{\cos t^{\mu}}{\mu t^{\delta+\mu-1}}-\frac{\delta+\mu-1}{\mu} \int_{\infty}^{t}\left(\cos x^{\mu}\right) \frac{1}{x^{\delta+\mu}} d x
\end{aligned}
$$

which leads to

$$
\left|\int_{\infty}^{t} \frac{\sin x^{\mu}}{x^{\delta}} d x\right| \leq \frac{1}{\mu t^{\delta+\mu-1}}+\frac{\delta+\mu-1}{\mu} \int_{t}^{\infty} \frac{1}{x^{\delta+\mu}} d x=\frac{2}{\mu t^{\delta+\mu-1}}
$$


Since the system is potentially oscillatory, there exists $M_{1}>0$ such that

$$
\left|e^{\int_{s_{0}}^{t}\left[\lambda_{k}(\eta)-\lambda_{j}(\eta)\right] d \eta}\right| \leq \frac{\mu M_{1}}{2}
$$

for $t \in[a, \infty)$. Therefore,

$$
\left|A_{1}\right|=\left|\int_{\infty}^{t} \frac{\sin x^{\mu}}{x^{\delta}} d x\right| \cdot\left|e^{\int_{s_{0}}^{t}\left[\lambda_{k}(\eta)-\lambda_{j}(\eta)\right] d \eta}\right| \leq \frac{2}{\mu t^{\delta+\mu-1}} \cdot \frac{\mu M_{1}}{2}=\frac{M_{1}}{t^{\delta+\mu-1}} .
$$

Then we consider $A_{2}$. First observe that by integration by parts we have

$$
\begin{aligned}
\int_{\infty}^{t}\left(\cos x^{\mu}\right) \frac{1}{x^{\delta+\mu}} d x & =\int_{\infty}^{t} \mu x^{\mu-1} \cos x^{\mu} \frac{1}{\mu x^{\delta+2 \mu-1}} d x \\
& =\frac{\sin t^{\mu}}{\mu t^{\delta+2 \mu-1}}+\frac{\delta+2 \mu-1}{\mu} \int_{\infty}^{t} \frac{\sin x^{\mu}}{x^{\delta+2 \mu}} d x .
\end{aligned}
$$

Therefore, insert (3.3.14) into (3.3.10) to get

$$
\begin{aligned}
\int_{\infty}^{t} \frac{\sin x^{\mu}}{x^{\delta}} d x= & -\frac{\cos t^{\mu}}{\mu t^{\delta+\mu-1}}-\frac{\delta+\mu-1}{\mu^{2}} \frac{\sin t^{\mu}}{t^{\delta+2 \mu-1}} \\
& -\frac{(\delta+\mu-1)(\delta+2 \mu-1)}{\mu^{2}} \int_{\infty}^{t} \frac{\sin x^{\mu}}{x^{\delta+2 \mu}} d x .
\end{aligned}
$$

Insert (3.3.15) into (3.3.9) and we get

$$
\begin{aligned}
\left|A_{2}\right| \leq\left|\frac{1}{\mu} \int_{\infty}^{t} \frac{\cos s^{\mu}}{s^{\delta+\mu-1}}\left(\lambda_{k}(s)-\lambda_{j}(s)\right) e^{\int_{s_{0}}^{s}\left[\lambda_{k}(\eta)-\lambda_{j}(\eta)\right] d \eta} d s\right| \\
+\left|\int_{\infty}^{t}\left[\frac{\delta+\mu-1}{\mu^{2}} \cdot \frac{\sin s^{\mu}}{s^{\delta+2 \mu-1}}\right]\left(\lambda_{k}(s)-\lambda_{j}(s)\right) e^{\int_{s_{0}}^{s}\left[\lambda_{k}(\eta)-\lambda_{j}(\eta)\right] d \eta} d s\right| \\
(3.3 .16)+\left|\int_{\infty}^{t}\left[\frac{(\delta+\mu-1)(\delta+2 \mu-1)}{\mu^{2}} \int_{\infty}^{s} \frac{\sin x^{\mu}}{x^{\delta+2 \mu}} d x\right]\left(\lambda_{k}(s)-\lambda_{j}(s)\right) e^{\int_{s_{0}}^{s}\left[\lambda_{k}(\eta)-\lambda_{j}(\eta)\right] d \eta} d s\right| .
\end{aligned}
$$

Since $\frac{\lambda_{k}(s)-\lambda_{j}(s)}{s^{\mu}} \in L^{1}$, there exists some $M_{2}>0$ such that $\int_{t}^{\infty}\left|\frac{\lambda_{k}(s)-\lambda_{j}(s)}{s^{\mu}}\right| d s \leq M_{2}$ when $t \in[a, \infty)$. Therefore,

$$
\begin{aligned}
& \left|\int_{\infty}^{t}\left[\frac{\delta+\mu-1}{\mu^{2}} \cdot \frac{\sin s^{\mu}}{s^{\delta+2 \mu-1}}\right]\left(\lambda_{k}(s)-\lambda_{j}(s)\right) e^{\int_{s_{0}}^{s}\left[\lambda_{k}(\eta)-\lambda_{j}(\eta)\right] d \eta} d s\right| \\
\leq & \frac{\delta+\mu-1}{\mu^{2}} \int_{t}^{\infty} \frac{1}{s^{\delta+\mu-1}} \cdot \frac{\left|\lambda_{k}(s)-\lambda_{j}(s)\right|}{s^{\mu}} \cdot\left|e^{\int_{s_{0}}^{s}\left[\lambda_{k}(\eta)-\lambda_{j}(\eta)\right] d \eta}\right| d s \\
\leq & \frac{\delta+\mu-1}{\mu^{2}} \cdot \frac{1}{t^{\delta+\mu-1}} \int_{t}^{\infty} \frac{\left|\lambda_{k}(s)-\lambda_{j}(s)\right|}{s^{\mu}} d s \cdot \frac{\mu M_{1}}{2} \\
\leq & \frac{M_{3}}{t^{\delta+\mu-1}}
\end{aligned}
$$

where $M_{3}=\frac{1}{\mu}(\delta+\mu-1) \cdot M_{2} \cdot \frac{M_{1}}{2}$. 
Similar to the estimation in (3.3.17) we have

$$
\begin{aligned}
&\left|\int_{\infty}^{t}\left[\frac{(\delta+\mu-1)(\delta+2 \mu-1)}{\mu^{2}} \int_{\infty}^{s} \frac{\sin x^{\mu}}{x^{\delta+2 \mu}} d x\right]\left(\lambda_{k}(s)-\lambda_{j}(s)\right) e^{\int_{s_{0}}^{s}\left[\lambda_{k}(\eta)-\lambda_{j}(\eta)\right] d \eta} d s\right| \\
& \leq \frac{\delta+\mu-1}{\mu^{2}} \int_{t}^{\infty}\left((\delta+2 \mu-1) \int_{s}^{\infty} \frac{1}{x^{\delta+2 \mu}} d x\right) \cdot\left|\lambda_{k}(s)-\lambda_{j}(s)\right| \cdot\left|e^{\int_{s_{0}}^{s}\left[\lambda_{k}(\eta)-\lambda_{j}(\eta)\right] d \eta}\right| d s \\
&= \frac{\delta+\mu-1}{\mu^{2}} \int_{t}^{\infty} \frac{1}{s^{\delta+2 \mu-1}} \cdot\left|\lambda_{k}(s)-\lambda_{j}(s)\right| \cdot\left|e^{\int_{s_{0}}^{s}\left[\lambda_{k}(\eta)-\lambda_{j}(\eta)\right] d \eta}\right| d s \\
& \text { (3.3.18) } \leq \frac{M_{3}}{t^{\delta+\mu-1}} .
\end{aligned}
$$

Using integration by parts yields

$$
\begin{aligned}
& \int_{\infty}^{t} \frac{\cos s^{\mu}}{s^{\delta+\mu-1}}\left(\lambda_{k}(s)-\lambda_{j}(s)\right) e^{\int_{s_{0}}^{s}\left[\lambda_{k}(\eta)-\lambda_{j}(\eta)\right] d \eta} d s \\
= & \left(\int_{\infty}^{t} \frac{\cos x^{\mu}}{x^{\delta+\mu-1}} d x\right)\left(\lambda_{k}(t)-\lambda_{j}(t)\right) e^{\int_{s_{0}}^{t}\left[\lambda_{k}(\eta)-\lambda_{j}(\eta)\right] d \eta} \\
& -\int_{\infty}^{t}\left(\int_{\infty}^{s} \frac{\cos x^{\mu}}{x^{\delta+\mu-1}} d x\right)\left[\left(\lambda_{k}(s)-\lambda_{j}(s)\right)^{\prime}+\left(\lambda_{k}(s)-\lambda_{j}(s)\right)^{2}\right] e^{\int_{s_{0}}^{s}\left[\lambda_{k}(\eta)-\lambda_{j}(\eta)\right] d \eta} d s,
\end{aligned}
$$

and

$$
\begin{aligned}
\left|\int_{\infty}^{t} \frac{\cos x^{\mu}}{x^{\delta+\mu-1}} d x\right| & =\left|\frac{\sin t^{\mu}}{\mu t^{\delta+2 \mu-2}}+\frac{\delta+2 \mu-2}{\mu} \int_{\infty}^{t} \frac{\sin x^{\mu}}{x^{\delta+2 \mu-1}} d x\right| \\
& \leq \frac{1}{\mu t^{\delta+2 \mu-2}}+\frac{\delta+2 \mu-2}{\mu} \int_{t}^{\infty} \frac{1}{x^{\delta+2 \mu-1}} d x \\
& =\frac{2}{\mu t^{\delta+2 \mu-2}} .
\end{aligned}
$$

Combine now the estimations in (3.3.19) and (3.3.20) to get

$$
\begin{aligned}
& \left|\int_{\infty}^{t} \frac{\cos s^{\mu}}{s^{\delta+\mu-1}}\left(\lambda_{k}(s)-\lambda_{j}(s)\right) e^{\int_{s_{0}}^{s}\left[\lambda_{k}(\eta)-\lambda_{j}(\eta)\right] d \eta} d s\right| \\
\leq & \frac{2}{\mu t^{\delta+2 \mu-2}} \cdot\left|\lambda_{k}(t)-\lambda_{j}(t)\right| \cdot \mid e^{\int_{s_{0}}^{t}\left[\lambda_{k}(\eta)-\lambda_{j}(\eta)\right] d \eta \mid} \\
& \quad+\int_{t}^{\infty} \frac{2}{\mu s^{\delta+2 \mu-2}}\left(\left|\lambda_{k}^{\prime}(s)-\lambda_{j}^{\prime}(s)\right|+\left|\lambda_{k}(s)-\lambda_{j}(s)\right|^{2}\right)\left|e^{\int_{s_{0}}^{s}\left[\lambda_{k}(\eta)-\lambda_{j}(\eta)\right] d \eta}\right| d s \\
\leq & \frac{\left|\lambda_{k}(t)-\lambda_{j}(t)\right|}{t^{\mu-1}} \cdot \frac{M_{1}}{t^{\delta+\mu-1}}+\frac{1}{t^{\delta+\mu-1}} \int_{t}^{\infty} M_{1} \frac{\left|\lambda_{k}^{\prime}(s)-\lambda_{j}^{\prime}(s)\right|+\left|\lambda_{k}(s)-\lambda_{j}(s)\right|^{2}}{s^{\mu-1}} d s \\
(3.3 .21) \leq & \frac{\mu M_{4}}{t^{\delta+\mu-1}},
\end{aligned}
$$

where $M_{4}$ is a positive constant. We applied the conditions that $\frac{\left|\lambda_{k}(t)-\lambda_{j}(t)\right|}{t^{\mu-1}}$ is bounded on $[a,+\infty), \frac{\left|\lambda_{k}^{\prime}(s)-\lambda_{j}^{\prime}(s)\right|}{s^{\mu-1}} \in L^{1}$ and $\frac{\left|\lambda_{k}(s)-\lambda_{j}(s)\right|^{2}}{s^{\mu-1}} \in L^{1}$ in $(3.3 .21)$.

$>$ From (3.3.16), (3.3.17), (3.3.18) and (3.3.21), we get

$$
\left|A_{2}\right| \leq \frac{2 M_{3}+M_{4}}{t^{\delta+\mu-1}} .
$$


The identity (3.3.7) and the estimates on $A_{1}$ and $A_{2}$ in (3.3.13) and (3.3.22) help us conclude that,

$$
\left|\left(C_{1}(t)\right)_{j k}\right| \leq\left|c_{j k}\right| \cdot\left(\left|A_{1}\right|+\left|A_{2}\right|\right) \leq\left|c_{j k}\right|\left(\frac{M_{1}}{t^{\delta+\mu-1}}+\frac{2 M_{3}+M_{4}}{t^{\delta+\mu-1}}\right) \leq \frac{M}{t^{\delta+\mu-1}},
$$

where $M=\max \left\{\left|c_{j k}\right|\right\}\left(M_{1}+2 M_{3}+M_{4}\right)$ is a positive number.

By (3.3.5) and (3.3.23), we get that condition (3.2.43) of Theorem 3.2.4 holds, namely $\tilde{R}$ is integrable on $[a, \infty)$ and $C_{1}(t) \rightarrow 0$ as $t \rightarrow \infty$.

Let us show that condition (3.2.44) of Theorem 3.2.4 also holds. We have

$$
\begin{aligned}
\left|\left(C_{1}(s) \tilde{R}(s)\right)_{j k}\right| & =\left|\sum_{l=1}^{n}\left(\int_{\infty}^{s} \tilde{r}_{j l}\left(t_{1}\right) d t_{1} \cdot \tilde{r}_{l k}(s)\right)\right| \leq \sum_{l=1}^{n}\left|\int_{\infty}^{s} \tilde{r}_{j l}\left(t_{1}\right) d t_{1}\right| \cdot\left|\tilde{r}_{l k}(s)\right| \\
& \leq \sum_{l=1}^{n} \frac{M}{s^{\delta+\mu-1}} \cdot c \frac{\mu M_{1}}{2}\left|\frac{\sin s^{\mu}}{s^{\delta}}\right| \leq \frac{c n \mu M M_{1}}{2 s^{2 \delta+\mu-1}}
\end{aligned}
$$

where $c=\operatorname{Max}\left\{\left|c_{j k}\right|\right\}$. Since $2 \delta+\mu-1>1$, we have that $C_{1}(s) \tilde{R}(s) \in L^{1}$.

Therefore, the system (3.3.1) is right almost diagonal.

$>$ From Example 3.3.1, we can see that the differential system

$$
Y^{\prime}(t)=\left(\left[\begin{array}{cc}
t i+\frac{i}{t} & 0 \\
0 & t i-\frac{i}{t}
\end{array}\right]+\left[\begin{array}{cc}
0 & c_{1} \\
c_{2} & 0
\end{array}\right] \frac{\sin t^{2}}{t^{\frac{1}{4}}}\right) Y(t)
$$

with $i=\sqrt{-1}$, is right almost diagonal. However, Levison's Theorem and other theorems in [9] do not apply. Neither does Theorem 2.1 in [32] because $D(t) \int_{\infty}^{t} R(s) d s$ does not belong to $L^{1}$.

At the same time, from Example 3.3.1 we can get that

$$
Y^{\prime}(t)=\left(\left[\begin{array}{cc}
1 & 0 \\
0 & -1
\end{array}\right] \frac{1}{t^{2}}+\left[\begin{array}{cc}
0 & c_{1} \\
c_{2} & 0
\end{array}\right] \frac{\sin t}{t^{\frac{3}{4}}}\right) Y(t)
$$

is right almost diagonal. Theorem 1 in [12] (Theorem 1.1.12 of our work) does not apply because

$$
\frac{r_{21}(t)}{\lambda_{1}(t)-\lambda_{2}(t)}=\frac{c_{2} \frac{\sin t}{t^{\frac{3}{4}}}}{\frac{2}{t^{2}}}=\frac{c_{2}}{2} \sin t \cdot t^{\frac{5}{4}}
$$

which does not goes to 0 as $t \rightarrow \infty$.

We can obtain stronger results if we narrow our discussion to the case $n=2$.

EXAMPlE 3.3.2. Consider the differential system

$$
Y^{\prime}(t)=\left(\left[\begin{array}{cc}
\lambda_{1}(t) & 0 \\
0 & \lambda_{2}(t)
\end{array}\right]+\left[\begin{array}{cc}
0 & r_{12}(t) \\
r_{21}(t) & 0
\end{array}\right]\right) Y(t)
$$


where $r_{12}(t)=c_{12} \frac{\sin t^{\mu_{1}}}{t^{\delta_{1}}}, r_{21}(t)=c_{21} \frac{\sin t^{\mu_{2}}}{t^{\delta_{2}}}$. Let,

(3.3.25) (1) the system is potentially oscillatory on $[a, \infty)$ with $a>0$;

(2) for $j=1,2, \delta_{j}, \mu_{j}>0, \delta_{j}+\mu_{j}-1>0, \delta_{j}+2 \mu_{j}-2>0$ and $\delta_{1}+\delta_{2}+\mu_{j}-2>0$

(3) for $j=1,2, \frac{\lambda_{1}(s)-\lambda_{2}(s)}{s^{\mu_{j}}} \in L^{1}[a, \infty), \frac{\lambda_{1}^{\prime}(s)-\lambda_{2}^{\prime}(s)}{s^{\mu_{j}-1}} \in L^{1}[a, \infty)$,

$$
\frac{\left|\lambda_{1}(s)-\lambda_{2}(s)\right|^{2}}{s^{\mu_{j}-1}} \in L^{1}[a, \infty) \text {, and } \frac{\left|\lambda_{1}(t)-\lambda_{2}(t)\right|}{t^{\mu_{j}-1}} \text { is bounded on }[a,+\infty),
$$

Then (3.3.24) is right almost diagonal.

ProOF. In this example,

$$
\tilde{r}_{12}(t)=c_{12} \frac{\sin t^{\mu_{1}}}{t^{\delta_{1}}} e^{\int_{t_{0}}^{t}\left[\lambda_{2}(s)-\lambda_{1}(s)\right] d s} \quad, \quad \tilde{r}_{21}(t)=c_{21} \frac{\sin t^{\mu_{2}}}{t^{\delta_{2}}} e^{\int_{t_{0}}^{t}\left[\lambda_{1}(s)-\lambda_{2}(s)\right] d s} .
$$

Similar to the analysis of Example 3.3.1, conditions (3.3.25), the first three inequalities of (3.3.26) and conditions of (3.3.27) leads to that $C_{1}(t) \rightarrow 0$ as $t \rightarrow+\infty$.

At the same time, notice that,

$$
\begin{aligned}
\left|C_{1}(s) \tilde{R}(s)\right| & =\left[\begin{array}{cc}
\left|\tilde{r}_{21}(s) \int_{\infty}^{s} \tilde{r}_{12}\left(t_{1}\right) d t_{1}\right| & 0 \\
0 & \left|\tilde{r}_{12}(s) \int_{\infty}^{s} \tilde{r}_{21}\left(t_{1}\right) d t_{1}\right|
\end{array}\right] \\
& \leq\left[\begin{array}{cc}
K\left|c_{21} \frac{\sin s^{\mu_{2}}}{s^{\delta_{2}}}\right| \cdot\left|\frac{M}{s^{\delta_{1}+\mu_{1}-1}}\right| & \\
0 & K\left|c_{12} \frac{\sin s^{\mu_{1}}}{s^{\delta_{1}}}\right| \cdot\left|\frac{M}{s^{\delta_{2}+\mu_{2}-1}}\right|
\end{array}\right] \\
& \leq K\left[\begin{array}{cc}
\frac{\left|c c_{21}\right| M}{s^{\delta_{1}+\delta 2+\mu_{1}-1}} & 0 \\
0 & \frac{\left|c_{12}\right| M}{s^{\delta_{1}+\delta 2+\mu_{2}-1}}
\end{array}\right]
\end{aligned}
$$

where $K>0$ is a constant such that $\left|e^{\int_{t_{0}}^{t}\left[\lambda_{1}(s)-\lambda_{2}(s)\right]} d s\right|<K$ when $t \in[a, \infty)$.

With the help of $\delta_{1}+\delta_{2}+\mu_{j}-1>1, j=1,2$, we get that $C_{1}(s) \tilde{R}(s) \in L^{1}$.

Now we want to apply Theorem 3.2.5 to the differential system in Example 3.3.2.

ExAmple 3.3.3. Consider the same differential system (3.3.24) in Example 3.3.2, if

(1) the system is potentially oscillatory on $[a, \infty)$ with $a>0$,

$$
\text { and }\left|\lambda_{1}(t)-\lambda_{2}(t)\right| \leq \frac{C}{t}, \text { where } C>0 \text { is a constant; }
$$

(2) $\delta_{j}, \mu_{j}>0, \delta_{j}+\mu_{j}-1>0$, and $\delta_{1}+\delta_{2}+\mu_{j}-2>0, j=1,2$,

then (3.3.24) is right almost diagonal.

Proof. First, from (3.3.11) in the proof of Example 3.3.1, we know that

$$
\left|\int_{\infty}^{t} \frac{\sin x^{\mu_{j}}}{x^{\delta_{j}}} d x\right| \leq \frac{2}{\mu_{j} t^{\delta_{j}+\mu_{j}-1}}, j=1,2
$$

Therefore, $\int_{\infty}^{t} R d s \rightarrow 0$ as $t \rightarrow \infty$.

Second, we have

$$
D\left(\int_{\infty}^{t} R d s\right)-\left(\int_{\infty}^{t} R d s\right) D=\left[\begin{array}{cc}
0 & \left(\lambda_{1}-\lambda_{2}\right) \int_{\infty}^{t} r_{12} d s \\
\left(\lambda_{2}-\lambda_{1}\right) \int_{\infty}^{t} r_{21} d s & 0
\end{array}\right] .
$$


Also we know that

$$
\begin{aligned}
\left|\left(\lambda_{1}-\lambda_{2}\right) \int_{\infty}^{t} r_{12} d s\right| & =\left|\lambda_{1}-\lambda_{2}\right| \cdot\left|\int_{\infty}^{t} r_{12} d s\right| \\
& \leq \frac{C}{t} \cdot \frac{2\left|c_{12}\right|}{\mu_{1} t^{\delta_{1}+\mu_{1}-1}} \\
& =\frac{2 C\left|c_{12}\right|}{\mu_{1} t^{\delta_{1}+\mu_{1}}}
\end{aligned}
$$

and similarly,

$$
\left|\left(\lambda_{2}-\lambda_{1}\right) \int_{\infty}^{t} r_{21} d s\right| \leq \frac{2 C\left|c_{21}\right|}{\mu_{2} t^{\delta_{2}+\mu_{2}}} .
$$

Since $\delta_{j}+\mu_{j}-1>0, j=1,2$, we conclude that $D\left(\int_{\infty}^{t} R d s\right)-\left(\int_{\infty}^{t} R d s\right) D \in L^{1}[a, \infty)$.

Third,

$$
\begin{aligned}
\left|\left(\int_{\infty}^{t} R d s\right) R\right| & =\left[\begin{array}{cc}
0 & \left|\int_{\infty}^{t} r_{12} d s\right| \\
\left|\int_{\infty}^{t} r_{21} d s\right| & 0
\end{array}\right]\left[\begin{array}{cc}
0 & \left|r_{12}\right| \\
\left|r_{21}\right| & 0
\end{array}\right] \\
& =\left[\begin{array}{cc}
\left|\int_{\infty}^{t} r_{12} d s\right| \cdot\left|r_{21}\right| & 0 \\
0 & \left|\int_{\infty}^{t} r_{21} d s\right| \cdot\left|r_{12}\right|
\end{array}\right] \\
& \leq\left|c_{12} c_{21}\right|\left[\begin{array}{cc}
\frac{2}{\mu_{1} t^{\delta_{1}+\mu_{1}-1}} \cdot \frac{1}{t^{\delta_{2}}} & 0 \\
0 & \frac{2}{\mu_{2} t^{\delta_{2}+\mu_{2}-1}} \cdot \frac{1}{t^{\delta_{1}}}
\end{array}\right] \\
& =\left|c_{12} c_{21}\right|\left[\begin{array}{cc}
\frac{2}{\mu_{1} t^{\delta_{1}+\delta_{2}+\mu_{1}-1}} & 0 \\
0 & \frac{2}{\mu_{2} t^{\delta_{1}+\delta_{2}+\mu_{2}-1}}
\end{array}\right] .
\end{aligned}
$$

Since $\delta_{1}+\delta_{2}+\mu_{j}-2>0, j=1,2$, we have $\left(\int_{\infty}^{t} R d s\right) R \in L^{1}[a, \infty)$.

At last, as the system is potentially oscillatory, with the help of (1.1.9) and (1.1.10), we get

$$
\begin{aligned}
& \left|\left[\int_{\infty}^{t} \Phi^{-1}\left(D\left(\int_{\infty}^{s} R d \eta\right)-\left(\int_{\infty}^{s} R d \eta\right) D\right) \Phi d s\right] \Phi^{-1} R \Phi\right| \\
& \leq M_{5}\left[\begin{array}{cc}
0 & 0 \\
\int_{t}^{\infty}\left|\left(\lambda_{2}-\lambda_{1}\right) \int_{\infty}^{s} r_{21} d t_{1}\right| d s & \int_{t}^{\infty}\left|\left(\lambda_{1}-\lambda_{2}\right) \int_{\infty}^{s} r_{12} d t_{1}\right| d s
\end{array}\right]\left[\begin{array}{cc}
0 & \left|r_{12}\right| \\
\left|r_{21}\right| & 0
\end{array}\right] \\
& \leq M_{6}\left[\begin{array}{cc}
0 & \int_{t}^{\infty} \frac{2 C}{s^{\delta_{1}+\mu_{1}}} d s \\
\int_{t}^{\infty} \frac{2 C}{s^{\delta_{2}+\mu_{2}}} d s & 0
\end{array}\right]\left[\begin{array}{cc}
0 & \frac{1}{t^{\delta_{1}}} \\
\frac{1}{t^{\delta_{2}}} & 0
\end{array}\right] \\
& \leq M_{7}\left[\begin{array}{cc}
\frac{1}{t^{\delta_{1}+\delta_{2}+\mu_{1}-1}} & 0 \\
0 & \frac{1}{t^{\delta_{1}+\delta_{2}+\mu_{2}-1}}
\end{array}\right]
\end{aligned}
$$

where $M_{5}, M_{6}$ and $M_{7}$ are some positive numbers.

Therefore, $\left[\int_{\infty}^{t} \Phi^{-1}\left(D\left(\int_{\infty}^{s} R d \eta\right)-\left(\int_{\infty}^{s} R d \eta\right) D\right) \Phi d s\right] \Phi^{-1} R \Phi \in L^{1}[a, \infty)$.

By Theorem 3.2.5, we know that (3.3.24) is right almost diagonal. 
CHAPTER 4

\section{Right almost diagonal difference equations}

\subsection{Introduction}

We will study the matrix difference equation

$$
Y(t+1)=(D(t)+R(t)) Y(t), \quad t \geq a
$$

in which

$$
\begin{aligned}
D(t) & =\operatorname{diag}\left\{\lambda_{1}(t), \lambda_{2}(t), \ldots, \lambda_{n}(t)\right\}, \\
R(t) & =\left\{r_{j k}(t)\right\}_{j, k=1}^{n}, \text { with } r_{k k}(t)=0 .
\end{aligned}
$$

This chapter is devoted to set up a new set of conditions on linear difference equations, especially "potentially oscillatory". difference equations, under which (4.1.1) is a right almost diagonal system, i.e. it possesses an asymptotic representation

$$
Y(t)=\left(\prod_{l=a}^{t-1} D(l)\right)(I+P(t))
$$

with $P(t) \rightarrow 0$ as $t \rightarrow \infty$.

The meaning of potentially oscillatory for a difference system $(4.1 .1)$ on $[a, \infty)$ is

$$
0<M_{2} \leq\left|\prod_{l=t_{1}}^{t_{2}} \frac{\lambda_{k}(l)}{\lambda_{j}(l)}\right| \leq M_{1}, \quad j, k=1,2, \cdots n, \quad j \neq k,
$$

for all $a \leq t_{1}<t_{2} \leq \infty$ and $M_{1}$ and $M_{2}$ are fixed positive numbers.

In Section 4.2, we employ a technique to convert a difference system for $P(t)$ into a family of summable equations. Each member of this family points to a different theorem of asymptotic summation. Conditions will be given which will guarantee that the system (4.1.1) is right almost diagonal. Examples and comparisons will be discussed in Section 4.3.

\subsection{Asymptotic Summation}

It can be verified that a fundamental solution $Y(t)$ of (4.1.1) can be written as

$$
Y(t)=\Phi(t) Z(t)
$$

where $\Phi(t)$ is a fundamental matrix solution of

$$
\Phi(t+1)=D(t) \Phi(t)
$$


and $Z(t)$ is a fundamental matrix solution of

$$
\triangle Z(t)=\tilde{R}(t) Z(t)
$$

with

$$
\tilde{R}(t)=\Phi^{-1}(t+1) R(t) \Phi(t)
$$

Indeed, by (4.2.3), we have $Z(t+1)=(I+\tilde{R}(t)) Z(t)$. Therefore, if $Y(t)=\Phi(t) Z(t)$, we can obtain

$$
\begin{aligned}
Y(t+1) & =\Phi(t+1) Z(t+1) \\
& =\Phi(t+1)\left(I+\Phi^{-1}(t+1) R(t) \Phi(t)\right) Z(t) \\
& =\Phi(t+1) Z(t)+R(t) \Phi(t) Z(t) \\
& =D(t) \Phi(t) Z(t)+R(t) \Phi(t) Z(t) \\
& =(D(t)+R(t)) Y(t) .
\end{aligned}
$$

When $a \leq t<b$, with $a<b \leq \infty$, assume $\lambda_{j}(t) \neq 0$ for $j=1,2, \cdots, n$. Then, it is easy to find an invertible matrix solution of (4.2.2) that is

$$
\Phi(t)=\prod_{l=a}^{t-1} D(l)=\operatorname{Diag}\left\{\prod_{l=a}^{t-1} \lambda_{1}(l), \prod_{l=a}^{t-1} \lambda_{2}(l), \cdots, \prod_{l=a}^{t-1} \lambda_{n}(l)\right\} .
$$

In order to find a fundamental matrix solution of (4.2.3), for any $n \times n$ matrix $Z(t)$, we define a linear operator $\mathcal{L}$ by

$$
(\mathcal{L} Z)(t):=-\sum_{t_{1}=t}^{b} \tilde{R}\left(t_{1}\right) Z\left(t_{1}\right)
$$

provided that the summation exists for $a \leq t<b$. It is then evident that $Z(t)$ is a fundamental solution of (4.2.3) if $Z(t)$ satisfies the equation

$$
Z(t)=I+\mathcal{L} Z(t)
$$

Define for an integer $m$ with $m \geq 2$, the matrix function $C\left(t_{1}, t\right)$ by

$$
C\left(t_{1}, t\right)=\sum_{t_{2}=t_{1}+1}^{b} \sum_{t_{3}=t}^{t_{2}} \sum_{t_{4}=t}^{t_{3}} \cdots \sum_{t_{m}=t}^{t_{m-1}} \tilde{R}\left(t_{m}\right) \tilde{R}\left(t_{m-1}\right) \cdots \tilde{R}\left(t_{2}\right) .
$$

An important result of this chapter is the following.

THEOREM 4.2.1. Assume that for some integer $m \geq 2$ we have uniformly for $b \leq \infty$ that in (4.2.6) and (4.2.8)

(i) $\mathcal{L}^{k} I, k=1,2, \cdots, m-1$, are convergent summations for $t \in[a, b)$,

$$
\text { (ii) } \quad \mathcal{L}^{k} I \rightarrow 0 \text { as } t \rightarrow b, \text { for } k=1,2, \cdots, m-1 \text {, and }
$$

$$
\text { (iii) } \sum_{t_{1}=t}^{b}\left\|C\left(t_{1}, t\right) \tilde{R}\left(t_{1}\right)\right\| \rightarrow 0 \text { as } t \rightarrow b \text {. }
$$


Then, there exists an integer a large enough such that for $t \geq a$, the resolvent series $\sum_{j=1}^{\infty} \mathcal{L}^{j} I$ is absolutely and uniformly convergent. Moreover, the difference system (4.1.1) is right almost diagonal. Namely, a fundamental solution $Y(t)$ is given by

$$
Y(t)=\left(\prod_{l=a}^{t-1} D(l)\right)(I+P(t))
$$

with $P(t)=\sum_{j=1}^{\infty} \mathcal{L}^{j} I \rightarrow 0$ as $t \rightarrow b$.

We need first some preparatory lemmas. The first lemma below is about changing the order of summation in certain expression.

Lemma 4.2.2. Suppose $f: \mathbb{R}^{m} \rightarrow \mathbb{R}$ with $m \geq 2$, is a summable function, when $t \leq b \leq$ $\infty$, we have

$$
\begin{aligned}
& \sum_{t_{m}=t}^{b} \sum_{t_{m}=t_{m}}^{b} \sum_{t_{m-2}=t_{m-1}}^{b} \cdots \sum_{t_{1}=t_{2}}^{b} f\left(t_{1}, t_{2}, \cdots, t_{m}\right) \\
= & \sum_{t_{1}=t}^{b} \sum_{t_{2}=t}^{t_{1}} \sum_{t_{3}=t}^{t_{2}} \cdots \sum_{t_{m}=t}^{t_{m-1}} f\left(t_{1}, t_{2}, \cdots, t_{m}\right) .
\end{aligned}
$$

ProOF. By induction, when $m=2$, we observe that

$$
\sum_{t_{2}=t}^{b} \sum_{t_{1}=t_{2}}^{b} f\left(t_{1}, t_{2}\right)=\sum_{t_{1}=t}^{b} \sum_{t_{2}=t}^{t_{1}} f\left(t_{1}, t_{2}\right)
$$

Assume that (4.2.12) is valid for $2 \leq m \leq k-1$. When $m=k$,

$$
\sum_{t_{k}=t}^{b} \sum_{t_{k-1}=t_{k}}^{b} \sum_{t_{k-2}=t_{k-1}}^{b} \cdots \sum_{t_{1}=t_{2}}^{b} f\left(t_{1}, t_{2}, \cdots, t_{k}\right)=\sum_{t_{k}=t}^{b} \sum_{t_{k-1}=t_{k}}^{b} g\left(t_{k-1}, t_{k}\right),
$$

where $g\left(t_{k-1}, t_{k}\right)=\sum_{t_{k-2}=t_{k-1}}^{b} \cdots \sum_{t_{1}=t_{2}}^{b} f\left(t_{1}, t_{2}, \cdots, t_{k}\right)$. Changing the order of summation in $(4.2 .13)$, we get

$$
\begin{aligned}
\sum_{t_{k}=t}^{b} \sum_{t_{k-1}=t_{k}}^{b} g\left(t_{k-1}, t_{k}\right) & =\sum_{t_{k-1}=t}^{b} \sum_{t_{k}=t}^{t_{k-1}} g\left(t_{k-1}, t_{k}\right) \\
& =\sum_{t_{k-1}=t}^{b} \sum_{t_{k}=t}^{t_{k-1}} \sum_{t_{k-2}=t_{k-1}}^{b} \cdots \sum_{t_{1}=t_{2}}^{b} f\left(t_{1}, t_{2}, \cdots, t_{k}\right) \\
& =\sum_{t_{k-1}=t}^{b} \sum_{t_{k-2}=t_{k-1}}^{b} \cdots \sum_{t_{1}=t_{2}}^{b}\left(\sum_{t_{k}=t}^{t_{k-1}} f\left(t_{1}, t_{2}, \cdots, t_{k}\right)\right) \\
& =\sum_{t_{k-1}=t}^{b} \sum_{t_{k-2}=t_{k-1}}^{b} \cdots \sum_{t_{1}=t_{2}}^{b} h\left(t_{1}, t_{2}, \cdots, t_{k-1}\right),
\end{aligned}
$$

in which $h\left(t_{1}, t_{2}, \cdots, t_{k-1}\right)=\sum_{t_{k}=t}^{t_{k-1}} f\left(t_{1}, t_{2}, \cdots, t_{k}\right)$. 
By the assumption, we change the order of summation in the last term of (4.2.14) to get

$$
\begin{aligned}
\sum_{t_{k-1}=t}^{b} \sum_{t_{k-2}=t_{k-1}}^{b} \cdots \sum_{t_{1}=t_{2}}^{b} h\left(t_{1}, t_{2}, \cdots, t_{k-1}\right) & =\sum_{t_{1}=t}^{b} \sum_{t_{2}=t}^{t_{1}} \cdots \sum_{t_{k-1}=t}^{t_{k-2}} h\left(t_{1}, t_{2}, \cdots, t_{k-1}\right) \\
& =\sum_{t_{1}=t}^{b} \sum_{t_{2}=t}^{t_{1}} \cdots \sum_{t_{k-1}=t}^{t_{k-2}} \sum_{t_{k}=t}^{t_{k-1}} f\left(t_{1}, t_{2}, \cdots, t_{k}\right) .
\end{aligned}
$$

Therefore, we conclude that (4.2.12) is valid for any $m \in \mathbb{N}$ with $m \geq 2$.

We now need to embed the difference system (4.1.1) in a wider family of difference equations. Consider the difference system

$$
\hat{Y}(t+1, \epsilon)=[D(t)+\epsilon R(t)] \hat{Y}(t, \epsilon),
$$

in which $\epsilon \in D_{\rho}$ with $D_{\rho}=\{x|| x \mid<\rho\}$, and $\rho$ is a constant satisfying $\rho>1$. Equation (4.1.1) is then a special case of (4.2.15) with $\epsilon=1$.

The highlights of our method, as shown in the sequel, is based on the following.

a) Repeated iterations of (4.2.16) as manifested in (4.2.17).

b) Change order of summations in multi-sums as manifested in (4.2.18).

c) Combine a) and b) to obtain a new equation for the unknown $\hat{Z}(t, \epsilon)$.

d) Show that the unique solution of the equation in c) is also a solution of the original equation (4.2.16).

Consider the corresponding difference system

$$
\hat{Z}(t, \epsilon)=I-\sum_{t_{1}=t}^{b} \epsilon \widetilde{R}\left(t_{1}\right) \hat{Z}\left(t_{1}, \epsilon\right)=I+\epsilon \mathcal{L} \hat{Z}(t, \epsilon)
$$

If $\hat{Z}(t, \epsilon)$ is a solution of $\hat{Z}=I+\epsilon \mathcal{L} \hat{Z}$, then

$$
\begin{aligned}
\hat{Z} & =I+\epsilon \mathcal{L} \hat{Z} \\
& =I+\epsilon \mathcal{L}(I+\epsilon \mathcal{L} \hat{Z}) \\
& =I+\epsilon \mathcal{L} I+\epsilon^{2} \mathcal{L}^{2} I \\
& =I+\epsilon \mathcal{L} I+\cdots \epsilon^{m-1} \mathcal{L}^{m-1} I+\epsilon^{m} \mathcal{L}^{m} \hat{Z}
\end{aligned}
$$

where $m \in \mathbb{N}$ and $m \geq 2$.

It can be easily verified that for any $m \in \mathbb{N}$ and $m \geq 2$,

$$
\mathcal{L}^{m} \hat{Z}(t, \epsilon)=(-1)^{m} \sum_{t_{m}=t}^{b} \sum_{t_{m-1}=t_{m}}^{b} \cdots \sum_{t_{1}=t_{2}}^{b} \widetilde{R}\left(t_{m}\right) \widetilde{R}\left(t_{m-1}\right) \cdots \widetilde{R}\left(t_{1}\right) \hat{Z}\left(t_{1}, \epsilon\right)
$$


By Lemma 4.2.2, we have

$$
\begin{aligned}
\mathcal{L}^{m} \hat{Z}(t, \epsilon)= & (-1)^{m} \sum_{t_{1}=t}^{b} \sum_{t_{2}=t}^{t_{1}} \sum_{t_{3}=t}^{t_{2}} \cdots \sum_{t_{m}=t}^{t_{m-1}} \widetilde{R}\left(t_{m}\right) \widetilde{R}\left(t_{m-1}\right) \cdots \widetilde{R}\left(t_{1}\right) \hat{Z}\left(t_{1}, \epsilon\right) \\
= & (-1)^{m} \sum_{t_{1}=t}^{b} \sum_{t_{2}=t}^{b} \sum_{t_{3}=t}^{t_{2}} \cdots \sum_{t_{m}=t}^{t_{m-1}} \widetilde{R}\left(t_{m}\right) \widetilde{R}\left(t_{m-1}\right) \cdots \widetilde{R}\left(t_{1}\right) \hat{Z}\left(t_{1}, \epsilon\right) \\
& -(-1)^{m} \sum_{t_{1}=t}^{b} \sum_{t_{2}=t_{1}+1}^{b} \sum_{t_{3}=t}^{t_{2}} \cdots \sum_{t_{m}=t}^{t_{m-1}} \widetilde{R}\left(t_{m}\right) \widetilde{R}\left(t_{m-1}\right) \cdots \widetilde{R}\left(t_{1}\right) \hat{Z}\left(t_{1}, \epsilon\right) \\
= & {\left[-\sum_{t_{1}=t}^{b} \widetilde{R}\left(t_{1}\right) \hat{Z}\left(t_{1}, \epsilon\right)\right] \cdot(-1)^{m-1} \sum_{t_{2}=t}^{b} \sum_{t_{3}=t}^{t_{2}} \cdots \sum_{t_{m}=t}^{t_{m-1}} \widetilde{R}\left(t_{m}\right) \widetilde{R}\left(t_{m-1}\right) \cdots \widetilde{R}\left(t_{2}\right) } \\
& -(-1)^{m} \sum_{t_{1}=t}^{b} C\left(t_{1}, t\right) \widetilde{R}\left(t_{1}\right) \hat{Z}\left(t_{1}, \epsilon\right) \\
= & \mathcal{L} \hat{Z} \cdot \mathcal{L}^{m-1} I-(-1)^{m} \sum_{t_{1}=t}^{b} C\left(t_{1}, t\right) \widetilde{R}\left(t_{1}\right) \hat{Z}\left(t_{1}, \epsilon\right),
\end{aligned}
$$

where $C\left(t_{1}, t\right)$ is defined in (4.2.8).

Here we get a useful identity for a later discussion,

$$
-(-1)^{m} \sum_{t_{1}=t}^{b} C\left(t_{1}, t\right) \widetilde{R}\left(t_{1}\right) \hat{Z}\left(t_{1}, \epsilon\right)=\mathcal{L}^{m} \hat{Z}(t, \epsilon)-\mathcal{L} \hat{Z}(t, \epsilon) \cdot \mathcal{L}^{m-1} I .
$$

This identity holds for any $\hat{Z}(t, \epsilon)$ and is not limited to solutions of (4.2.16) or (4.2.17).

$>$ From (4.2.17) and (4.2.18), we get

$$
\begin{aligned}
& \hat{Z}=I+\epsilon \mathcal{L} I+\cdots \epsilon^{m-1} \mathcal{L}^{m-1} I+\epsilon^{m} \mathcal{L}^{m} \hat{Z} \\
= & I+\epsilon \mathcal{L} I+\cdots \epsilon^{m-1} \mathcal{L}^{m-1} I+\epsilon^{m}\left(\mathcal{L} \hat{Z} \cdot \mathcal{L}^{m-1} I-(-1)^{m} \sum_{t_{1}=t}^{b} C\left(t_{1}, t\right) \widetilde{R}\left(t_{1}\right) \hat{Z}\left(t_{1}, \epsilon\right)\right) \\
= & I+\epsilon \mathcal{L} I+\cdots \epsilon^{m-1} \mathcal{L}^{m-1} I+\epsilon^{m}\left(\frac{\hat{Z}-I}{\epsilon}\left(\mathcal{L}^{m-1} I\right)-(-1)^{m} \sum_{t_{1}=t}^{b} C\left(t_{1}, t\right) \widetilde{R}\left(t_{1}\right) \hat{Z}\left(t_{1}, \epsilon\right)\right) \\
= & I+\epsilon \mathcal{L} I+\cdots \epsilon^{m-2} \mathcal{L}^{m-2} I+\epsilon^{m-1}\left(\mathcal{L}^{m-1} I\right) \hat{Z}-\epsilon^{m}(-1)^{m} \sum_{t_{1}=t}^{b} C\left(t_{1}, t\right) \widetilde{R}\left(t_{1}\right) \hat{Z}\left(t_{1}, \epsilon\right),
\end{aligned}
$$

which leads to

$$
\left(I-\epsilon^{m-1} \mathcal{L}^{m-1} I\right) \hat{Z}=I+\epsilon \mathcal{L} I+\cdots \epsilon^{m-2} \mathcal{L}^{m-2} I-\epsilon^{m}(-1)^{m} \sum_{t_{1}=t}^{b} C\left(t_{1}, t\right) \widetilde{R}\left(t_{1}\right) \hat{Z}\left(t_{1}, \epsilon\right) .
$$

Thus, we get a new equation

$$
\hat{Z}(t, \epsilon)=\left(I-\epsilon^{m-1} \mathcal{L}^{m-1} I\right)^{-1}\left[\sum_{\nu=0}^{m-2} \epsilon^{\nu} \mathcal{L}^{\nu} I-\epsilon^{m}(-1)^{m} \sum_{t_{1}=t}^{b} C\left(t_{1}, t\right) \widetilde{R}\left(t_{1}\right) \hat{Z}\left(t_{1}, \epsilon\right)\right],
$$


provided that $\left(I-\epsilon^{m-1} \mathcal{L}^{m-1} I\right)^{-1}$ exists.

Equation (4.2.20) can be rewritten as

$$
\hat{Z}=\hat{H}+\mathcal{P} \hat{Z}
$$

where

$$
\begin{aligned}
\hat{H} & :=\left(I-\epsilon^{m-1} \mathcal{L}^{m-1} I\right)^{-1} \sum_{\nu=0}^{m-2} \epsilon^{\nu} \mathcal{L}^{\nu} I, \\
\mathcal{P} \hat{Z}(t, \epsilon) & :=-\left(I-\epsilon^{m-1} \mathcal{L}^{m-1} I\right)^{-1} \epsilon^{m}(-1)^{m} \sum_{t_{1}=t}^{b} C\left(t_{1}, t\right) \widetilde{R}\left(t_{1}\right) \hat{Z}\left(t_{1}, \epsilon\right) .
\end{aligned}
$$

For the convenience of the later discussion, we define

$$
\mathcal{L}^{0} I=I, \quad \mathcal{P}^{0} I=\hat{H} .
$$

For any bounded valued matrix function $A(t, \epsilon)$, we choose the norm

$$
\|A(t, \epsilon)\|=\sum_{j, k=1}^{n}\left|a_{j k}(t, \epsilon)\right|
$$

which leads of course to the useful relation

$$
\|A(t, \epsilon) B(t, \epsilon)\| \leq\|A(t, \epsilon)\| \cdot\|B(t, \epsilon)\| .
$$

Then we have the following lemma.

LEMMA 4.2.3. Assume that we have uniformly for $b \leq \infty$,

(i) $\mathcal{L}^{m-1} I$ is a convergent summation when $t \in[a, b)$,

(ii) $\quad \mathcal{L}^{m-1} I \rightarrow 0$ as $t \rightarrow b$, and

$$
\text { (iii) } \sum_{t_{1}=t}^{b}\left\|C\left(t_{1}, t\right) \tilde{R}\left(t_{1}\right)\right\| \rightarrow 0 \text { as } t \rightarrow b .
$$

Then, for $\epsilon \in D_{\rho}$ with $\rho>1$, we have the following conclusions.

(a) $\hat{X}(t, \epsilon):=\sum_{k=0}^{\infty} \mathcal{P}^{k} \hat{H}$ converges absolutely and uniformly when $t$ belongs to some interval $[a, b)$ and $a$ is large enough. $\hat{X}(t, \epsilon)$ is a unique solution of (4.2.21). Moreover,

(b) $\hat{X}(t, \epsilon)=\sum_{j=0}^{\infty}\left(\mathcal{L}^{j} I\right) \epsilon^{j}$, and $\triangle \hat{X}(t, \epsilon)=\epsilon \tilde{R}(t) \hat{X}(t, \epsilon)$.

Proof. (a) By the conditions (4.2.25), (4.2.27), and $\epsilon \in D_{\rho}$, there exists some $a \in \mathbb{R}$ with $a<b$, and $\delta \in(0,1)$ such that for any $t \in[a, b),\left(I-\epsilon^{m-1} \mathcal{L}^{m-1} I\right)^{-1}$ exists and

$$
\left\|\left(I-\epsilon^{m-1} \mathcal{L}^{m-1} I\right)^{-1}\right\| \cdot|\epsilon|^{m} \sum_{t_{1}=t}^{b}\left\|C\left(t_{1}, t\right) \widetilde{R}\left(t_{1}\right)\right\|<\delta .
$$

For each $\epsilon \in D_{\rho}$, we define when $t \in[a, b)$,

$$
\|A(t, \epsilon)\|\left\|=\sup _{t \in[a, b)}\right\| A(t, \epsilon) \| .
$$

We also define a series $\left\{\hat{Z}_{n}\right\}_{n=0,1,2 \ldots}$ by

$$
\hat{Z}_{0}=\hat{H}, \quad \hat{Z}_{j+1}=\hat{H}+\mathcal{P} \hat{Z}_{j}, \text { for } j=0,1,2, \cdots .
$$


For any $t \in[a, b), \epsilon \in D_{\rho}$, we have

$$
\begin{aligned}
& \left\|\hat{Z}_{j+1}-\hat{Z}_{j}\right\|=\left\|\left(\hat{H}+\mathcal{P} \hat{Z}_{j}\right)-\left(\hat{H}+\mathcal{P} \hat{Z}_{j-1}\right)\right\|=\left\|\mathcal{P} \hat{Z}_{j}-\mathcal{P} \hat{Z}_{j-1}\right\| \\
= & \left\|-\left(I-\epsilon^{m-1} \mathcal{L}^{m-1} I\right)^{-1} \epsilon^{m}(-1)^{m} \sum_{t_{1}=t}^{b} C\left(t_{1}, t\right) \widetilde{R}\left(t_{1}\right)\left[\hat{Z}_{j}\left(t_{1}, \epsilon\right)-\hat{Z}_{j-1}\left(t_{1}, \epsilon\right)\right]\right\| \\
\leq & \left\|\left(I-\epsilon^{m-1} \mathcal{L}^{m-1} I\right)^{-1}\right\| \cdot|\epsilon|^{m} \sum_{t_{1}=t}^{b}\left(\left\|C\left(t_{1}, t\right) \widetilde{R}\left(t_{1}\right)\right\| \cdot\left\|\hat{Z}_{j}\left(t_{1}, \epsilon\right)-\hat{Z}_{j-1}\left(t_{1}, \epsilon\right)\right\|\right) \\
\leq & \left(\left(I-\epsilon^{m-1} \mathcal{L}^{m-1} I\right)^{-1}\left\|\cdot|\epsilon|^{m} \sum_{t_{1}=t}^{b}\right\| C\left(t_{1}, t\right) \widetilde{R}\left(t_{1}\right) \|\right)\left\|\hat{Z}_{j}(t, \epsilon)-\hat{Z}_{j-1}(t, \epsilon)\right\| \\
\leq & \delta\left\|\hat{Z}_{j}-\hat{Z}_{j-1}\right\| \| .
\end{aligned}
$$

Therefore, $\left\|\left|\hat{Z}_{j+1}-\hat{Z}_{j}\||\leq \delta|\| \hat{Z}_{j}-\hat{Z}_{j-1}\|\|\right.\right.$, which leads to the conclusion that $\left\{\hat{Z}_{n}\right\}$ converges uniformly for $\epsilon \in D_{\rho}$ and $t \in[a, b)$.

At the same time, notice

$$
\hat{Z}_{n}=\hat{H}+\mathcal{P} \hat{Z}_{n-1}=\hat{H}+\mathcal{P} \hat{H}+\mathcal{P}^{2} \hat{Z}_{n-2}=\hat{H}+\mathcal{P} \hat{H}+\cdots+\mathcal{P}^{n} \hat{H}
$$

so that

$$
\lim _{n \rightarrow \infty} \hat{Z}_{n}=\sum_{k=0}^{\infty} \mathcal{P}^{k} \hat{H}
$$

Thus, we conclude that $\sum_{k=0}^{\infty} \mathcal{P}^{k} \hat{H}$ converges absolutely and uniformly for $\epsilon \in D_{\rho}$ and $t \in[a, b)$. Observe that $\sum_{k=0}^{\infty} \mathcal{P}^{k} \hat{H}=\hat{H}+\mathcal{P}\left(\sum_{k=0}^{\infty} \mathcal{P}^{k} \hat{H}\right)$, we get that $\hat{X}(t, \epsilon)=\sum_{k=0}^{\infty} \mathcal{P}^{k} \hat{H}$ is a solution of (4.2.21).

Since $\|\mathcal{P}\| \leq\left\|\left(I-\epsilon^{m-1} \mathcal{L}^{m-1} I\right)^{-1}\right\| \cdot|\epsilon|^{m} \sum_{t_{1}=t}^{b}\left\|C\left(t_{1}, t\right) \widetilde{R}\left(t_{1}\right)\right\|<\delta<1$, we also have that $\hat{X}(t, \epsilon)$ is the unique solution of $(4.2 .21)$.

(b) Since $\hat{Z}_{0}(t, \epsilon)=\hat{H}=\left(I-\epsilon^{m-1} \mathcal{L}^{m-1} I\right)^{-1} \sum_{\nu=0}^{m-2} \epsilon^{\nu} \mathcal{L}^{\nu} I$, we get that $\hat{Z}_{0}(t, \epsilon)$ is an analytical matrix function of $\epsilon$ for $\epsilon \in D_{\rho}$, uniformly for $b \in(a, \infty]$.

By induction, as

$$
\hat{Z}_{j+1}=\hat{H}+\mathcal{P} \hat{Z}_{j}=\left(I-\epsilon^{m-1} \mathcal{L}^{m-1} I\right)^{-1}\left[\sum_{\nu=0}^{m-2} \epsilon^{\nu} \mathcal{L}^{\nu} I-\epsilon^{m}(-1)^{m} \sum_{t_{1}=t}^{b} C\left(t_{1}, t\right) \widetilde{R}\left(t_{1}\right) \hat{Z}_{j}\left(t_{1}, \epsilon\right)\right]
$$

each $\hat{Z}_{j}(t, \epsilon)$ is an analytical matrix function of $\epsilon$ for $\epsilon \in D_{\rho}$, uniformly for $b \in(a, \infty]$.

Now, since $\hat{Z}_{j}(t, \epsilon) \rightarrow \hat{X}(t, \epsilon)$, uniformly for $t \in(a, b], b \in(a, \infty]$ and $\epsilon \in D_{\rho}$, as $j \rightarrow \infty$, we conclude that $\hat{X}(t, \epsilon)$ is an analytical matrix function of $\epsilon$ for $\epsilon \in D_{\rho}$. Therefore, we can express $\hat{X}(t, \epsilon)$ as an absolutely convergence power series of $\epsilon$ in $D_{\rho}$ with $\rho>1$. Namely,

$$
\hat{X}(t, \epsilon)=\sum_{j=0}^{\infty} \hat{A}_{j}(t) \epsilon^{j}
$$


Let us find the coefficients $\hat{A}_{j}(t)$. Since $\hat{X}(t, \epsilon)$ is a solution of equation (4.2.21), it is also a solution of

$$
\left(I-\epsilon^{m-1} \mathcal{L}^{m-1} I\right) \hat{Z}(t, \epsilon)=\sum_{\nu=0}^{m-2} \epsilon^{\nu} \mathcal{L}^{\nu} I-\epsilon^{m}(-1)^{m} \sum_{t_{1}=t}^{b} C\left(t_{1}, t\right) \widetilde{R}\left(t_{1}\right) \hat{Z}\left(t_{1}, \epsilon\right) .
$$

Use the identity (4.2.19) in (4.2.30) to get that $\hat{X}(t, \epsilon)$ solves the equation

$$
\left(I-\epsilon^{m-1} \mathcal{L}^{m-1} I\right) \hat{X}(t, \epsilon)=\sum_{\nu=0}^{m-2} \epsilon^{\nu} \mathcal{L}^{\nu} I+\epsilon^{m}\left(\mathcal{L}^{m} \hat{X}(t, \epsilon)-\mathcal{L} \hat{X}(t, \epsilon) \cdot \mathcal{L}^{m-1} I\right),
$$

which leads to

$$
\begin{aligned}
\hat{X}(t, \epsilon) & =\sum_{\nu=0}^{m-2} \epsilon^{\nu} \mathcal{L}^{\nu} I+\epsilon^{m}\left(\mathcal{L}^{m} \hat{X}(t, \epsilon)-\mathcal{L} \hat{X}(t, \epsilon) \cdot \mathcal{L}^{m-1} I\right)+\epsilon^{m-1} \mathcal{L}^{m-1} I \cdot \hat{X}(t, \epsilon) \\
& =\sum_{\nu=0}^{m-2} \epsilon^{\nu} \mathcal{L}^{\nu} I+\epsilon^{m-1} \mathcal{L}^{m-1} I(\hat{X}(t, \epsilon)-\epsilon \mathcal{L} \hat{X}(t, \epsilon))+\epsilon^{m} \mathcal{L}^{m} \hat{X}(t, \epsilon)
\end{aligned}
$$

Observe that, by (4.2.29),

$$
\begin{aligned}
\hat{X}(t, \epsilon) & =\sum_{j=0}^{\infty} \hat{A}_{j}(t) \epsilon^{j}=\hat{A}_{0}(t)+\sum_{j=0}^{\infty} \hat{A}_{j+1}(t) \epsilon^{j+1}, \\
\epsilon \mathcal{L} \hat{X}(t, \epsilon) & =\epsilon \mathcal{L}\left(\sum_{j=0}^{\infty} \hat{A}_{j}(t) \epsilon^{j}\right)=\sum_{j=0}^{\infty}\left(\mathcal{L} \hat{A}_{j}(t) \epsilon^{j+1}\right) .
\end{aligned}
$$

Therefore,

$$
\begin{aligned}
& \epsilon^{m-1} \mathcal{L}^{m-1} I(\hat{X}(t, \epsilon)-\epsilon \mathcal{L} \hat{X}(t, \epsilon)) \\
= & \epsilon^{m-1} \mathcal{L}^{m-1} I\left(\hat{A}_{0}(t)+\sum_{j=0}^{\infty} \hat{A}_{j+1}(t) \epsilon^{j+1}-\sum_{j=0}^{\infty}\left(\mathcal{L} \hat{A}_{j}(t) \epsilon^{j+1}\right)\right) \\
= & \left(\mathcal{L}^{m-1} I\right) \hat{A}_{0}(t) \epsilon^{m-1}+\sum_{j=0}^{\infty}\left(\mathcal{L}^{m-1} I\right)\left(\hat{A}_{j+1}(t)-\mathcal{L} \hat{A}_{j}(t)\right) \epsilon^{m+j} .
\end{aligned}
$$

At the same time,

$$
\epsilon^{m} \mathcal{L}^{m} \hat{X}(t, \epsilon)=\epsilon^{m} \mathcal{L}^{m}\left(\sum_{j=0}^{\infty} \hat{A}_{j}(t) \epsilon^{j}\right)=\sum_{j=0}^{\infty}\left(\mathcal{L}^{m} \hat{A}_{j}(t)\right) \epsilon^{m+j} .
$$

Hence, by (4.2.29), (4.2.31), (4.2.32) and (4.2.33), we get that

$$
\begin{aligned}
\sum_{j=0}^{\infty} \hat{A}_{j}(t) \epsilon^{j}= & \sum_{\nu=0}^{m-2} \mathcal{L}^{\nu} I \cdot \epsilon^{\nu}+\left(\mathcal{L}^{m-1} I\right) \hat{A}_{0}(t) \epsilon^{m-1} \\
& +\sum_{j=0}^{\infty}\left[\left(\mathcal{L}^{m-1} I\right)\left(\hat{A}_{j+1}(t)-\mathcal{L} \hat{A}_{j}(t)\right)+\mathcal{L}^{m} \hat{A}_{j}(t)\right] \epsilon^{m+j}
\end{aligned}
$$


By comparing the coefficients of $\epsilon^{0}, \epsilon^{1}, \cdots \epsilon^{m-2}$ on both sides of (4.2.34), we get that

$$
\hat{A}_{0}(t)=I, \quad \hat{A}_{1}(t)=\mathcal{L} I, \quad \cdots \quad, \hat{A}_{m-2}(t)=\mathcal{L}^{m-2} I .
$$

Also, comparing the coefficient of $\epsilon^{m-1}$ in (4.2.34), we get

$$
\hat{A}_{m-1}(t)=\left(\mathcal{L}^{m-1} I\right) \hat{A}_{0}(t)=\mathcal{L}^{m-1} I .
$$

At last, compare the coefficients of $\epsilon^{m+j}, j=0,1,2 \cdots$, in (4.2.34) to get

$$
\hat{A}_{m+j}(t)=\left(\mathcal{L}^{m-1} I\right)\left(\hat{A}_{j+1}(t)-\mathcal{L} \hat{A}_{j}(t)\right)+\mathcal{L}^{m} \hat{A}_{j}(t) .
$$

Then, by induction, it is easy to verify that

$$
\hat{A}_{m+j}(t)=\mathcal{L}^{m+j} I, \quad j=0,1,2 \cdots .
$$

Therefore, we get that $\hat{A}_{j}(t)=\mathcal{L}^{j} I, j=0,1,2 \cdots$, which means that

$$
\hat{X}(t, \epsilon)=\sum_{j=0}^{\infty}\left(\mathcal{L}^{j} I\right) \epsilon^{j}
$$

Notice that since $\sum_{j=0}^{\infty}\left(\mathcal{L}^{j} I\right) \epsilon^{j}=I+\epsilon \mathcal{L}\left(\sum_{j=0}^{\infty}\left(\mathcal{L}^{j} I\right) \epsilon^{j}\right)$, we get that $\hat{X}(t, \epsilon)$ is a solution of $\hat{Z}=I+\epsilon \mathcal{L} \hat{Z}$. Therefore,

$$
\triangle \hat{X}(t, \epsilon)=\epsilon \tilde{R}(t) \hat{X}(t, \epsilon)
$$

which is the desired conclusion.

We can now conclude the proof of Theorem 4.2.1.

Proof. When $\epsilon=1$, we have in (4.2.22) and (4.2.23)

$$
\begin{aligned}
\hat{H} & =\left(I-\mathcal{L}^{m-1} I\right)^{-1} \sum_{\nu=0}^{m-2} \mathcal{L}^{\nu} I, \\
\mathcal{P} \hat{Z}(t) & =-\left(I-\mathcal{L}^{m-1} I\right)^{-1}(-1)^{m} \sum_{t_{1}=t}^{b} C\left(t_{1}, t\right) \widetilde{R}\left(t_{1}\right) \hat{Z}\left(t_{1}\right) .
\end{aligned}
$$

$>$ From Lemma 4.2.3 we know that $\hat{X}(t)=\sum_{k=0}^{\infty} \mathcal{P}^{k} \hat{H}$ converges absolutely and uniformly, and $\triangle \hat{X}(t)=\tilde{R}(t) \hat{X}(t)$. Therefore,

$$
S(t)=\left(\prod_{l=a}^{t-1} D(l)\right) \hat{X}(t)
$$

is a fundamental solution of equation (4.1.1).

Also, in the proof of Lemma 4.2 .3 , we know that $\hat{X}(t)$ is a solution of $\hat{Z}=\hat{H}+\mathcal{P} \hat{Z}$. Therefore,

$$
\hat{X}=\left(I-\mathcal{L}^{m-1} I\right)^{-1} \sum_{\nu=0}^{m-2} \mathcal{L}^{\nu} I-\left(I-\mathcal{L}^{m-1} I\right)^{-1} \cdot\left[(-1)^{m} \sum_{t_{1}=t}^{b} C\left(t_{1}, t\right) \widetilde{R}\left(t_{1}\right) \hat{X}\left(t_{1}\right)\right] .
$$


We can estimate $\|\hat{X}-I\|$ as following,

$$
\begin{aligned}
& \quad\|\hat{X}-I\| \\
& =\left\|\left(I-\mathcal{L}^{m-1} I\right) \sum_{\nu=0}^{m-2} \mathcal{L}^{\nu} I-I-\left(I-\mathcal{L}^{m-1} I\right)^{-1} \cdot\left[(-1)^{m} \sum_{t_{1}=t}^{b} C\left(t_{1}, t\right) \widetilde{R}\left(t_{1}\right) \hat{X}\left(t_{1}\right)\right]\right\| \\
& \leq \quad\left\|\left(I-\mathcal{L}^{m-1} I\right)^{-1} \sum_{\nu=0}^{m-2} \mathcal{L}^{\nu} I-I\right\|+\left\|\left(I-\mathcal{L}^{m-1} I\right)^{-1}\right\| \cdot \sum_{t_{1}=t}^{b}\left\|C\left(t_{1}, t\right) \widetilde{R}\left(t_{1}\right) \hat{X}\left(t_{1}\right)\right\| \\
& \leq \quad\left\|\left(I-\mathcal{L}^{m-1} I\right)^{-1} \sum_{\nu=0}^{m-2} \mathcal{L}^{\nu} I-I\right\| \\
& \quad+\left\|\left(I-\mathcal{L}^{m-1} I\right)^{-1}\right\| \cdot\left(\sum_{t_{1}=t}^{b}\left\|C\left(t_{1}, t\right) \widetilde{R}\left(t_{1}\right)\right\|\right)\|\hat{X}\| \| .
\end{aligned}
$$

By the condition (4.2.9), we have $\mathcal{L}^{m-1} I \rightarrow 0$ and $\sum_{\nu=0}^{m-2} \mathcal{L}^{\nu} I \rightarrow I$ as $t \rightarrow b$. Hence, we get that

$$
\left\|\left(I-\mathcal{L}^{m-1} I\right)^{-1} \sum_{\nu=0}^{m-2} \mathcal{L}^{\nu} I-I\right\| \rightarrow 0 \text { as } t \rightarrow b .
$$

Moreover, ||$\hat{X}|| \mid$ is finite because $\sum_{k=0}^{\infty} \mathcal{P}^{k} \hat{H}$ converges absolutely and uniformly on $[a, b)$. $\left\|\left(I-\mathcal{L}^{m-1} I\right)^{-1}\right\| \rightarrow n$ as $t \rightarrow b . \sum_{t_{1}=t}^{b}\left\|C\left(t_{1}, t\right) \widetilde{R}\left(t_{1}\right)\right\| \rightarrow 0$ as $t \rightarrow b$ because of the condition (4.2.11). Thus, we know that

$$
\left\|\left(I-\mathcal{L}^{m-1} I\right)^{-1}\right\| \cdot\left(\sum_{t_{1}=t}^{b}\left\|C\left(t_{1}, t\right) \widetilde{R}\left(t_{1}\right)\right\|\right)\|\hat{X}\| \| \rightarrow 0 \text { as } t \rightarrow b .
$$

$>$ From (4.2.37), (4.2.38) and (4.2.39), we get that $\|\hat{X}-I\| \rightarrow 0$ as $t \rightarrow b$. Let $P(t):=$ $\hat{X}-I$, then $P(t) \rightarrow 0$ as $t \rightarrow b$. From (4.2.35), we also have that

$$
P(t)=\sum_{j=1}^{\infty} \mathcal{L}^{j} I
$$

Hence, by (4.2.36),

$$
S(t)=\left(\prod_{l=a}^{t-1} D(l)\right)(I+P(t))
$$

is a fundamental solution of equation (4.1.1) with $P(t) \rightarrow 0$ as $t \rightarrow b$.

Theorem 4.2.1 with $m=2$ and $b=\infty$ leads to a simple criterion for asymptotic summation that will be used frequently in the sequel. Therefore, we have the following. 
THEOREM 4.2.4. Let $m=2, b=\infty$ and assume,

$$
\begin{aligned}
& \text { (i) } \sum_{t_{1}=t}^{\infty} \widetilde{R}\left(t_{1}\right) \rightarrow 0 \text { as } t \rightarrow \infty \text {, and } \\
& \text { (ii) } \sum_{t_{1}=t}^{\infty}\left\|\left(\sum_{t_{2}=t_{1}+1}^{\infty} \widetilde{R}\left(t_{2}\right)\right) \widetilde{R}\left(t_{1}\right)\right\| \rightarrow 0 \text { as } t \rightarrow \infty .
\end{aligned}
$$

Then (4.1.1) is right almost diagonal.

Proof. When $m=2$ and $b=\infty$, we have $\mathcal{L} I=-\sum_{t_{1}=t}^{\infty} \widetilde{R}\left(t_{1}\right)$ and $C\left(t_{1}, t\right)=\sum_{t_{2}=t_{1}+1}^{\infty} \widetilde{R}\left(t_{2}\right)$. It is then easy to verify that all conditions of Theorem 4.2.1 hold and the result follows.

REMARK 4.2.5. Theorem 4.2.1 and Theorem 4.2.4 could apply to systems of difference equations that are not necessarily potentially oscillatory.

Our next result uses summation by parts to render the asymptotic summation of (4.1.1). For potentially oscillatory systems, it is more convenient to apply than Theorem 4.2 .4 because it imposed directly on $R(t)$ rather than on $\widetilde{R}(t)$.

THEOREM 4.2.6. Assume that (4.1.1) is potentially oscillatory and the following hold,

$$
\begin{array}{ll}
\text { (a) } & \sum_{t_{1}=t}^{\infty} R\left(t_{1}\right) \rightarrow 0 \text { as } t \rightarrow \infty \\
\text { (b) } & D^{-1}(t)\left[(I-D(t))\left(\sum_{t_{1}=t}^{\infty} R\left(t_{1}\right)\right)+\left(\sum_{t_{2}=t+1}^{\infty} R\left(t_{2}\right)\right)(D(t)-I)\right] \in l^{1}[a, \infty), \\
\text { (c) } & D^{-1}(t)\left(\sum_{t_{1}=t+1}^{\infty} R\left(t_{1}\right)\right) R(t) \in l^{1}[a, \infty) \\
\text { (d) } & \left\{\sum _ { t _ { 1 } = t + 1 } ^ { \infty } \left[\Phi ^ { - 1 } ( t _ { 1 } ) D ^ { - 1 } ( t _ { 1 } ) \left(\left(I-D\left(t_{1}\right)\right) \sum_{t_{2}=t_{1}}^{\infty} R\left(t_{2}\right)\right.\right.\right. \\
& \left.\left.\left.+\sum_{t_{3}=t_{1}+1}^{\infty} R\left(t_{3}\right)\left(D\left(t_{1}\right)-I\right)\right) \Phi\left(t_{1}\right)\right]\right\} \cdot \Phi^{-1}(t+1) R(t) \Phi(t) \in l^{1}[a, \infty) .
\end{array}
$$

Then (4.1.1) is right almost diagonal.

Proof. We first point out an identity of difference system that is useful for our discussion. For any matrix functions $A(t), B(t)$ and $C(t)$, when $t \leq b$, we have

$$
\begin{aligned}
& A(b+1) B(b+1) C(b+1)-A(t) B(t) C(t) \\
= & \sum_{t_{1}=t}^{b} \triangle\left[A\left(t_{1}\right) B\left(t_{1}\right) C\left(t_{1}\right)\right] \\
= & \sum_{t_{1}=t}^{b}\left[\left(\triangle A\left(t_{1}\right)\right) B\left(t_{1}\right) C\left(t_{1}\right)+A\left(t_{1}+1\right)\left(\triangle B\left(t_{1}\right)\right) C\left(t_{1}\right)+A\left(t_{1}+1\right) B\left(t_{1}+1\right)\left(\triangle C\left(t_{1}\right)\right)\right],
\end{aligned}
$$


which leads to

$$
\begin{aligned}
& \sum_{t_{1}=t}^{b} A\left(t_{1}+1\right)\left(\triangle B\left(t_{1}\right)\right) C\left(t_{1}\right) \\
= & A(b+1) B(b+1) C(b+1)-A(t) B(t) C(t) \\
& -\sum_{t_{1}=t}^{b}\left[\left(\triangle A\left(t_{1}\right)\right) B\left(t_{1}\right) C\left(t_{1}\right)+A\left(t_{1}+1\right) B\left(t_{1}+1\right)\left(\triangle C\left(t_{1}\right)\right)\right] .
\end{aligned}
$$

We let $b=\infty, A(t)=\Phi^{-1}(t), B(t)=-\sum_{t_{1}=t}^{\infty} R\left(t_{1}\right)$, and $C(t)=\Phi(t)$.

We now need the Proposition 1.2.3. By (1.2.8) and the condition (4.2.41) of this theorem, we get that

$$
\lim _{t \rightarrow \infty} \Phi^{-1}(t)\left(-\sum_{t_{1}=t}^{\infty} R\left(t_{1}\right)\right) \Phi(t)=0 .
$$

Hence, (4.2.45) implies that

$$
\begin{aligned}
& \sum_{t_{1}=t}^{b} \Phi^{-1}\left(t_{1}+1\right) R\left(t_{1}\right) \Phi\left(t_{1}\right) \\
= & \Phi^{-1}(t)\left[\sum_{t_{1}=t}^{\infty} R\left(t_{1}\right)\right] \Phi(t)+\sum_{t_{1}=t}^{b}\left[\triangle \Phi^{-1}\left(t_{1}\right) \sum_{t_{2}=t_{1}}^{\infty} R\left(t_{2}\right) \Phi\left(t_{1}\right)\right. \\
& \left.+\Phi^{-1}\left(t_{1}+1\right) \sum_{t_{3}=t_{1}+1}^{\infty} R\left(t_{3}\right) \triangle \Phi\left(t_{1}\right)\right] .
\end{aligned}
$$

$>$ From (4.2.2), we obtain that

$$
\triangle \Phi(t)=[D(t)-I] \Phi(t) .
$$

Also, (4.2.2) infer that $\Phi^{-1}(t)=\Phi^{-1}(t+1) D(t)$. Therefore,

$$
\triangle \Phi^{-1}(t)=\Phi^{-1}(t+1)-\Phi^{-1}(t)=\Phi^{-1}(t+1)[I-D(t)] .
$$

Put (4.2.48) and (4.2.49) into (4.2.47) and get that

$$
\begin{aligned}
& \sum_{t_{1}=t}^{\infty} \widetilde{R}\left(t_{1}\right)=\sum_{t_{1}=t}^{b} \Phi^{-1}\left(t_{1}+1\right) R\left(t_{1}\right) \Phi\left(t_{1}\right) \\
= & \Phi^{-1}(t) \sum_{t_{1}=t}^{\infty} R\left(t_{1}\right) \Phi(t) \\
& +\sum_{t_{1}=t}^{b}\left[\Phi^{-1}\left(t_{1}+1\right)\left(\left(I-D\left(t_{1}\right)\right) \sum_{t_{2}=t_{1}}^{\infty} R\left(t_{2}\right)+\sum_{t_{3}=t_{1}+1}^{\infty} R\left(t_{3}\right)\left(D\left(t_{1}\right)-I\right)\right) \Phi\left(t_{1}\right)\right] \\
= & \Phi^{-1}(t) \sum_{t_{1}=t}^{\infty} R\left(t_{1}\right) \Phi(t) \\
(4.2 .50)+ & +\sum_{t_{1}=t}^{b}\left[\Phi^{-1}\left(t_{1}\right) D^{-1}\left(t_{1}\right)\left(\left(I-D\left(t_{1}\right)\right) \sum_{t_{2}=t_{1}}^{\infty} R\left(t_{2}\right)+\sum_{t_{3}=t_{1}+1}^{\infty} R\left(t_{3}\right)\left(D\left(t_{1}\right)-I\right)\right) \Phi\left(t_{1}\right)\right] .
\end{aligned}
$$


Then, the conditions (4.2.41), (4.2.42) and the conclusions (1.2.8), (1.2.9) imply that $\sum_{t_{1}=t}^{\infty} \widetilde{R}\left(t_{1}\right) \rightarrow$ 0 as $t \rightarrow \infty$.

At last, the following identity that comes from (4.2.50) holds. Namely,

$$
\begin{gathered}
\left(\sum_{t_{1}=t+1}^{\infty} \widetilde{R}\left(t_{1}\right)\right) \widetilde{R}(t) \\
=\Phi^{-1}(t+1) \sum_{t_{1}=t+1}^{\infty} R\left(t_{1}\right) \Phi(t+1) \cdot \Phi^{-1}(t+1) R(t) \Phi(t) \\
+\sum_{t_{1}=t+1}^{b}\left[\Phi ^ { - 1 } ( t _ { 1 } ) D ^ { - 1 } ( t _ { 1 } ) \left(\left(I-D\left(t_{1}\right)\right) \sum_{t_{2}=t_{1}}^{\infty} R\left(t_{2}\right)\right.\right. \\
\left.\left.+\sum_{t_{3}=t_{1}+1}^{\infty} R\left(t_{3}\right)\left(D\left(t_{1}\right)-I\right)\right) \Phi\left(t_{1}\right)\right] \Phi^{-1}(t+1) R(t) \Phi(t) \\
=\Phi^{-1}(t) D^{-1}(t)\left(\sum_{t_{1}=t+1}^{\infty} R\left(t_{1}\right)\right) R(t) \Phi(t) \\
+\sum_{t_{1}=t+1}^{b}\left[\Phi ^ { - 1 } ( t _ { 1 } ) D ^ { - 1 } ( t _ { 1 } ) \left(\left(I-D\left(t_{1}\right)\right) \sum_{t_{2}=t_{1}}^{\infty} R\left(t_{2}\right)\right.\right. \\
\left.\left.\quad+\sum_{t_{3}=t_{1}+1}^{\infty} R\left(t_{3}\right)\left(D\left(t_{1}\right)-I\right)\right) \Phi\left(t_{1}\right)\right] \Phi(t+1) R(t) \Phi(t) .
\end{gathered}
$$

Thus, conditions (4.2.43), (4.2.44) and the conclusion (1.2.9) imply that

$$
\left(\sum_{t_{1}=t+1}^{\infty} \widetilde{R}\left(t_{1}\right)\right) \widetilde{R}(t) \in l^{1}[a, \infty) .
$$

Hence, by Theorem 4.2.4, (4.1.1) is right almost diagonal.

\subsection{Examples}

We will consider some examples of potentially oscillatory systems where $R(t)$ possesses elements of the form $(\sin t) \cdot t^{\underline{\delta}}$. Here, $t^{\underline{\delta}}$, with $\delta$ be any real number, is the "falling factorial power", which has the definition as in (2.3.21) of Chapter 2. Here we list some of its useful propositions as in the following.

First,

$$
\triangle t^{\underline{\delta}}=\delta t^{\underline{\delta-1}}
$$

Second,

$$
t^{\underline{\delta}} \sim t^{\delta}, \quad(t \rightarrow \infty)
$$

that is already proved in Chapter 2.

Third,

$$
t \frac{\delta-1}{t-\delta+1} t^{\underline{\delta}}
$$


which can be verified from the definition of $t^{\underline{\delta}}$ in $(2.3 .21)$.

Forth, when $\delta<0$ and $t \in[1, \infty)$,

$$
(t+1)^{\underline{\delta}}<t^{\underline{\delta}}
$$

Indeed, $t^{\underline{\delta}}$ and $(t+1)^{\underline{\delta}}$ are both positive numbers that can be verified from the definition (2.3.21). Thus,

$$
\begin{aligned}
\frac{(t+1)^{\underline{\delta}}}{t^{\underline{\delta}}} & =\frac{\Gamma(t+2)}{\Gamma(t-\delta+2)} \cdot \frac{\Gamma(t-\delta+1)}{\Gamma(t+1)} \\
& =\frac{(t+1) \Gamma(t+1)}{(t-\delta+1) \Gamma(t-\delta+1)} \cdot \frac{\Gamma(t-\delta+1)}{\Gamma(t+1)} \\
& =\frac{(t+1)}{(t-\delta+1)} \\
& <1
\end{aligned}
$$

which leads to (4.3.4).

Also, the following identity of summation by parts will be useful in our estimation. For any $a(t), c(t)$, with $t \leq b$,

$$
\sum_{t_{1}=t}^{b}\left[a\left(t_{1}\right) \triangle c\left(t_{1}\right)\right]=a(b+1) c(b+1)-a(t) c(t)-\sum_{t_{1}=t}^{b}\left[c\left(t_{1}+1\right) \triangle a\left(t_{1}\right)\right] .
$$

Indeed, we know that, see, e.g. [28],

$$
\sum[a(t) \triangle c(t)]=a(t) c(t)-\sum[c(t+1) \triangle a(t)]
$$

in which $\sum$ means the indefinite sum or antidifference operator. For any $Z(t),-\sum_{t_{1}=t}^{b} Z(t)$ is an indefinite sum. Hence, from (4.3.6), we can get

$$
-\sum_{t_{1}=t}^{b}[a(t) \triangle c(t)]=a(t) c(t)+\sum_{t_{1}=t}^{b}[c(t+1) \triangle a(t)]+C
$$

for some constant $C$. When $t=b$, we obtain $C=-a(b+1) c(b+1)$. Insert $C$ into (4.3.7), then (4.3.5) follows.

For any matrix function $A(t)$, the notation $[A(t)]_{t_{1}}^{t_{2}}$ means

$$
[A(t)]_{t_{1}}^{t_{2}}:=A\left(t_{2}\right)-A\left(t_{1}\right)
$$

Example 4.3.1. Consider a difference system on the interval $[1, \infty)$,

$$
Y(t+1)=\left(\left[\begin{array}{cccc}
\lambda_{1}(t) & 0 & \cdots & 0 \\
0 & \lambda_{2}(t) & \cdots & 0 \\
& \cdots & & \\
0 & 0 & \cdots & \lambda_{n}(t)
\end{array}\right]+\left[\begin{array}{cccc}
0 & c_{12} & \cdots & c_{1 n} \\
c_{21} & 0 & \cdots & c_{2 n} \\
& \cdots & & \\
c_{n 1} & c_{n 2} & \cdots & 0
\end{array}\right](\sin t) \cdot t^{\underline{\delta}}\right) Y(t) .
$$


If the following conditions hold,

(1) The system is potentially oscillatory and $\left|\lambda_{j}(t)\right| \geq \mu>0$, where $\mu$ is some positive constant;

$$
\begin{aligned}
& \text { (2) } \delta<-\frac{1}{2},\left|\frac{\lambda_{k}(t)}{\lambda_{j}(t+1)}-1\right|<M, \frac{1}{t-\delta+1}\left(\frac{\lambda_{k}(t)}{\lambda_{j}(t+1)}-1\right) \in l^{1}[1, \infty), \\
& \quad \triangle\left(\frac{\lambda_{k}(t)}{\lambda_{j}(t+1)}-1\right) \in l^{1}[1, \infty),\left(\frac{\lambda_{k}(t+1)}{\lambda_{j}(t+2)}-1\right)\left(\frac{\lambda_{k}(t)}{\lambda_{j}(t+1)}-1\right) \in l^{1}[1, \infty), \\
& \text { where } M>0 \text { is some constant, } j, k=1,2, \cdots n, \text { and } j \neq k .
\end{aligned}
$$

Then (4.3.9) is right almost diagonal.

ProOF. We want to apply Theorem 4.2.4. In this example,

$$
\widetilde{R}(t)=\left(\left(c_{j k}(\sin t) \cdot t^{\underline{\delta}}\right) \frac{\prod_{l=1}^{t-1} \lambda_{k}(l)}{\prod_{l=1}^{t} \lambda_{j}(l)}\right)_{j, k=1}^{n}
$$

Therefore,

$$
\begin{aligned}
\left(\sum_{t_{1}=t}^{\infty} \widetilde{R}\left(t_{1}\right)\right)_{j k}= & \sum_{t_{1}=t}^{\infty}\left(c_{j k}\left(\sin t_{1}\right) \cdot t_{1}^{\frac{\delta}{1}}\right) \cdot \frac{\prod_{l=1}^{t_{1}-1} \lambda_{k}(l)}{\prod_{l=1}^{t_{1}} \lambda_{j}(l)} \\
= & c_{j k}\left\{\left[\left(-\sum_{t_{2}=t_{1}}^{\infty}\left(\sin t_{2}\right) \cdot t_{2}^{\frac{\delta}{2}}\right) \frac{\prod_{l=1}^{t_{1}-1} \lambda_{k}(l)}{\prod_{l=1}^{t_{1}} \lambda_{j}(l)}\right]_{t}^{\infty}\right. \\
& \left.-\sum_{t_{1}=t}^{\infty}\left(-\sum_{t_{2}=t_{1}+1}^{\infty}\left(\sin t_{2}\right) \cdot t_{2}^{\frac{\delta}{2}}\right) \triangle \frac{\prod_{l=1}^{t_{1}-1} \lambda_{k}(l)}{\prod_{l=1}^{t_{1}} \lambda_{j}(l)}\right\} \\
= & c_{j k}\left\{\left(\sum_{t_{1}=t}^{\infty}\left(\sin t_{1}\right) \cdot t_{1}^{\frac{\delta}{1}}\right) \frac{\prod_{l=1}^{t-1} \lambda_{k}(l)}{\prod_{l=1}^{t} \lambda_{j}(l)}\right. \\
& \left.+\sum_{t_{1}=t}^{\infty}\left(\sum_{t_{2}=t_{1}+1}^{\infty}\left(\sin t_{2}\right) \cdot t_{2}^{\frac{\delta}{2}}\right)\left(\frac{\lambda_{k}\left(t_{1}\right)}{\lambda_{j}\left(t_{1}+1\right)}-1\right) \frac{\prod_{l=1}^{t_{1}-1} \lambda_{k}(l)}{\prod_{l=1}^{t_{1}} \lambda_{j}(l)}\right\} \\
= & c_{j k}\left\{A_{1}+A_{2}\right\}
\end{aligned}
$$

where

$$
\begin{aligned}
& A_{1}=\left(\sum_{t_{1}=t}^{\infty}\left(\sin t_{1}\right) \cdot t_{1}^{\frac{\delta}{1}}\right) \frac{\prod_{l=1}^{t-1} \lambda_{k}(l)}{\prod_{l=1}^{t} \lambda_{j}(l)} \\
& A_{2}=\sum_{t_{1}=t}^{\infty}\left(\sum_{t_{2}=t_{1}+1}^{\infty}\left(\sin t_{2}\right) \cdot t_{2}^{\frac{\delta}{2}}\right)\left(\frac{\lambda_{k}\left(t_{1}\right)}{\lambda_{j}\left(t_{1}+1\right)}-1\right) \frac{\prod_{l=1}^{t_{1}-1} \lambda_{k}(l)}{\prod_{l=1}^{t_{1}} \lambda_{j}(l)} .
\end{aligned}
$$


Now we estimate $A_{1}$. Observe that, using summation by parts,

$$
\begin{aligned}
\sum_{t_{1}=t}^{\infty}\left(\sin t_{1}\right) \cdot t_{1}^{\delta} & =\left[-\frac{\cos \left(t_{1}-\frac{1}{2}\right)}{2 \sin \frac{1}{2}} \cdot t_{1}^{\frac{\delta}{1}}\right]_{t}^{\infty}-\sum_{t_{1}=t}^{\infty}\left(-\frac{\cos \left(t_{1}+\frac{1}{2}\right)}{2 \sin \frac{1}{2}}\right) \Delta t_{1}^{\frac{\delta}{1}} \\
& =\frac{\cos \left(t-\frac{1}{2}\right)}{2 \sin \frac{1}{2}} \cdot t^{\frac{\delta}{2}}+\sum_{t_{1}=t}^{\infty}\left(\frac{\cos \left(t_{1}+\frac{1}{2}\right)}{2 \sin \frac{1}{2}}\right) \delta \cdot t_{1}^{\frac{\delta-1}{1}} .
\end{aligned}
$$

Therefore,

$$
\begin{aligned}
\left|\sum_{t_{1}=t}^{\infty}\left(\sin t_{1}\right) \cdot t_{1}^{\frac{\delta}{1}}\right| & \leq \frac{1}{2 \sin \frac{1}{2}} \cdot t^{\underline{\delta}}+\frac{1}{2 \sin \frac{1}{2}} \sum_{t_{1}=t}^{\infty}\left|\delta \cdot t_{1}^{\frac{\delta-1}{1}}\right| \\
& =\frac{1}{2 \sin \frac{1}{2}} \cdot t^{\underline{\delta}}+\frac{1}{2 \sin \frac{1}{2}}\left(-\sum_{t_{1}=t}^{\infty} \delta \cdot t^{\frac{\delta-1}{1}}\right) \\
& =\frac{1}{2 \sin \frac{1}{2}} \cdot t^{\underline{\delta}}+\frac{1}{2 \sin \frac{1}{2}} \cdot t^{\underline{\delta}} \\
& =M_{1} t^{\underline{\delta}}
\end{aligned}
$$

where $M_{1}=\frac{1}{\sin \frac{1}{2}}>0$.

Since the system (4.3.9) is potentially oscillatory and $\left|\lambda_{j}(t)\right| \geq \mu>0$, we can get that for all $t \in[1, \infty)$,

$$
\left|\frac{\prod_{l=1}^{t-1} \lambda_{k}(l)}{\prod_{l=1}^{t} \lambda_{j}(l)}\right|<M_{2},
$$

for some $M_{2}>0$. Hence, by (4.3.17) and (4.3.18), we have

$$
\left|A_{1}\right|=\left|\sum_{t_{1}=t}^{\infty}\left(\sin t_{1}\right) \cdot t_{1}^{\delta}\right| \cdot\left|\frac{\prod_{l=1}^{t-1} \lambda_{k}(l)}{\prod_{l=1}^{t} \lambda_{j}(l)}\right| \leq M_{1} M_{2} t^{\underline{\delta}} .
$$

Now consider $A_{2}$, we use summation by parts again in the right hand side of (4.3.16) to get

$$
\begin{aligned}
& \sum_{t_{1}=t}^{\infty}\left(\sin t_{1}\right) \cdot t_{1}^{\frac{\delta}{1}}=\frac{\cos \left(t-\frac{1}{2}\right)}{2 \sin \frac{1}{2}} \cdot t^{\underline{\delta}}+\frac{\delta}{2 \sin \frac{1}{2}} \sum_{t_{1}=t}^{\infty}\left[\cos \left(t_{1}+\frac{1}{2}\right)\right] \cdot t_{1}^{\frac{\delta-1}{1}} \\
& =\frac{\cos \left(t-\frac{1}{2}\right)}{2 \sin \frac{1}{2}} \cdot t^{\underline{\delta}}+\frac{\delta}{2 \sin \frac{1}{2}}\left\{\left[\frac{\sin t_{1}}{2 \sin \frac{1}{2}} \cdot t_{1}^{\delta-1}\right]_{t}^{\infty}-\sum_{t_{1}=t}^{\infty} \frac{\sin \left(t_{1}+1\right)}{2 \sin \frac{1}{2}} \cdot \Delta t_{1} \frac{\delta-1}{\delta}\right\} \\
& =\frac{\cos \left(t-\frac{1}{2}\right)}{2 \sin \frac{1}{2}} \cdot t^{\underline{\delta}}-\frac{\delta}{4\left(\sin \frac{1}{2}\right)^{2}}(\sin t) \cdot t^{\frac{\delta-1}{}} \\
& -\frac{\delta}{4\left(\sin \frac{1}{2}\right)^{2}} \sum_{t_{1}=t}^{\infty}\left[\sin \left(t_{1}+1\right)\right] \cdot(\delta-1) t \frac{\delta-2}{1} \text {. }
\end{aligned}
$$

By (4.3.15) and (4.3.20), we get that

$$
\left|A_{2}\right| \leq\left|F_{1}\right|+\left|F_{2}\right|+\left|F_{3}\right|
$$


where

$$
\begin{aligned}
F_{1}:= & \sum_{t_{1}=t}^{\infty} \frac{\cos \left(t_{1}+\frac{1}{2}\right)}{2 \sin \frac{1}{2}} \cdot\left(t_{1}+1\right)^{\underline{\delta}}\left(\frac{\lambda_{k}\left(t_{1}\right)}{\lambda_{j}\left(t_{1}+1\right)}-1\right) \frac{\prod_{l=1}^{t_{1}-1} \lambda_{k}(l)}{\prod_{l=1}^{t_{1}} \lambda_{j}(l)} \\
F_{2}:= & \sum_{t_{1}=t}^{\infty} \frac{\delta}{4\left(\sin \frac{1}{2}\right)^{2}}\left[\sin \left(t_{1}+1\right)\right] \cdot\left(t_{1}+1\right) \frac{\delta-1}{2}\left(\frac{\lambda_{k}\left(t_{1}\right)}{\lambda_{j}\left(t_{1}+1\right)}-1\right) \frac{\prod_{l=1}^{t_{1}-1} \lambda_{k}(l)}{\prod_{l=1}^{t_{1}} \lambda_{j}(l)} \\
F_{3}:= & \sum_{t_{1}=t}^{\infty}\left\{\frac{\delta}{4\left(\sin \frac{1}{2}\right)^{2}}\left(\sum_{t_{2}=t_{1}+1}^{\infty}\left[\sin \left(t_{2}+1\right)\right] \cdot(\delta-1) t_{2}^{\delta-2}\right)\right. \\
& \left.\left(\frac{\lambda_{k}\left(t_{1}\right)}{\lambda_{j}\left(t_{1}+1\right)}-1\right) \frac{\prod_{l=1}^{t_{1}-1} \lambda_{k}(l)}{\prod_{l=1}^{t_{1}} \lambda_{j}(l)} .\right\}
\end{aligned}
$$

For $F_{1}$ in $(4.3 .21)$, we get that using summation by parts

$$
\begin{aligned}
& \text { (4.3.23) }\left(2 \sin \frac{1}{2}\right) \cdot F_{1} \\
& =\sum_{t_{1}=t}^{\infty}\left[\cos \left(t_{1}+\frac{1}{2}\right)\right] \cdot\left(t_{1}+1\right)^{\underline{\delta}}\left(\frac{\lambda_{k}\left(t_{1}\right)}{\lambda_{j}\left(t_{1}+1\right)}-1\right) \frac{\prod_{l=1}^{t_{1}-1} \lambda_{k}(l)}{\prod_{l=1}^{t_{1}} \lambda_{j}(l)}
\end{aligned}
$$$$
=\left[\left(-\sum_{t_{2}=t_{1}}^{\infty}\left[\cos \left(t_{2}+\frac{1}{2}\right)\right] \cdot\left(t_{2}+1\right)^{\underline{\delta}}\right)\left(\frac{\lambda_{k}\left(t_{1}\right)}{\lambda_{j}\left(t_{1}+1\right)}-1\right) \frac{\prod_{l=1}^{t_{1}-1} \lambda_{k}(l)}{\prod_{l=1}^{t_{1}} \lambda_{j}(l)}\right]_{t}^{\infty}
$$$$
-\sum_{t_{1}=t}^{\infty}\left(-\sum_{t_{2}=t_{1}+1}^{\infty}\left[\cos \left(t_{2}+\frac{1}{2}\right)\right] \cdot\left(t_{2}+1\right)^{\underline{\delta}}\right) \triangle\left\{\left(\frac{\lambda_{k}\left(t_{1}\right)}{\lambda_{j}\left(t_{1}+1\right)}-1\right) \frac{\prod_{l=1}^{t_{1}-1} \lambda_{k}(l)}{\prod_{l=1}^{t_{1}} \lambda_{j}(l)}\right\}
$$$$
=\left(\sum_{t_{1}=t}^{\infty}\left[\cos \left(t_{1}+\frac{1}{2}\right)\right] \cdot\left(t_{1}+1\right)^{\underline{\delta}}\right)\left(\frac{\lambda_{k}(t)}{\lambda_{j}(t+1)}-1\right) \frac{\prod_{l=1}^{t-1} \lambda_{k}(l)}{\prod_{l=1}^{t} \lambda_{j}(l)}
$$

$$
\begin{aligned}
& +\sum_{t_{1}=t}^{\infty}\left(\sum_{t_{2}=t_{1}+1}^{\infty}\left[\cos \left(t_{2}+\frac{1}{2}\right)\right] \cdot\left(t_{2}+1\right)^{\underline{\delta}}\right)\left[\Delta\left(\frac{\lambda_{k}\left(t_{1}\right)}{\lambda_{j}\left(t_{1}+1\right)}-1\right)\right] \frac{\prod_{l=1}^{t_{1}-1} \lambda_{k}(l)}{\prod_{l=1}^{t_{1}} \lambda_{j}(l)} \\
& +\sum_{t_{1}=t}^{\infty}\left(\sum_{t_{2}=t_{1}+1}^{\infty}\left[\cos \left(t_{2}+\frac{1}{2}\right)\right] \cdot\left(t_{2}+1\right)^{\underline{\delta}}\right)\left(\frac{\lambda_{k}\left(t_{1}+1\right)}{\lambda_{j}\left(t_{1}+2\right)}-1\right)\left(\frac{\lambda_{k}\left(t_{1}\right)}{\lambda_{j}\left(t_{1}+1\right)}-1\right) \frac{\prod_{l=1}^{t_{1}-1} \lambda_{k}(l)}{\prod_{l=1}^{t_{1}} \lambda_{j}(l)} .
\end{aligned}
$$

Similar to the estimation in (4.3.17), we can verify that

$$
\left|\sum_{t_{1}=t}^{\infty}\left[\cos \left(t_{1}+\frac{1}{2}\right)\right] \cdot\left(t_{1}+1\right)^{\underline{\delta}}\right| \leq M_{1}(t+1)^{\underline{\delta}}
$$


By (4.3.23) and (4.3.24), we can get

$$
\begin{aligned}
& \left|\sum_{t_{1}=t}^{\infty}\left[\cos \left(t_{1}+\frac{1}{2}\right)\right] \cdot\left(t_{1}+1\right)^{\underline{\delta}}\left(\frac{\lambda_{k}\left(t_{1}\right)}{\lambda_{j}\left(t_{1}+1\right)}-1\right) \frac{\prod_{l=1}^{t_{1}-1} \lambda_{k}(l)}{\prod_{l=1}^{t_{1}} \lambda_{j}(l)}\right| \\
\leq & M_{1}(t+1)^{\underline{\delta}}\left|\left(\frac{\lambda_{k}(t)}{\lambda_{j}(t+1)}-1\right) \frac{\prod_{l=1}^{t-1} \lambda_{k}(l)}{\prod_{l=1}^{t} \lambda_{j}(l)}\right| \\
& +\sum_{t_{1}=t}^{\infty}\left\{M_{1}\left(t_{1}+2\right)^{\underline{\delta}}\left|\triangle\left(\frac{\lambda_{k}\left(t_{1}\right)}{\lambda_{j}\left(t_{1}+1\right)}-1\right)\right| \cdot\left|\frac{\prod_{l=1}^{t_{1}-1} \lambda_{k}(l)}{\prod_{l=1}^{t_{1}} \lambda_{j}(l)}\right|\right\} \\
& +\sum_{t_{1}=t}^{\infty}\left\{M_{1}\left(t_{1}+2\right)^{\underline{\delta}}\left|\left(\frac{\lambda_{k}\left(t_{1}+1\right)}{\lambda_{j}\left(t_{1}+2\right)}-1\right)\left(\frac{\lambda_{k}\left(t_{1}\right)}{\lambda_{j}\left(t_{1}+1\right)}-1\right)\right| \cdot\left|\frac{\prod_{l=1}^{t_{1}-1} \lambda_{k}(l)}{\prod_{l=1}^{t_{1}} \lambda_{j}(l)}\right|\right\} \\
\leq & \left.\left.M_{1}(t+1)^{\underline{\delta}}\left|\left(\frac{\lambda_{k}(t)}{\lambda_{j}(t+1)}-1\right) \frac{\prod_{l=1}^{t-1} \lambda_{k}(l)}{\prod_{l=1}^{t} \lambda_{j}(l)}\right| \mid \frac{\lambda_{k}\left(t_{1}\right)}{\lambda_{j}\left(t_{1}+1\right)}-1\right)|\cdot| \frac{\prod_{l=1}^{t_{1}-1} \lambda_{k}(l)}{\prod_{l=1}^{t_{1}} \lambda_{j}(l)} \mid\right\} \\
& +M_{1}(t+2)^{\underline{\delta}} \sum_{t_{1}=t}^{\infty}\left\{\left|\triangle\left(\frac{\lambda_{k}\left(t_{1}\right)}{\lambda_{j}\left(t_{1}+1\right)}-1\right)\right| \cdot\left|\frac{\prod_{l=1}^{t_{1}-1} \lambda_{k}(l)}{\prod_{l=1}^{t_{1}} \lambda_{j}(l)}\right|\right\} \\
& +M_{1}(t+2)^{\underline{\delta}} \sum_{t_{1}=t}^{\infty}\left\{\mid\left(t_{1}+2\right)\right. \\
(4.3 .25) \leq & M_{3} t^{\underline{\delta}},
\end{aligned}
$$

for some $M_{3}>0$.

For $F_{2}$ in (4.3.21), using the identity (4.3.3) we have

$$
\begin{aligned}
\left|F_{2}\right| & \left.=\mid \sum_{t_{1}=t}^{\infty} \frac{\delta}{4\left(\sin \frac{1}{2}\right)^{2}}\left[\sin \left(t_{1}+1\right)\right] \cdot\left(t_{1}+1\right) \frac{\delta-1}{\lambda_{j}\left(t_{1}+1\right)}-1\right) \frac{\lambda_{k}\left(t_{1}\right)}{\prod_{l=1}^{t_{1}-1} \lambda_{k}(l)} \mid \\
& \leq \frac{|\delta|}{4\left(\sin \frac{1}{2}\right)^{2}} \sum_{t_{1}=t}^{\infty}\left|t_{j}(l)\right| \\
& \leq \frac{\delta-1}{1}|\cdot|\left(\frac{\lambda_{k}\left(t_{1}\right)}{\lambda_{j}\left(t_{1}+1\right)}-1\right) \frac{\prod_{l=1}^{t_{1}-1} \lambda_{k}(l)}{\prod_{l=1}^{t_{1}} \lambda_{j}(l)} \mid \\
(4.3 .26) & \leq M_{4} t^{-} t^{-}
\end{aligned}
$$

for some $M_{4}>0$. 
For $F_{3}$ in (4.3.21), similar to the estimation in (4.3.26) above, we have

$$
\begin{aligned}
& \left|\sum_{t_{1}=t}^{\infty} \frac{\delta}{4\left(\sin \frac{1}{2}\right)^{2}}\left(\sum_{t_{2}=t_{1}+1}^{\infty}\left[\sin \left(t_{2}+1\right)\right] \cdot(\delta-1) \frac{\delta-2}{2}\right)\left(\frac{\lambda_{k}\left(t_{1}\right)}{\lambda_{j}\left(t_{1}+1\right)}-1\right) \frac{\prod_{l=1}^{t_{1}-1} \lambda_{k}(l)}{\prod_{l=1}^{t_{1}} \lambda_{j}(l)}\right| \\
\leq & \frac{|\delta|}{4\left(\sin \frac{1}{2}\right)^{2}} \sum_{t_{1}=t}^{\infty}\left|\left(\sum_{t_{2}=t_{1}+1}^{\infty} \mid(\delta-1) t_{2} \frac{\delta-2}{2}\right)\left(\frac{\lambda_{k}\left(t_{1}\right)}{\lambda_{j}\left(t_{1}+1\right)}-1\right) \frac{\prod_{l=1}^{t_{1}-1} \lambda_{k}(l)}{\prod_{l=1}^{t_{1}} \lambda_{j}(l)}\right| \\
= & \frac{|\delta|}{4\left(\sin \frac{1}{2}\right)^{2}} \sum_{t_{1}=t}^{\infty}\left|\left(t_{1}+1\right) \frac{\delta-1}{\prod_{j}}\left(\frac{\lambda_{k}\left(t_{1}\right)}{\lambda_{j}\left(t_{1}+1\right)}-1\right) \frac{\prod_{l=1}^{t_{1}-1} \lambda_{k}(l)}{\prod_{l=1}^{t_{1}} \lambda_{j}(l)}\right| \\
(4.3 .27) & \leq M_{4} t^{\delta} .
\end{aligned}
$$

$>$ From (4.3.21), (4.3.25), (4.3.26) and (4.3.27), we get that

$$
\left|A_{2}\right| \leq M_{5} t^{\underline{\delta}}
$$

where $M_{5}=\frac{1}{2 \sin \frac{1}{2}} M_{3}+2 M_{4}>0$ is a positive constant.

By (4.3.13), (4.3.19) and (4.3.28), we get the estimation of $\sum_{t_{1}=t}^{\infty} \widetilde{R}(t)$ as

$$
\left|\left(\sum_{t_{1}=t}^{\infty} \widetilde{R}\left(t_{1}\right)\right)_{j k}\right| \leq\left|c_{j k}\right|\left(M_{1} M_{2}+M_{5}\right) t^{\underline{\delta}}
$$

which leads to the conclusion that $\sum_{t_{1}=t}^{\infty} \widetilde{R}\left(t_{1}\right) \rightarrow 0$ as $t \rightarrow \infty$.

Now, consider $\left(\sum_{t_{1}=t+1}^{\infty} \widetilde{R}\left(t_{1}\right)\right) \widetilde{R}(t)$. In this example,

$$
\begin{aligned}
\left.\mid\left[\sum_{t_{1}=t+1}^{\infty} \widetilde{R}\left(t_{1}\right)\right) \widetilde{R}(t)\right]_{j k} \mid & =\left|\sum_{v=1}^{n}\left[\left(\sum_{t_{1}=t+1}^{\infty} \widetilde{r}_{j v}\left(t_{1}\right)\right) \widetilde{r}_{v k}(t)\right]\right| \\
& \leq \sum_{v=1}^{n}\left|\sum_{t_{1}=t+1}^{\infty} \widetilde{r}_{j v}\left(t_{1}\right)\right| \cdot\left|\widetilde{r}_{v k}(t)\right| \\
& \leq \sum_{v=1}^{n}\left|c_{j v}\right|\left(M_{1} M_{2}+M_{5}\right) t^{\underline{\delta}} \cdot\left|\left(c_{v k} \sin t \cdot t^{\underline{\delta}}\right) \frac{\prod_{l=1}^{t-1} \lambda_{k}(l)}{\prod_{l=1}^{t} \lambda_{v}(l)}\right| \\
& \leq n \cdot c^{2}\left(M_{1} M_{2}+M_{5}\right) M_{2}\left(t^{\underline{\delta}} \cdot t^{\underline{\delta}}\right),
\end{aligned}
$$

where $c=\max \left\{\left|c_{j k}\right|\right\}$.

Since $\delta<-\frac{1}{2}$, we have that $\left(\sum_{t_{1}=t+1}^{\infty} \widetilde{R}\left(t_{1}\right)\right) \widetilde{R}(t) \in l^{1}[1, \infty)$. Therefore, the system (4.3.9) is right almost diagonal.

We can obtain stronger results if we narrow our discussion to the case $n=2$.

EXAmple 4.3.2. Consider a difference system on the interval $[1, \infty)$,

$$
Y(t+1)=\left(\left[\begin{array}{cc}
\lambda_{1}(t) & 0 \\
0 & \lambda_{2}(t)
\end{array}\right]+\left[\begin{array}{cc}
0 & c_{12}(\sin t) \cdot t \underline{\delta_{1}} \\
c_{21}(\sin t) \cdot t \underline{\delta_{2}} & 0
\end{array}\right]\right) Y(t) .
$$

Let, 
(1) the system is potentially oscillatory and $\left|\lambda_{j}(t)\right| \geq \mu>0$ for some positive $\mu$,

(2) $\delta_{j}<0,\left|\frac{\lambda_{k}(t)}{\lambda_{j}(t+1)}-1\right|<M, \frac{1}{t-\delta_{j}+1}\left(\frac{\lambda_{k}(t)}{\lambda_{j}(t+1)}-1\right) \in l^{1}[1, \infty), \triangle\left(\frac{\lambda_{k}(t)}{\lambda_{j}(t+1)}-1\right) \in l^{1}[1, \infty)$,

$\left(\frac{\lambda_{k}(t+1)}{\lambda_{j}(t+2)}-1\right)\left(\frac{\lambda_{k}(t)}{\lambda_{j}(t+1)}-1\right) \in l^{1}[1, \infty)$ for $j, k=1,2$, and $j \neq k$,

(3) $\delta_{1}+\delta_{2}<-1$.

Then (4.3.31) is right almost diagonal.

PRoOF. In this example,

$$
\widetilde{R}(t)=\left(\left(c_{j k}(\sin t) \cdot t^{\delta_{j}}\right) \frac{\prod_{l=1}^{t-1} \lambda_{k}(l)}{\prod_{l=1}^{t} \lambda_{j}(l)}\right)_{j, k=1}^{n} .
$$

Similar to the analysis of Example 4.3.1, conditions (1) and (2) of this example lead to that

$$
\left|\left(\sum_{t_{1}=t}^{\infty} \widetilde{R}\left(t_{1}\right)\right)_{j k}\right| \leq M_{6} t^{\delta_{j}}
$$

for some $M_{6}>0$. Hence, we conclude that $\sum_{t_{1}=t}^{\infty} \widetilde{R}\left(t_{1}\right) \rightarrow 0$ as $t \rightarrow \infty$.

At the same time, notice that

$$
\begin{aligned}
\left|\left(\sum_{t_{1}=t+1}^{\infty} \widetilde{R}\left(t_{1}\right)\right) \widetilde{R}(t)\right| & =\left[\begin{array}{cc}
\left|\left(\sum_{t_{1}=t+1}^{\infty} \widetilde{r}_{12}\left(t_{1}\right)\right) \widetilde{r}_{21}(t)\right| & 0 \\
0 & \left|\left(\sum_{t_{1}=t+1}^{\infty} \widetilde{r}_{21}\left(t_{1}\right)\right) \widetilde{r}_{12}(t)\right|
\end{array}\right] \\
& \leq\left[\begin{array}{cc}
M_{6} t \underline{\delta_{1}} \cdot M_{2}\left|c_{21}(\sin t) \cdot t \underline{\delta_{2}}\right| & 0 \\
0 & M_{6} t \underline{\delta_{2}} \cdot M_{2}\left|c_{12}(\sin t) \cdot t^{\underline{\delta_{1}}}\right|
\end{array}\right] \\
& \leq M_{7}\left[\begin{array}{cc}
t \underline{\delta_{1}} \cdot t \underline{\delta_{2}} & 0 \\
0 & t \underline{\delta_{2}} \cdot t \underline{\delta_{1}}
\end{array}\right],
\end{aligned}
$$

for some $M_{7}>0$.

Since $t^{\delta_{j}} \sim t^{\delta_{j}}$ and $\delta_{1}+\delta_{2}<-1$, we get that $\left(\sum_{t_{1}=t+1}^{\infty} \widetilde{R}\left(t_{1}\right)\right) \widetilde{R}(t) \in l^{1}[1, \infty)$. Therefore, the system (4.3.31) is right almost diagonal.

It may be possible to extend the analysis in here to the more general and interesting setting of time scales, see e.g. [5]. However, we cannot see how to give such concrete examples as the Example 4.3.1, 4.3.2 in the setting of time scales.

Theorem 4.2.6 is a more convenient tool, (than Theorem 4.2.4), to apply in order to show that a potentially oscillatory difference system is right almost diagonal. This is due to the conditions of Theorem 4.2.6 imposed directly on $R(t)$ rather than on $\widetilde{R}(t)$. We will demonstrate this in the following example.

ExAmple 4.3.3. Consider a difference system on the interval $[1, \infty)$,

$$
Y(t+1)=\left(\left[\begin{array}{cc}
1+\frac{1}{t} & 0 \\
0 & 1+\frac{1}{t+1}
\end{array}\right]+\left[\begin{array}{cc}
0 & c_{12} \\
c_{21} & 0
\end{array}\right] \frac{\sin t}{t}\right) Y(t) .
$$

We show that it is a right almost diagonal difference system. 
PROOF. In this example, we can see that for any $1 \leq t_{1}<t_{2} \leq \infty$,

$$
\prod_{l=t_{1}}^{t_{2}} \frac{\lambda_{1}(l)}{\lambda_{2}(l)}=\prod_{l=t_{1}}^{t_{2}} \frac{1+\frac{1}{l}}{1+\frac{1}{l+1}}=\frac{\prod_{l=t_{1}}^{t_{2}} \frac{l+1}{l}}{\prod_{l=t_{1}}^{t_{2}} \frac{l+2}{l+1}}=\frac{\frac{t_{2}+1}{t_{1}}}{\frac{t_{2}+2}{t_{1}+1}}=\left(1+\frac{1}{t_{1}}\right)\left(1-\frac{1}{t_{2}+2}\right)
$$

which leads to $\frac{1}{2}<\left|\prod_{l=t_{1}}^{t_{2}} \frac{\lambda_{1}(l)}{\lambda_{2}(l)}\right|<2$. Therefore, (4.3.33) is a potentially oscillatory difference system. Now we want to apply Theorem 4.2.6 to show that (4.3.33) is right almost diagonal.

First, consider $\sum_{t_{1}=t}^{\infty} R\left(t_{1}\right)$. We will estimate $\sum_{t_{1}=t}^{\infty} \frac{\sin t_{1}}{t_{1}}$ as follows,

$$
\begin{aligned}
\sum_{t_{1}=t}^{\infty} \frac{\sin t_{1}}{t_{1}} & =\left[-\frac{\cos \left(t_{1}-1 / 2\right)}{2 \sin \frac{1}{2}} \cdot \frac{1}{t_{1}}\right]_{t}^{\infty}+\sum_{t_{1}=t}^{\infty} \frac{\cos \left(t_{1}+1 / 2\right)}{2 \sin \frac{1}{2}} \triangle \frac{1}{t_{1}} \\
& =\frac{\cos (t-1 / 2)}{2 \sin \frac{1}{2}} \cdot \frac{1}{t}-\sum_{t_{1}=t}^{\infty} \frac{\cos \left(t_{1}+1 / 2\right)}{2 \sin \frac{1}{2}} \frac{1}{t_{1}\left(t_{1}+1\right)} .
\end{aligned}
$$

Therefore, we get

$$
\left|\sum_{t_{1}=t}^{\infty} \frac{\sin t}{t}\right| \leq\left|\frac{1}{2} \cdot \frac{1}{t}\right|+\frac{1}{2} \sum_{t_{1}=t}^{\infty} \frac{1}{t_{1}\left(t_{1}+1\right)} \leq \frac{1}{t}
$$

and then

$$
\left\|\sum_{t_{1}=t}^{\infty} R\left(t_{1}\right)\right\|=\left\|\sum_{t_{1}=t}^{\infty}\left[\begin{array}{cc}
0 & c_{12} \\
c_{21} & 0
\end{array}\right] \frac{\sin t_{1}}{t_{1}}\right\| \leq \frac{2 C}{t},
$$

where $C=\max \left\{\left|c_{12}\right|,\left|c_{21}\right|\right\}$. It is obvious that $\sum_{t_{1}=t}^{\infty} R\left(t_{1}\right) \rightarrow 0$ as $t \rightarrow \infty$.

Second, we see that

$$
D^{-1}(t)=\left[\begin{array}{cc}
\frac{1}{1+\frac{1}{t}} & 0 \\
0 & \frac{1}{1+\frac{1}{t+1}}
\end{array}\right]
$$

which implies that $\left\|D^{-1}(t)\right\|<2$ on $[1, \infty)$. We now obtain

$$
\begin{aligned}
& \left\|D^{-1}(t)\left[(I-D(t))\left(\sum_{t_{1}=t}^{\infty} R\left(t_{1}\right)\right)+\left(\sum_{t_{2}=t+1}^{\infty} R\left(t_{2}\right)\right)(D(t)-I)\right]\right\| \\
\leq & 2\left(\left\|\left[\begin{array}{cc}
-\frac{1}{t} & 0 \\
0 & -\frac{1}{t+1}
\end{array}\right]\right\| \cdot\left\|\sum_{t_{1}=t}^{\infty} R\left(t_{1}\right)\right\|+\left\|\sum_{t_{2}=t+1}^{\infty} R\left(t_{2}\right)\right\| \cdot\left\|\left[\begin{array}{cc}
\frac{1}{t} & 0 \\
0 & \frac{1}{t+1}
\end{array}\right]\right\|\right) \\
\leq & 2\left(\left|\frac{1}{t}+\frac{1}{t+1}\right| \cdot \frac{2 C}{t}+\frac{2 C}{t+1} \cdot\left|\frac{1}{t}+\frac{1}{t+1}\right|\right) \\
\leq & \frac{16 C}{t^{2}}
\end{aligned}
$$

Therefore,

$$
D^{-1}(t)\left[(I-D(t))\left(\sum_{t_{1}=t}^{\infty} R\left(t_{1}\right)\right)+\left(\sum_{t_{2}=t+1}^{\infty} R\left(t_{2}\right)\right)(D(t)-I)\right] \in l^{1}[1, \infty) .
$$


Third,

$$
\left\|D^{-1}(t)\left(\sum_{t_{1}=t+1}^{\infty} R\left(t_{1}\right)\right) R(t)\right\| \leq 2 \cdot \frac{2 C}{t+1} \cdot 2 C\left|\frac{\sin t}{t}\right| \leq \frac{8 C^{2}}{t(t+1)},
$$

which implies that $D^{-1}(t)\left(\sum_{t_{1}=t+1}^{\infty} R\left(t_{1}\right)\right) R(t) \in l^{1}[1, \infty)$.

Fourth, since (4.3.33) is potentially oscillatory and $\left\|D^{-1}(t)\right\|<2$, there exists some $M_{8}>0$ such that

$$
\begin{aligned}
\| & \| \sum_{t_{1}=t+1}^{\infty}\left[\Phi ^ { - 1 } ( t _ { 1 } ) D ^ { - 1 } ( t _ { 1 } ) \left(\left(I-D\left(t_{1}\right)\right) \sum_{t_{2}=t_{1}}^{\infty} R\left(t_{2}\right)\right.\right. \\
& \left.\left.\left.+\sum_{t_{3}=t_{1}+1}^{\infty} R\left(t_{3}\right)\left(D\left(t_{1}\right)-I\right)\right) \Phi\left(t_{1}\right)\right]\right\} \cdot \Phi^{-1}(t+1) R(t) \Phi(t) \| \\
\leq & M_{8} \sum_{t_{1}=t+1}^{\infty}\left\|D^{-1}\left(t_{1}\right)\left(\left(I-D\left(t_{1}\right)\right) \sum_{t_{2}=t_{1}}^{\infty} R\left(t_{2}\right)+\sum_{t_{3}=t_{1}+1}^{\infty} R\left(t_{3}\right)\left(D\left(t_{1}\right)-I\right)\right)\right\| \cdot\|R(t)\| \\
\leq & M_{8}\left(\sum_{t_{1}=t+1}^{\infty} \frac{16 C}{t_{1}^{2}}\right) \cdot 2 C\left|\frac{\sin t}{t}\right| \leq \frac{32 C^{2} M_{8}}{t} \cdot \sum_{t_{1}=t+1}^{\infty} \frac{1}{t_{1}^{2}} \leq \frac{32 C^{2} M_{8}}{t} \cdot \sum_{t_{1}=t+1}^{\infty} \frac{1}{t_{1}\left(t_{1}-1\right)} \\
= & \frac{32 C^{2} M_{8}}{t^{2}}
\end{aligned}
$$

Hence, the condition (4.2.44) also holds in this example. We conclude that (4.3.33) is right almost diagonal.

In all the examples of this paper, $\left|\widetilde{R}\left(t_{1}\right)\right| \notin l^{1}[1, \infty)$, therefore, Proposition 2.2 in [16] and Lemma 2.1 in [3] do not apply. 


\section{Bibliography}

[1] J. E. Avron and A. Elgart, Adiabatic theorem without a gap condition, Comm. Math. Phys. 203 (1999) 445-463.

[2] H. Behncke and C. Remling, Asymptotic integration of linear differential equations, J. Math. Anal. Appl. 210 (1997) 585-597.

[3] Z. Benzaid, D.A. Lutz, Asymptotic representation of solutions of perturbed systems of linear difference equations, Stud. Appl. Math. 77 (1987) 195-221.

[4] S. Bodine, D.A. Lutz, Asymptotic solutions and error estimates for linear systems of difference and differential equations, J. Math. Anal. Appl. 290 (2004) 343-362.

[5] S. Bodine, M. Bohner and D. A. Lutz, Asymptotic behavior of solutions of dynamic equations, Journal of Mathematical Sciences, Vol. 124 (2004) No. 4, 5110-5118,

[6] S. Bodine and R. J. Sacker, Asymptotic diagonalization of linear difference equations. In memory of W.A. Harris, Jr., J. Differ. Equations Appl. 7 (2001) 637-650,

[7] M. Bohner and A. Peterson, Dynamic equations on time scales. An introduction with applications, Birkhauser, Boston, 2001.

[8] C. V. Coffman, Asymptotic behavior of solutions of ordinary difference equations, Trans. Amer. Math. Soc. 110 (1964) 22-51.

[9] M. S. P. Eastham, The asymptotic solution of linear differential systems, University Press, Oxford, 1989.

[10] P. Ehrenfest, On adiabatic changes of a system in connection with the quantum theory, Proc. Royal Acad. Amsterdam, 19 (1917), 576-597.

[11] S. Elaydi, Asymptotics for linear difference equations I: basic theory, J. Differ. Equations Appl. 5 (1999) 563-589.

[12] U. Elias and H. Gingold, A method for asymptotic integration of almost diagonal systems, Asymptotic Analysis, 29 (2002) 343-357.

[13] U. Elias and H. Gingold, A framework for asymptotic integration of differential systems, Asymptotic Analysis, 35 (2003) 281-300.

[14] H. Gingold, Almost diagonal systems in asymptotic integration, Proceedings of the Edinburgh Mathematical Society, 28 (1985), 143-158.

[15] H. Gingold, In general, the less degeneracy the less transition. A principle for time dependent Hamiltonian systems in quantum mechanics, J. Math. Phys. 28 (1987),2400-2406.

[16] H. Gingold, Almost diagonal systems of linear difference equations. In memory of W. A. Harris, Jr., J. Differ. Equations Appl., 7 (2001) 685-700.

[17] H. Gingold and P.F. Hsieh, Global approximation of perturbed Hamiltonian differential equations with several turning points, SIAM J. Math. Anal., 18 (1987), 1275-1293.

[18] H. Gingold, J. She and W.E. Zorumski, Local principles of wave propagation in homogeneous media, J. Acoust. Soc. Amer. 93 (1993), 599-604.

[19] H. Gingold and F. Xue, A new technique for asymptotic integration of potentially oscillatory differential systems, WVU preprint, 2005.

[20] H. Gingold and F. Xue, On Asymptotic Summation of Potentially Oscillatory Difference Systems, WVU, preprint, 2005.

[21] H. Gingold and F. Xue, On the Asymptotic Summation of Difference Systems, WVU, preprint, 2005.

[22] W. A. Harris Jr. and D. Lutz, On the asymptotic integration of linear differential systems, J. Math. Anal. Appl., 48 (1974) 1-16.

[23] W. A. Harris Jr. and D. Lutz, A unified theory of asymptotic integration, J. Math. Anal. Appl., 57 (1977) 571-586. 
[24] P. Hartman and A. Wintner, Asymptotic integration of linear differential equations, Amer. J. Math. 77 (1955), 45-86, 932.

[25] P.F. Hsieh and Y. Sibuya, Basic Theory of Ordinary Differential Equations, Springer, (1999).

[26] P. Hsieh and F. Xie, Asymptotic diagonalization of a system of linear ordinary differential equations, Dynam. Contin. Discrete Impuls. Systems, 1 (1996), 51-74.

[27] P. F. Hsieh, F. Xie, On asymptotic diagonalization of linear ordinary differential equations, Dynam. Contin. Discrete Impuls. Systems, 4 (1998) 351-377.

[28] W.G. Kelley and A.C. Peterson, Difference equations. An introduction with applications, Second edition, Academic Press, 2001.

[29] N. Levinson, The asymptotic nature of solutions of linear differential equations, Duke Math. J., 15 (1948), 111-126.

[30] Z.-H. Li, The asymptotic estimates of solutions of difference equations, J. Math. Anal. Appl. 94 (1983) 181-192.

[31] R.L. Liboff, Introductory Quantum Mechanics, Holden-Day, San Francisco, (1980).

[32] R. Medina and M. Pinto, Linear differential systems with conditionally integrable coefficients, J. Math. Anal. Appl., 166 (1992) 52-64.

[33] F. Olver, Asymptotics and special functions. Computer science and applied mathematics. Academic Press, 1974.

[34] W. R. Wasow, Asymptotic expansions for ordinary differential equations, John Wiley, New York, (1965). 